UNIVERSITE DU QUEBEC

MEMOIRE PRESENTE A

L'UNIVERSITE DU QUEBEC EN ABITIBI-TEMISCAMINGUE

COMME EXIGENCE PARTIELLE DE

LA MAITRISE EN GESTION DES P.M.O.

PAR

GINETTE PAUL

PROBLEMATIQUE DE LA PETITE ENTREPRISE EN PHASE DE DEMARRAGE FOCALISEE SUR LA NECESSITE D'UN MANAGEMENT STRATEGIQUE PERFORMANT

Le cas d'une petite entreprise de l'industrie de l'audiovisuel: Imagem

NOVEMBRE 1988 


\section{Bibliothèque}

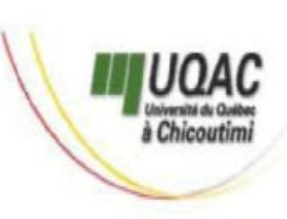

\section{Mise en garde/Advice}

Afin de rendre accessible au plus Motivated by a desire to make the grand nombre le résultat des results of its graduate students' travaux de recherche menés par ses research accessible to all, and in étudiants gradués et dans l'esprit des accordance with the rules règles qui régissent le dépôt et la governing the acceptation and diffusion des mémoires et thèses diffusion of dissertations and produits dans cette Institution, theses in this Institution, the I'Université du Québec à Université du Québec à Chicoutimi (UQAC) est fière de Chicoutimi (UQAC) is proud to rendre accessible une version make a complete version of this complète et gratuite de cette œuvre. work available at no cost to the reader.

L'auteur conserve néanmoins la The author retains ownership of the propriété du droit d'auteur qui copyright of this dissertation or protège ce mémoire ou cette thèse. thesis. Neither the dissertation or Ni le mémoire ou la thèse ni des thesis, nor substantial extracts from extraits substantiels de ceux-ci ne it, may be printed or otherwise peuvent être imprimés ou autrement reproduced without the author's reproduits sans son autorisation. permission. 


\section{Novembre 1988}

Résumé d'un mémoire présenté à l'université du québec en Abitibi-Témiscamingue (UQAT), dans le cadre de la maitrise en Gestion des petites et moyennes organisations (MGPMO).

Problématique de la petite entreprise en phase de démarrage focalisée sur la nécessité d'un management stratégique performant;

Lecas d'une petite entreprise de l'industrie de l'audiovisue l: Imagem.

Notre mémoire aborde la problématique des petites entreprises en phase de démarrage, sous l'angle du management stratégique. Notre étude s'inscrit dans le cadre d'une recherche-action et s'inspire de la méthodologie des systèmes souples de peter B. Checkland. Ainsi, nous considérons que la méthodologie des systèmes souples de checkland, nous a bien servis dans notre recherche. Premierement, elle permettait de laisser une grande place aux personnes impliqués dans le diagnostic et elle facilitait le consensus. Deuxièmement, son impact s'est avéré très positif pour améliorer la santé globale de l'entreprise et pour assurer le développement de l'autonomie des propriétaires à gérer leur entreprise de façon plus efficace et globale.

Notre quête des facteurs qui permettent d'éclairer la problématique de ces petites entreprises, s'appuie sur l'hypothèse que: les marchés en croissance compliquent la tâche quotidienne de leurs gestionnaires car ils les obligent à réévaluer régulièrement leur stratégie pour tenir compte d'un. environnement turbulent et de leurs ressources limitées. cette hypothèse a été vérifiée en majeure partie au cours du diagnostic organisationnel que nous avons réalisé auprès d'une jeune entreprise outaouaise de conception et de production audiovisuelle, Imagem.

Dans I'ensemble, notre recherche nous permet de croire que la problématique des petites entreprises en phase de démarrage, se situe d'abord au niveau d'une "quasi inexistence" de ressources humaines compétentes en gestion. Cette réalité se traduit entre autres: par une méconnaissance des principes de base du management; une forte concentration du temps du gestionnaire dans la prise de décisions de nature tactique et l'accomplissement d'activités quotidiennes, au détriment de décisions de nature stratégique et d'activités de planification; une difficulté pour le gestionnaire a analyser un problème dans son ensemble; etc.. De plus, nous constatons la faible marge de manoeuvre financière de la petite entreprise et de son ou ses propriétaires. 
Le cas de l'entreprise Imagem illustre bien l'ensemble de la problématique. D'une part, le diagnostic nous a permis d'identifier d'importantes difficultés aux niveaux des processus de planification, de production, d'allocation des ressources et de contrôle. D'autre part, il nous apparaissait évident que l'ensemble de ces difficultés présentait un lien étroit avec la "quasi inexistence" de ressources humaines compétentes en gestion et la faible marge de manoeuvre financière dont disposait l'entreprise et ses propriétaires pour procéder à des changements en profondeur. Il s'avéra toutefois que ces difficultés présentaient de bonnes chances d'être résolus par la mise sur pied d'un système complet de management stratégique, où on procéderait à un réalignement stratégique assorti de réajustements lors de la mise en oeuvre de la stratégie. Dans les faits, ce système a servi de base de discussion et d'apprentissage pour les propriétaires de l'entreprise. Le plan d'action, issu d'un processus de management stratégique complet et performant, priorisait nécessairement les décisions stratégiques qui permettaient d'améliorer les chances de survie de l'entreprise a court, moyen et long terme.

Finalement, l'importance que nous attachons aux besoins spécifiques à la petite entreprise en phase de démarrage, nous amène à proposer une redéfinition du rôle des nombreux intervenants en entrepreneurship. Les besoins de ce type d'entreprises se situent selon nous, au niveau des services techniques professionnels tels que: préparation d'études de marché et de faisabilité; conseils de base au niveau légal et fiscal; préparation d'un dossier de financement; etc.. Ces considérations entrainent donc la nécessité de procéder à une réaffectation des ressources humaines actuelles des intervenants en entrepreneurship vers des activités de soutien technique plutôt que promotionnelles. En outre, le style d'intervention de ces intervenants devra les rendre plus accessibles aux nouveaux entrepreneurs.

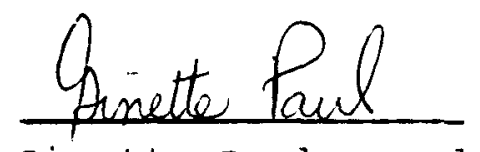

Ginette paul, candidate a la maitrise en Gestion des pMo

Gilles saint-pierre, directeur de recherche 


\begin{abstract}
Nous désirons par la présente remercier toutes les personnes qui ont participé à l'élaboration de ce dossier.
\end{abstract}

Messieurs Daniel Brazeau, Marc Gendron, Jean-Guy proulx
et le personnel de l'entreprise Imagem pour leur
collaboration.

Nos directeurs de mémoire et particulièrement Monsieur Gilles saint-pierre pour son appui et ses conseils judicieux.

Madame Marie Roy, pour sa disponibilité et son professionnalisme.

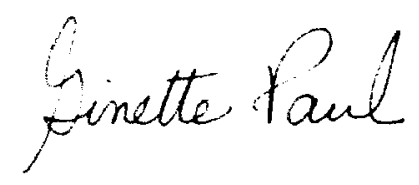

Ginette Paul 


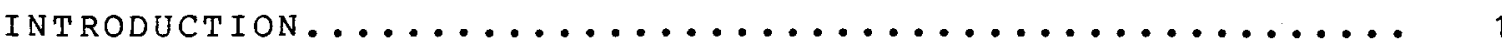

PARTIE A

I LA PROBLEMATIQUE DES PETITES ENTREPRISES EN PHASE 9 DE DEMARRAGE

PARTIE B

I I I'HISTORIQUE DE L'ORGANISATION................. 24

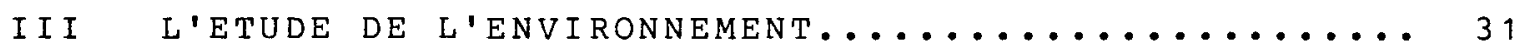

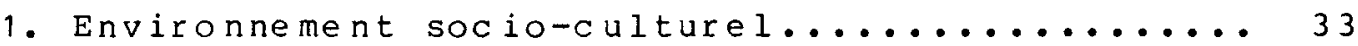

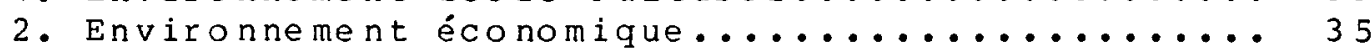

2.1 structure économique régionale.......... 35

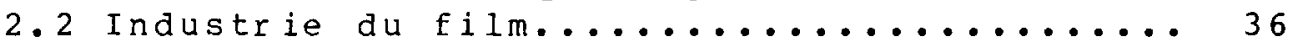

2.2. 1 I'industrie de façon générale..... 36

2.2.2 Les prospectives du marché de

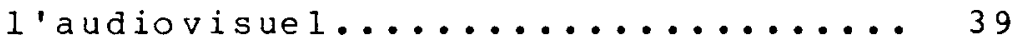

2.2.3 La problématique générale du marché

de 1 'audiovisue $1 \ldots . . . . . . . . . .441$

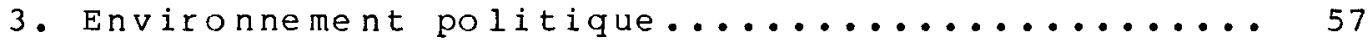

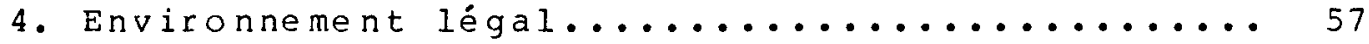

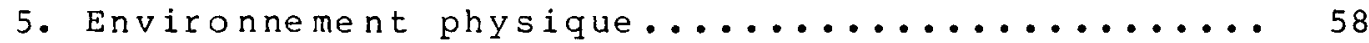

6. Perception d'Imagem dans 1'environnement..... 58

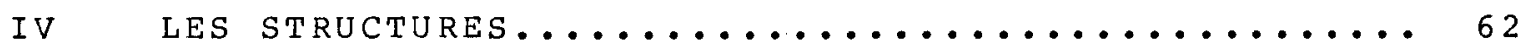

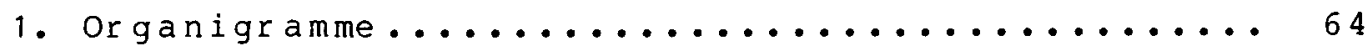

2. Responsabilités, description des tâches et

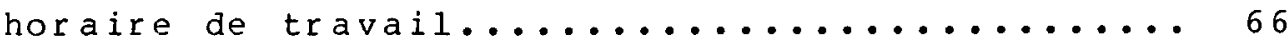
2. 1 Responsabilités et description de tâches... 66

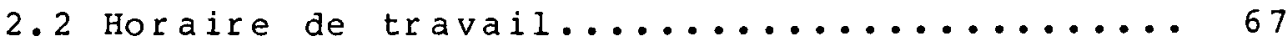

3. Compétences des ressources humaines..........668

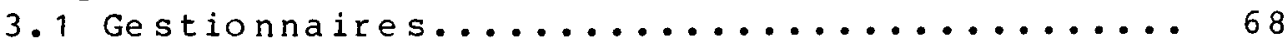
3. 2 Autres ressources humaines.............. 69

4. Politiques opérationnelles et réglements......770

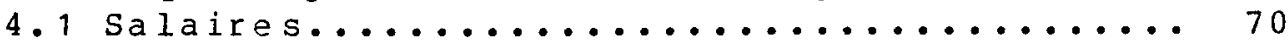

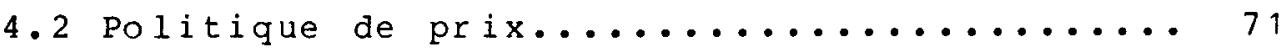

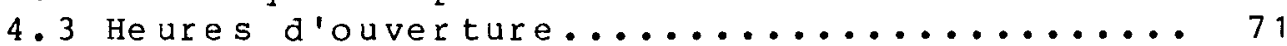
4.4 Autres é léments de 1 a structure.......... 72

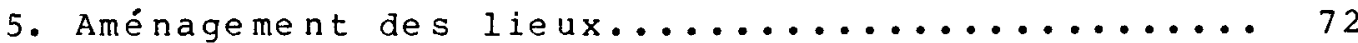


1. Environnement et super-système........... 77

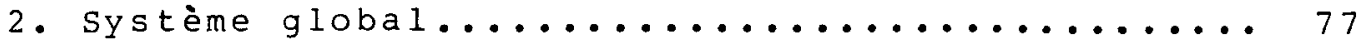

2.1 Sous-système planification stratégique.... 79

2.2 Sous-système développement............ 80

2.3 Sous-système promotion et vente.......... 82

2.4 sous-système ressources................ 83

2.5 sous-système production................... 84

2.6 Sous-système mise en marché............ 85

2.7 Sous-système contrôle............... 86

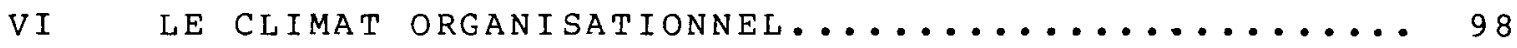

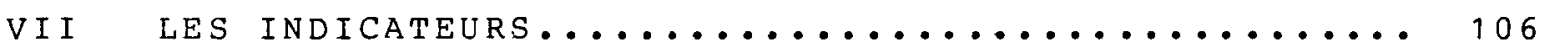

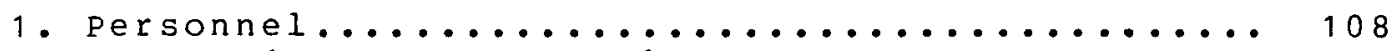

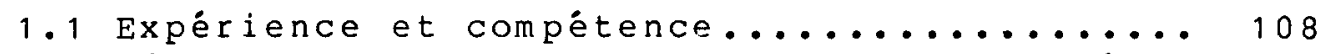

1.2 Répartition des tâches et responsabilités.. 110

1.3 Allocation des ressources humaines........ 110

1.4 Critères d'embauche et de sélection.......110

1.5 Négociation des contrats d'embauche....... 111

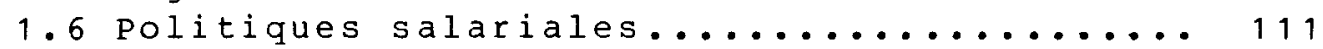

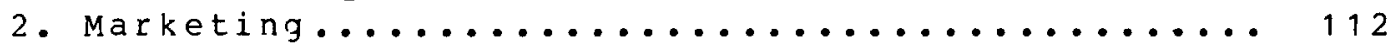

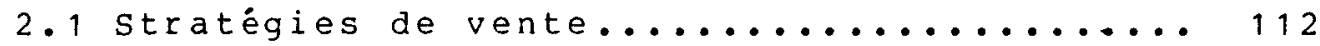

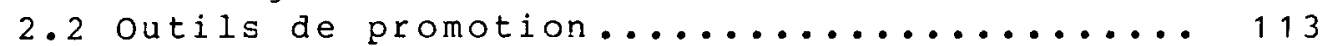

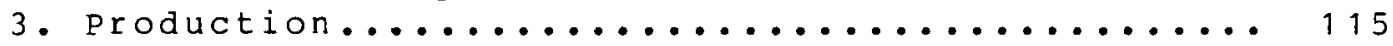

3. 1 Gestion des projets de production......... 115

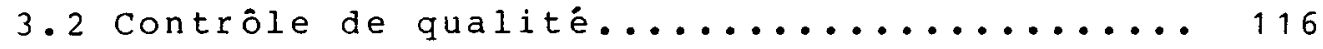

3 .3 Ressources techniques disponibles........ 117

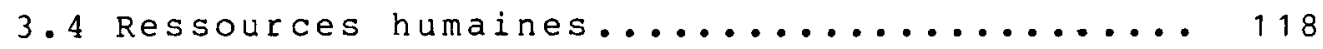

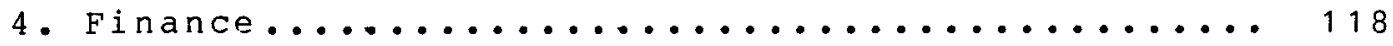

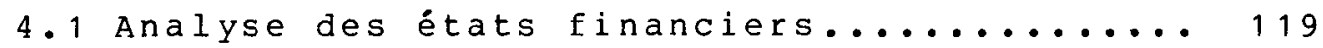

4. 2 Analyse du prix de revient des produits... 120

4.3 Calcul du seuil de rentabilité annuel..... 121

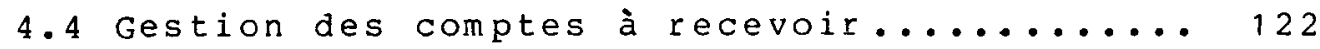

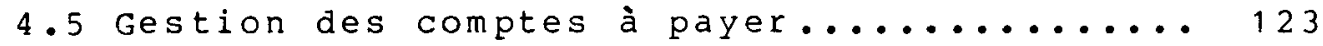

4.6 Autres exigibilités à court terme........ 124

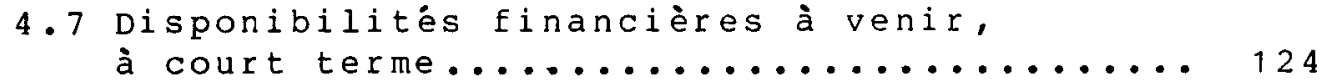

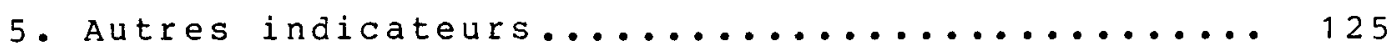

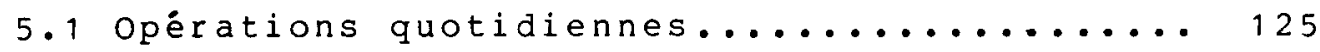

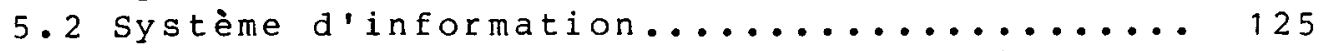

5.3 Respect de la mission et des objectifs

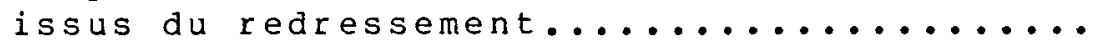


Vit li paroblematique de l'entreprise imagem......... 130

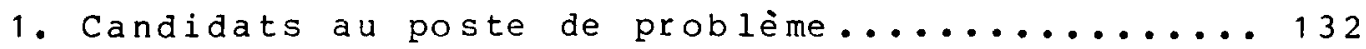

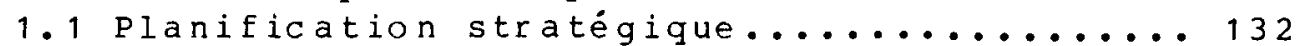

1.2 Dé veloppement de nouveaux produits....... 133

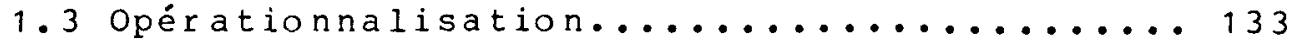

2. Choix et justification du point d'ancrage.... 135

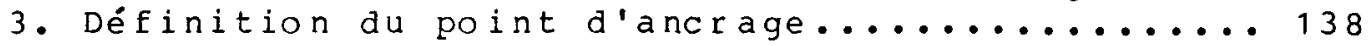

3.1 Le management stratégique............... 138

IX LA CONCEPTUALISATION ET LA COMPARAISON DU

SYSTEME PERTINENT........................ 142

1. Système de management stratégique........... 144

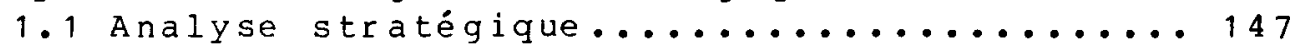

1.2 Dé veloppement du concept stratégique...... 148

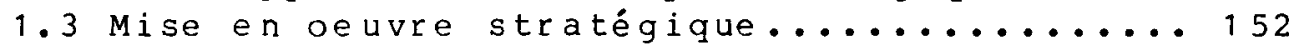

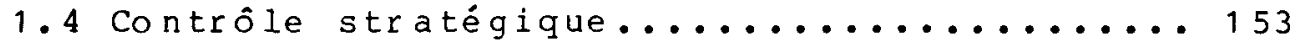

2. Sous-système d'information et de consultation.. 155

3. Validation du modèle conceptuel............ 158

4. Comparaison entre le modèle conceptuel et le

savoir-faire de l'entreprise à ce niveau...... 160

5. Actions correctrices à entreprendre.......... 162

$x$

L'OPERATIONNALISATION ET L'IMPLANTATION........ 164

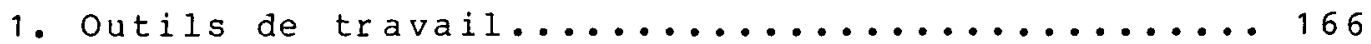

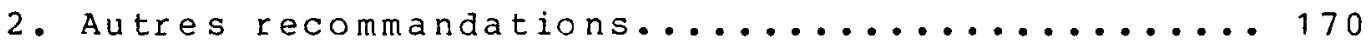

3. Opérationnalisation du système de

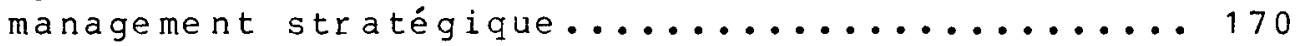

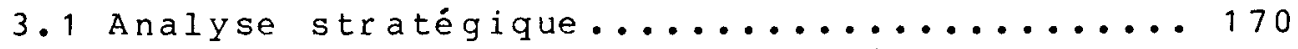

3. 2 Déve loppement du concept stratégique......174

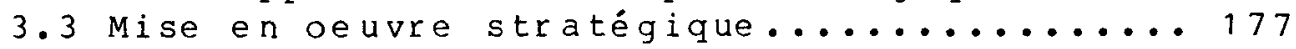

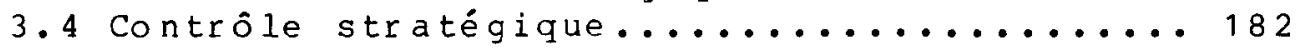

PARTIE C

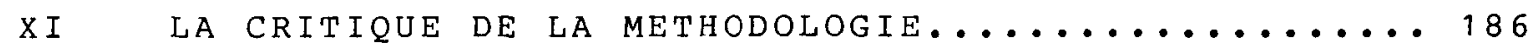

1. Etapes suggérées par la méthode............ 189

2. Diagnostic-intervention, une démarche

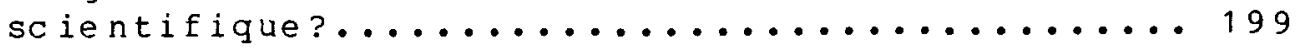

Xi I Limpact du diagnostic Chez IMAgem........... 203 
XII I UNE REFLEXION SUR L'INTERVENTION GOUVERNEMENTALE

AUPRES DES PETITES ENTREPRISES.................. 211

1. Besoins de la petite entreprise en phase de démarrage et portrait de son (ses)

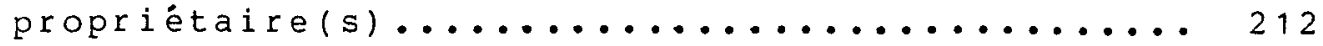

2. Rôle actuel des intervenants gouvernementaux

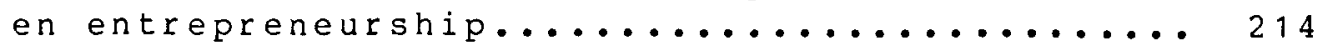

3. Rôle à privilégier chez les intervenants

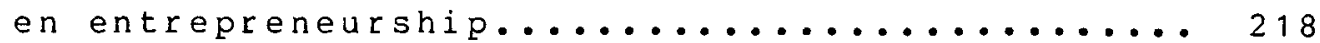

4. Un organisme idéal d'aide à l'entrepreneur.... 219

5. Pour une meilleure intervention des consultants gouvernementaux dans les

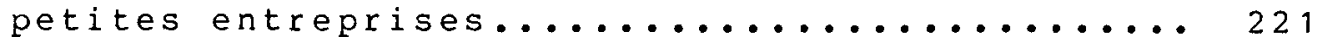

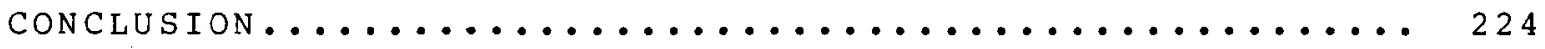




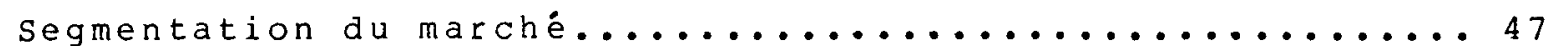

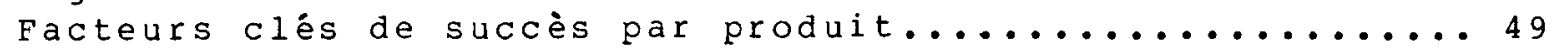

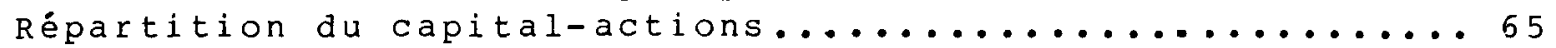

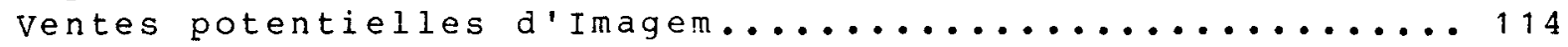

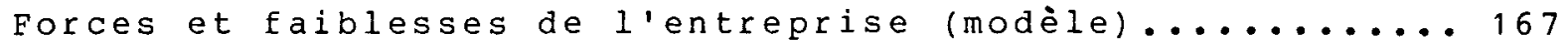

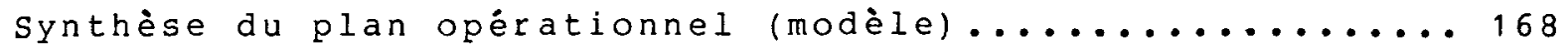
calendrier de mise en oeuvre stratégique (modèle)........ 169

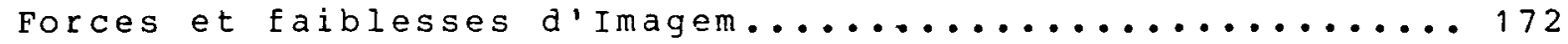

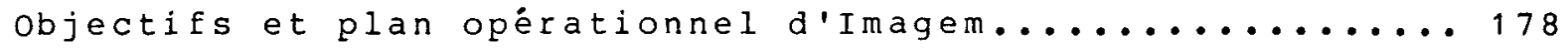




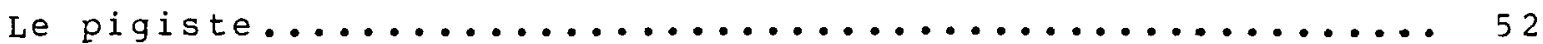

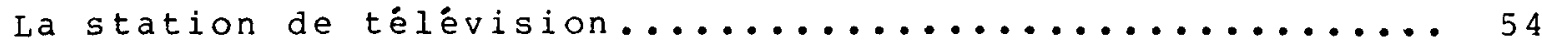

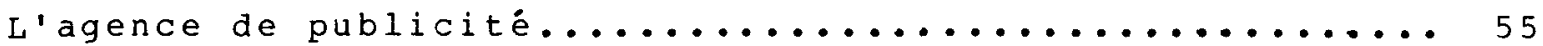

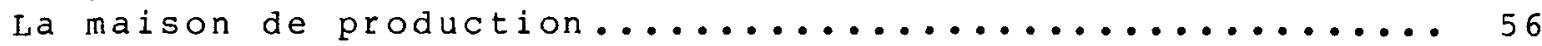

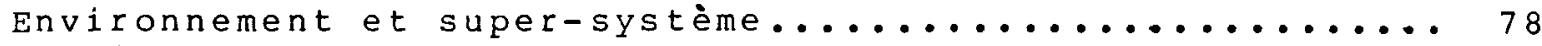

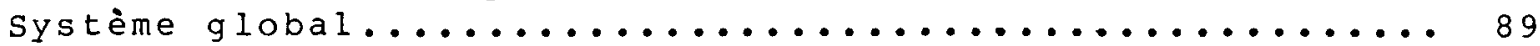

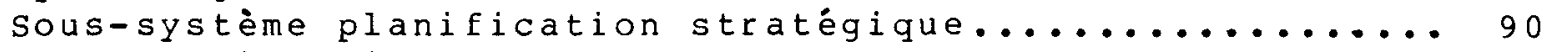

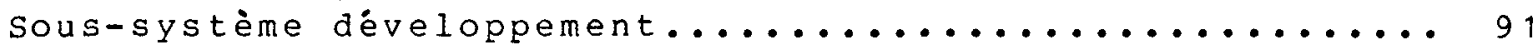

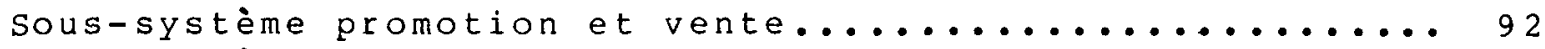

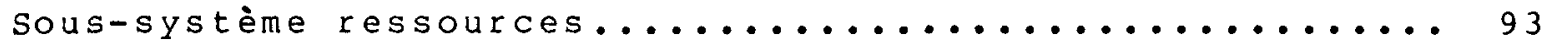

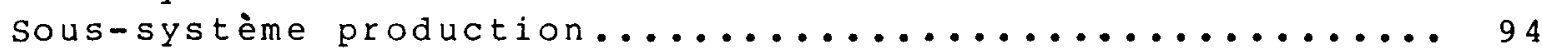

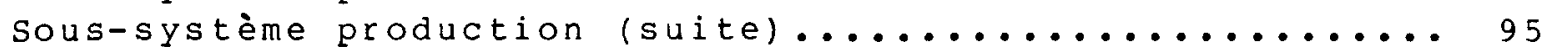

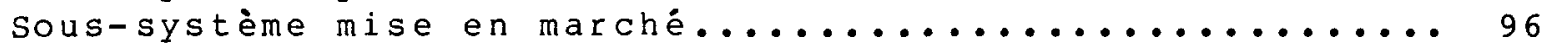

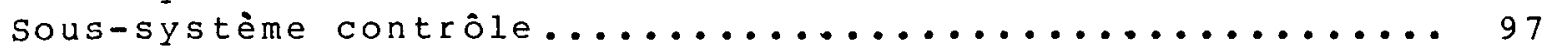

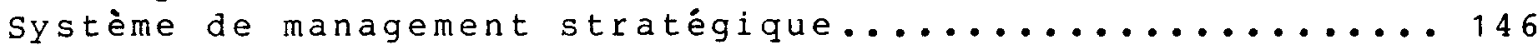

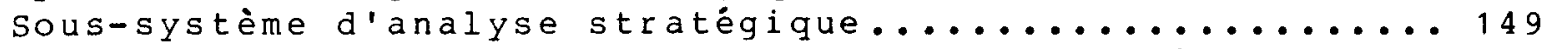

Sous-système de développement du concept stratégique.... 151

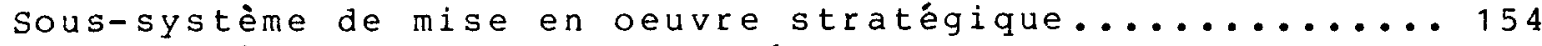

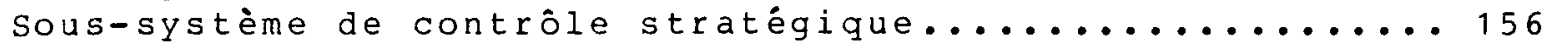

Sous-système d'information et de communication..........157

Méthodologie des systèmes souples de P.B. Checkland..... 188

Les intervenants en entrepreneurship de l'outaouas

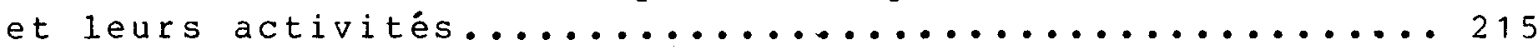




\section{LISTE DES ANNEXES}

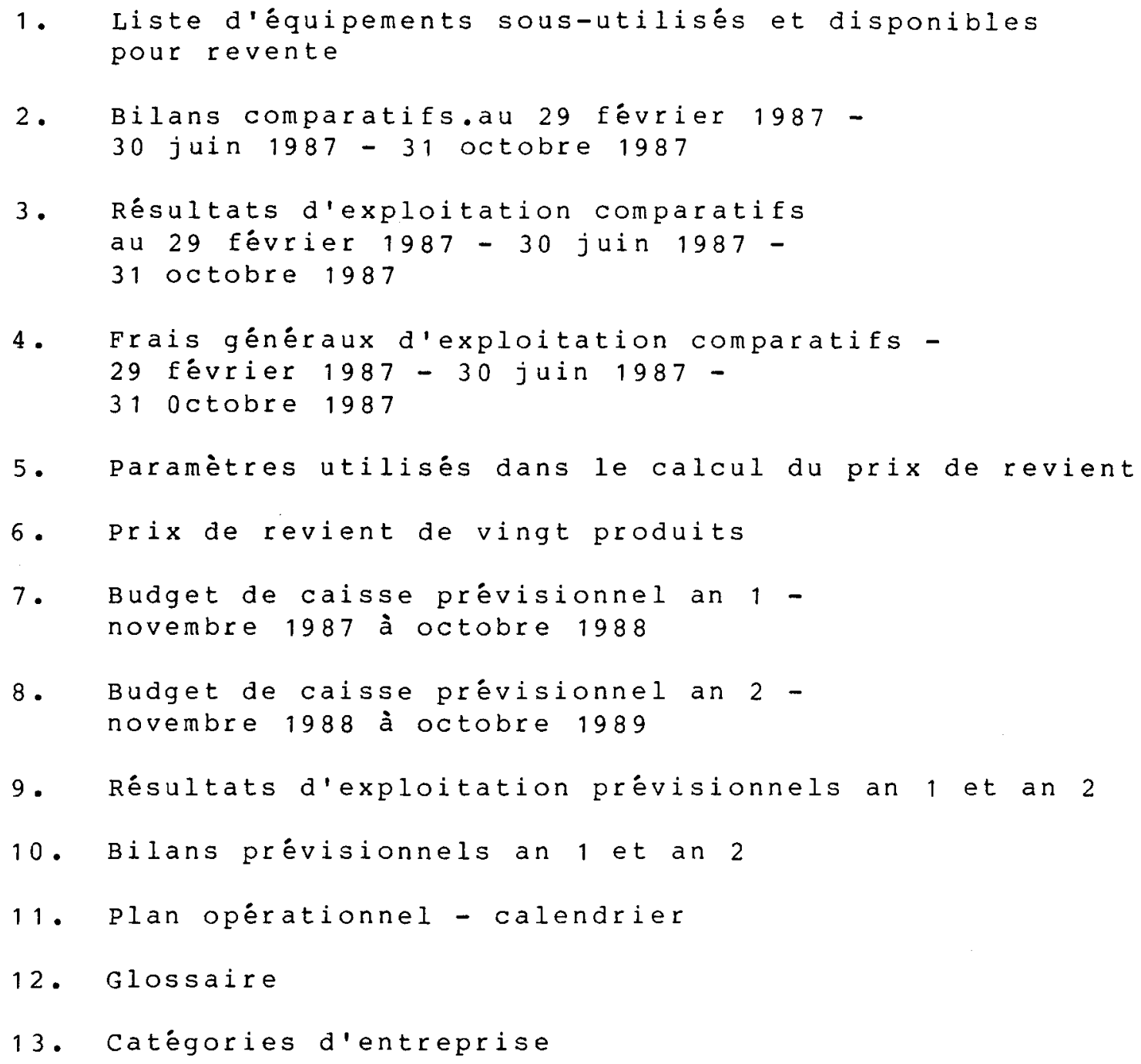




\section{LISTE DES SYMBOLES}

Identification d'un problème

$\varnothing$ 
INTRODUCTION 
Le présent mémoire s'inscrit dans le cadre du programme de maitrise en Gestion des petites et moyennes organisations (MGPMO), offert par l'université du québec en AbitibiTémiscamingue (UQAT) depuis 1983. Il constitue l'étape finale et déterminante du programme de formation.

S'il est vrai que ce mémoire respecte la philosophie et les orientations du programme d'étude, il est tout ausi vrai d'affirmer qu'il correspond à nos aspirations personnelles. D'une part, notre formation académique gravite depuis plusieurs années autour des différentes facettes de la gestion des petites et moyennes entreprises. D'autre part, notre expérience à titre de comptable, technicienne en vérification et consultante en démarrage et en diagnostic organisationnel dans les petites entreprises vient consolider la théorie acquise dans un premier temps. Ainsi, les expériences de démarrage dans une trentaine de projets d'entreprise nous ont permis d'acquérir une certaine connaissance des problèmes des jeunes entreprises et du portrait des entrepreneurs. Ces expériences constituent d'ailleurs le pivot de notre réflexion sur la problématique des petites entreprises en phase de démarrage de même que sur l'intervention gouvernementale à ce titre. Cette réflexion veut ausi donner le ton à l'ensemble de la recherche exposée dans notre mémoire. 
Nous définirons d'abord les concepts, petite entreprise et phase de demarrage, afin d'adopter un langage commun. puis nous nous interrogerons sur les éléments de la problématique des petites entreprises en phase de demarrage tels que: Quelles sont les caractéristiques de la petite entreprise en phase de démarrage? Comment son ou ses propriétaires contrôlent-ils l'entreprise? Quelle place le propriétaire accorde-t-il aux nombreuses facettes de la gestion de l'entreprise? En quoi la réussite de l'entreprise est-elle influencée par la personnalité du propriétaire? peut-on vraiment parler de problématique distinctive pour la petite entreprise en phase de démarrage? Autant de questions qui méritent une sérieuse réflexion de la part des personnes préoccupées par la réalité des petites entreprises.

Notre réflexion se poursuivra sur le rôle actuel des intervenants gouvernementaux dans la survie des petites entreprises et sur les améliorations souhaitables dans le domaine. Nous brosserons alors le tableau de la situation actuelle en outaouais. Une simple énumération des intervenants auprès des entreprises ne suffisant pas, nous tenterons ausi de décrire le champs d'action de chacun d'eux pour mieux discerner leurs forces et faiblesses. Il deviendra alors plus facile, en se rattachant à cette réalité de même qu'aux besoins des petites entreprises, de décrire l'organisme idéal de soutien. 
Au centre de ces réflexions, se retrouve le diagnostic organisationnel de l'entreprise Imagem. L'expérience a débuté en octobre 1987 avec la négociation du mandat, dans lequel les parties s'accordaient sur les objectifs suivants: il s'agissait d'abord pour le chercheur de réaliser un diagnostic organisationnel afin d'éclairer les dirigeants sur la santé globale de l'entreprise. Cette démarche devait ensuite favoriser l'émergence de solutions permettant d'opérer un changement positif en rapport avec cette situation. On allouait deux mois pour l'atteinte de ces objectifs. Dès les premières rencontres, nous pouvions déjà énoncer le contenu problématique de l'entreprise Imagem. Plusieurs faits observés se rattachaient plus particulièrement à la vision de l'avenir de l'entreprise, à l'orientation-production adoptée jusqu'à ce jour, aux ajustements inévitables entre la vente et la production, à l'allocation des ressources humaines et aux orientations futures de l'organisation. Nous posions alors l'hypothèse suivante: les marchés en croissance compliquent la tâche quotidienne des gestionnaires des petites entreprises car ils les obligent à révaluer régulièrement leur stratégie pour tenir compte d'un environnement turbulent et de leurs ressources limitées. Cette hypothèse vaut aussi pour la moyenne et la grande entreprise. Cependant une importante distinction subsiste chez la petite entreprise; ses ressources sont beaucoup plus limitées. Notre hypothèse n'entrait cependant pas en contradiction avec d'autres interrogations: La 
personnalité des dirigeants d'Imagem a-t-elle influencé la santé financière de l'entreprise où est-ce le marché qui en est la source d'influence première? Toute petite entreprise doit fonctionner avec des ressources limitées, mais lesquelles retiennent notre attention chez Imagem? Quelle stratégie l'entreprise a-t-elle adoptée jusqu'à ce jour? cette stratégie tient-elle compte des ressources de l'entreprise? Les dirigeants de l'entreprise connaissent-ils bien leurs forces et leurs faiblesses et en quoi peuvent-ils intervenir de façon éclairée pour améliorer la situation? Tous ces questionnements de notre part trouveront réponse par une méthodologie rigoureuse, que nous reconnaissons dans la méthode des systèmes souples de peter B. Checkland, retenue pour réaliser le présent diagnostic organisationnel. Cette méthode constitue la base du programme de MGPMO de l'UQAT et de plus, elle s'adapte fort bien au type de problèmes que nous rencontrons chez Imagem. C'est une approche systémique qui se définit comme "une approche globale ou macroscopique qui vise à analyser ou concevoir un système dans son ensemble...plutôt que de se buter contre les arbres, ne jamais perdre de vue la forêt"(16). La méthode du professeur checkland ne met pas l'accent sur la réalité externe mais sur les perceptions de la réalité par les intervenants eux-mêmes. Cette méthode facilite la participation active et le consensus des divers intervenants de même qu'elle fait une grande place à la 
personne et à ses valeurs. En outre, cette méthode privilégie "un processus dans lequel les chercheurs et les acteurs, conjointement, investiguent systématiquement un donné et posent des actions, en vue de solutionner un problème immédiat vécu par les acteurs et d'enrichir le savoir cognitif, le savoir-faire et le savoir-être, dans un cadre éthique mutuellement accepté" $(30)$. Ce qui permet à plusieurs tenants de la méthode du professeur Checkland de qualifier celle-ci de recherche-action.

Pour améliorer la compréhension de ce document, nous vous exposons dans les prochains paragraphes les trois grandes divisions et nombreuses subdivisions.

En partie A, nous présentons notre vision de la problématique des petites entreprises en phase de démarrage.

En partie $B$, nous exposons le fruit du diagnostic organisationnel réalisé chez Imagem à l'automne 1987. On peut alors constater que les problèmes auxquels l'entreprise doit s'attaquer, sont surtout reliés à la problématique du démarrage d'entreprise, telle que nous la décrivons dans la partie A. Cette deuxième partie est de loin la plus volumineuse du présent mémoire. 
En partie $C$, nous présentons une dernière série de réflexions issues de notre recherche. Premièrement, nous nous sommes attardés à la méthodologie utilisée. Deuxièmement, nous avons étudié l'impact du diagnostic dans l'entreprise Imagem. puis, nous avons finalement réfléchi sur les moyens que les gouvernements doivent mettre en oeuvre pour soutenir les petites entreprises dans leurs premières années de fonctionnement. 
PARTIE A 
9

I

LA PROBLEMATIQUE DES

PETITES ENTREPRISES EN PHASE DE DEMARRAGE 
La problématique des petites entreprises en phase de démarrage représente un sujet de discussion parfois controversé. Le présent chapitre vise donc à soumettre au lecteur une réflexion d'abord concentrée sur les différents stades de développement de l'entreprise puis sur les caractéristiques de la petite entreprise, sur le profil de son propriétaire et sur les principaux problèmes auxquels elle doit faire face. 
Aux dires du ministre délégué à la PME, Monsieur Vallerand, "les PME dans leur ensemble, affichent de meilleures performances en matière d'emplois que les grandes" (33). Il ajoute d'autre part qu'on ne peut pas oublier la grande instabilité de l'emploi chez elles. Environ cinquante pour cent (50\%) des entreprises ferment leurs portes dans les cinq premières années et "sur vingt PME nouvellement lancées, dix-neuf devront fermer leurs portes en deçà de dix années"(28). Selon les études de Dun \& Bradstreet, quatre-vingt-dix pour cent $(90$ z $)$ des échecs de ces entreprises résultent d'une mauvaise administration. Ces études décrivent comme suit le profil typique d'une entreprise en failite: "elle est dirigée par un seul propriétaire possédant habituellement une formation technique; elle compte moins de trois employés; elle a un actif inférieur à $\$ 100,000$ et un chiffre de vente inférieur à $\$ 200000$. L'entreprise qui Eailit est donc une micro-entreprise"( 10$)$

on peut donc penser que la phase de démarrage d'une entreprise représente une période très critique pour sa survie. Selon Fourcade la phase de démarrage "débute au moment où l'entreprise lance la première sèrie de fabrication commercialisée, ou bien reçoit les premières commandes". (19) pour notre part, nous présumons que cette phase peut s'étendre jusqu'à la deuxième année d'existence, les risques de fermeture demeurent très élevés durant cette période. 
Dans les pages qui suivent, nous nous efforcerons de présenter les éléments majeurs de la problématique des petites entreprises en phase de démarrage, à la lumière de nos expériences auprès de celles-ci. Notre texte sera renforcé à cet égard, par les écrits de cinq auteurs préoccupés par le sujet. Robidoux traite dans son volume Les crises administratives dans les PME en croissance, des sept crises de croissance et leurs conséquences sur l'évolution de l'entreprise. Pour leur part, Perreault et Dell'Aniello exposent leur point de vue des problèmes vécus par les PME et des façons d'y remédier, dans leur volume comment la PME peut survivre aux années 80 . Finalement, dans la revue PMO un article des auteurs Lorrain et Dussault, éclaire notre perception du profil psychologique et des comportements de gestion des entrepreneurs en phase de démarrage.

D'après Lorrain et Dussaut "L'examen de la documentation révèle que les recherches portant sur les spécificités de gestion de la PME ne tiennent pas suffisamment compte des différents stades de développement de l'entreprise, sauf quelques travaux (Fourcade, 1984; Glueck et Vosikis, 1980; Robinson et al., 1984) qui abordent implicitement cette problématique." (19) Ce qui nous amène donc à développer une analogie entre le développement des petites entreprises et celui des êtres humains. Ainsi, les stades de l'enfance, de l'adolescence et de l'âge adulte ne sont pas exclusifs à l'être humain, ils 
sont ausi présents dans le développement de l'entreprise. A titre d'exemple, tous deux vivent le stade de l'enfance où existe le besoin des autres mais où on veut ausi tenter nos propres expériences, développer une certaine indépendance. pour l'entreprise, l'enfance correspond à ce que j'ai qualifié précédemment de démarrage, soit de zéro à deux ans. C'est à ce moment que l'entreprise requiert le concours de nombreuses ressources humaines. Contrairement à l'enfant qui n'a pas à faire de choix parmi celles-ci, l'entreprise doit en faire la sélection sans même être trop capable de juger de leurs compétences et de ses propres besoins. Les besoins financiers se font ausi sentir: beaucoup de choses doivent être achetées dès la première année. C'est ausi l'étape où l'entreprise doit rationnaliser plusieurs de ses décisions, ce qui n'est pas très facile pour un être qui en est encore à l'âge mental de l'enfant.

La petite entreprise et 1 'être humain peuvent aussi vivre leur période d'adolescence s'ils survivent à l'enfance. Ils sont alors de mieux en mieux équipés pour affonter les difficultés. Pour l'entreprise, ce stade de développement s'échelonne sur deux à quatre ans. L'entreprise devient alors consciente de ses forces et faiblesses. Elle prend ainsi des décisions de plus en plus éclairées et son comportement de gestion devient de plus en plus équilibré; les décisions tactiques cédant gradueliement la place aux décisions 
stratégiques. Martinet écrivait à ce sujet: "Les problèmes tactiques appellent très généralement l'attention des décideurs car ils ont des effets directs et perceptibles sur l'entreprise. Il n'en va pas de même pour les décisions stratégiques; il peut s'écouler plusieurs mois, voir plusieurs années avant que les dirigeants ne perçoivent vraiment la nécessité d'une réorientation fondamentale de l'entreprise"(21).

Arrive finalement le stade adulte. I'entreprise, tout comme l'être humain, ressent qu'elle atteint réellement son indépendance. Pour l'entreprise, cette période peut se présenter à partir de sa cinquième année. Le processus décisionnel est maintenant à son meilleur.

Parmi les ouvrages que nous avons consultés, les auteurs Robidoux et wallot ont tous deux émis des réflexions intéressantes sur les stades de développement de l'entreprise. Les exposés de Robidoux sont relativement proches de notre propre vision des choses. Selon lui, les entreprises passent par sept crises de croissance au cours de leur évolution. Ce sont d'abord les phases de lancement, de liquidité et de délégation qui correspondent, à notre avis, au stade de l'enfance tel que décrit plus haut. Viennent ensuite les phases de leadership et de financement, qui se rapprochent 
du stade de l'adolescence. Puis les phases de prospérité et de continuité, qui rappellent le stade adulte.

wallot ajoute une touche fort pertinente à la question, en précisant l'adage qui dit que la petite entreprise est le prolongement de son propriétaire: "Le cycle de vie de l'entreprise est d'abord fonction du cycle de vie du propriétaire. Entre 27 et 34 ans, la plupart des individus mènent trois carrières de front: gestionnaire-père-époux, par ordre décroissant. Entre 35 et 45 ans, on constate un mouvement vers la vie privée pour réparer les frustrations antérieurement créées. A 45 ans et plus, on constate l'intégration de la vie privée et de la vie professionnelle". (34) Dans notre mémoire, nous nous limiterons à la problématique des petites entreprises en phase de démarrage; l'enfance, vécue entre zéro et deux ans.

Selon nous, le plus grand problème auquel doivent faire face les jeunes entreprises, consiste en un manque de ressources humaines de gestion. Souvent fortement centrés sur leurs activités de production, les propriétaires d'entreprise souffrent inévitablement de myopie; ce qui les empêche d'avoir une vision plus systémique de la situation de leur entreprise. Ils sont la plupart du temps inconscients de leurs besoins spécifiques de gestion financière, marketing ou 
autre, et à l'occasion trop sûrs d'eux-mêmes; croyant pouvoir tout organiser seul, sans $1^{\prime}$ aide de personnel compétent. Dans son livre sur les causes des faillites d'entreprise, Parent confirme que "Les causes profondes de l'insolvabilité de certaines entreprises sont d'ordre administratif. Généralement les petites entreprises n'admettent pas leur manque de connaissances et d'expérience en gestion de même que l'utilité et la rentabilité des personnes-ressources extérieures à l'entreprise. Ils refusent toute association avec des investisseurs privés de façon à alléger la structure du capital de l'entreprise". (24) Les conséquences de cette réalité vécue dans les petites entreprises sont nombreuses. Tantôt aux prises avec des problèmes aigus de fonds de roulement, tantôt à court de contrat et tantôt, confrontés à des problèmes de personnel; les propriétaires réagissent tant bien que mal aux difficultés qui les assaillent de toute part. Ces difficultés parfois accentuées par les bouleversements de l'environnement, poussent les propriétaires d'entreprises à prendre des décisions rapides et de nature opérationnelles (ex: embaucher du personnel, négocier un contrat, acheter des équipements, etc). On prend fort peu de décisions stratégiques à ce stade de l'évolution d'une entreprise et malheureusement lorsqu'elles sont prises, on oublie souvent de considérer les ressources financières, 
humaines ou matérielles de l'entreprise à ce moment. On s'engouffre alors dans une crise encore plus aiguë, par un manque flagrant de planification. Dans leur volume sur Ia PME manufacturière, D'Amboise et Gasse écrivaient ceci: "Il existe plusieurs contraintes à la planification dans les petites entreprises. D'abord le fait qu'on a affaire à un one man show qui n'est pas porté à discuter avec d'autres de ses idées et de ses projets. Ensuite la multitude de tâches qu'il doit accomplir le pousse à se consacrer aux domaines qu'il connait le mieux. Finalement, découragé par l'ampleur des recherches à faire dans un processus complet de planification, et pressé par le temps, le dirigeant de petite entreprise a tendance à ne s'occuper que des problèmes immédiats de tous les jours"(9).

Ces considérations rejoignent assez bien l'hypothèse que nous avions émise dès le début de notre recherche, à savoir que les marchés en croissance compliquent la tâche quotidienne des gestionnaires des petites entreprises car ils les obligent à réévaluer régulièrement leur stratégie pour tenir compte d'un environnement turbulent (besoins du marché, situation concurrentielle, technologie, etc) et de leurs ressources limitées. Les dirigeants éprouvent alors des difficultés à évaluer clairement l'importance à accorder aux décisions opérationnelles versus les décisions stratégiques. 
Dès qu'on manque de personnel de gestion compétent, il devient inévitable que les propriétaires de l'entreprise tentent de se découvrir des talents de gestionnaires. Dans les entreprises à plusieurs propriétaires, on choisira ainsi le "moins pire" pour s'occuper des affaires administratives. Celui-ci s'occupera de gestion du personnel, de facturation, d'enregistrement des donnés comptables, de préparation de rapports comptables et il définira des stratégies de vente; tout cela selon ses propres priorités et sa formation. Il deviendra dans certains cas l'homme-orchestre, souvent au détriment de la santé de l'entreprise.

Selon philippe Bernoux "lorsque des entreprises disparaissent en raison des contraintes qui pèsent sur elles, ces échéances mortelles ne sont pas des données incontournables: ce sont les membres de l'entreprise qui n'ont pas su s'organiser pour les contourner. Déposer son bilan en invoquant le marché est une tromperie partielle: les membres de l'entreprise n'ont pas su s'organiser pour survivre. C'est leur organisation qui est coupable, non le marché". (3)

Le second problème que la jeune entreprise rencontre consiste en une mince marge de manoeuvre financière. Elle peut ainsi être consciente de ses besoins en ressources humaines de gestion sans disposer des sommes suffisantes pour redresser la situation. 
De la même façon, avoir besoin d'une campagne de publicité intense sans trouver les fonds pour la financer. on doit faire beaucoup avec peu de fonds. Ce sont les entreprises qui font preuve d'ingéniosité qui réussissent le mieux. Ajoutons aussi que la situation des propriétaires de l'entreprise n'est guère plus stable. ceux-ci ont injecté tout ce qu'ils pouvaient dans le Einancement de départ et leurs maigres salaires des premières années ne leur permettent pas d'améliorer leur bilan personnel afin de soutenir l'entreprise à grands renforts.

Au sujet de la problématique des PME, Robidoux identifie les causes en fonction du stade de croissance des entreprises. Par exemple à la phase du lancement, il entrevoit trois erreurs majeures: le manque d'expérience équilibrée des personnes-clés en gestion (équilibrée faisant référence à un minimum d'expérience dans la vente, la gestion interne, etc, laquelle expérience doit être suffisante pour comprendre certaines interrelations entre les diverses fonctions administratives); un système comptable rudimentaire et une sous-capitalisation au démarrage. Dans la seconde phase soit la crise de 1 iquidité, il identifie un problème d'inexpérience du financement des entreprises dans une période où les stocks et les comptes à recevoir augmentent rapidement. 
Finalement pour la troisième phase soit la crise de délégation, il expose le problème de comportement des gestionnaires qui tendent à vouloir tout contrôler, dans une entreprise qui n'est plus à la mesure d'un seul homme; ou de petitesse de l'entreprise qui ne peut s'entourer d'une équipe de gestionnaires compétents. Selon Robidoux, il ne faut cependant pas perdre de vue que "Dans bien des cas, le problème fondamental n'est pas de nature financière... mais plutôt le résultat de certaines erreurs de gestion qui remontent à plusieurs mois ou à plusieurs années. Le gestionnaire qui a mal utilisé l'argent qu'il avait initialement ne fera guère mieux, règle générale avec tout montant additionnel" (29).

Pour leur part, Perrealt et Dell'Aniello comptent beaucoup sur l'amélioration de la gestion financière de l'entreprise pour contrôler les situations bouleversantes de l'environnement dont il est question dans le présent chapitre. Il faut cependant dire que leur volume a été publié en 1985 et il s'inspire grandement des problèmes vécus par les PME, au débuts des années 1980 . Pour être encore plus précis ajoutons que les deux chapitres consacrés par ces auteurs aux problèmes des PME et aux avenues de solutions, constituent une synthèse des commentaires, réflexions et suggestions émis lors d'un colloque réunissant une dizaine de directeurs de succursales de la Banque fédérale de développement. 
Ils s'entendent pour dire que "la première barrière à franchir est celle de la tendance des entrepreneurs à croire que quiconque est en soi un bon gestionnaire" (10).

Les problèmes de gestion financière se font surtout ressentir par: de mauvaises politiques relatives à toutes les fonctions de l'entreprise, un mauvais rendement de chaque dollar dépensé, un manque de planification financière et de contrôle et une faible productivité.

$\grave{A}$ notre avis, Perreaut et Dell'Aniello soulèvent des éléments très intéressants de la problématique des PME, mais ils accordent un peu trop d'importance à la planification Einancière et à son contrôle et pas assez à la planification et au management stratégique.

\footnotetext{
Ainsi, une entreprise qui préparerait des budgets prévisionnels et des dossiers de financement complets, côtoie inévitablement les risques de fermeture lorsqu'elle néglige les facteurs environnementaux, la concurrence, ses compétences distinctives et les nouvelles technologies de son industrie.
} 
on peut ainsi répondre aux questions relatives aux besoins réels des petites entreprises en phase de démarrage. selon nous, la faiblesse des ressources humaines de gestion représentent le dénominateur commun aux différents points de vue exposés précédemment. Comment une petite entreprise peut-elle supporter les salaires de comptable, spécialiste en marketing, conseiller en personnel, avocat, et autres, sans déstabiliser son fonds de roulement?

Afin d'accroitre ses chances de succès, l'entreprise doit donc prendre un certain recul et solliciter les ressources externes qui peuvent l'aider à traverser les nombreuses phases de croissance de son entreprise. Lesquelles solliciter et pour remplir quel mandat? Voilà d'autres questions auxquelles nous tenterons de répondre dans la partie c du mémoire, après avoir exposé l'exemple concret d'une jeune entreprise qui a vécu des problèmes similaires à ceux que nous avons abordés dans le présent chapitre. 
PARTIE B 
I I

L'HISTORIQUE DE L'ORGANISATION 
Les quelques pages qui suivent, tentent de situer le lecteur sur les événements marquants de la jeune existence de l'entreprise Imagem. D'abord son incorporation, puis son échec avec le projet opton, le redressement du printemps 1987 et Einalement la perte d'une partie de ses équipements lors d'un feu à l'été de la même année. 
L'entreprise Imagem est le prolongement d'une première expérience d'entreprenariat par deux des actuels actionnaires de l'entreprise. En effet, à leurs débuts en 1983, Marc Gendron et Jean-Guy proulx avaient mis sur pied une entreprise de conception et production de documents audiovisuels industriels pour fins de promotion, sensibilisation et formation, sous la dénomination sociale de "Communimage". Bien qu'ils entrevoyaient aussi de développer des projets de conception et de réalisation de qualité "broadcast" c'est-à-dire une qualité qui permet de fournir des produits vidéo aux télédiffuseurs nationaux et locaux, ils ne réalisèrent pas cet objectif et se laissèrent tenter par un autre projet qui semblait tout ausi intéressant dans le but évident de se rendre admissible à la subvention PECEC de l'office de planification et de développement du québec. Ainsi, au cours de la deuxième et dernière année d'opération de Communimage, les deux propriétaires ont èlaboré le système de diffusion "Opton". Celui-ci devait permettre à une organisation de diffuser ses messages publicitaires, promotionnels ou d'information sur des écrans de télévision à circuit fermé et transmettre sa documentation écrite par l'utilisation de présentoirs. Ces écrans et présentoirs pouvaient ausi bien être installés dans des centres commerciaux que dans des salles d'expositions. Les coûts de diffusion par mille consommateurs atteints étaient plutôt élevés, soit deux fois plus que ceux de la télévision et trois fois ceux de la radio. 
Ce projet représentait la pierre angulaire sur laquelle devait s'ériger la nouvelle entreprise Imagem. La diffusion de matériel promotionnel, publicitaire et d'information constituait, selon les développeurs, une source indéniable de contrats de conception et production audiovisuelle. Cela devait amener l'eau au moulin. Les investissements initiaux, nécessaires à la bonne marche de l'entreprise s'élevaient alors à $\$ 159000$ dont $\$ 146500$ en équipements de production, de diffusion et de bureau. Le financement était assuré par une subvention de $\$ 70000$ de l'office de planification et de développement du québec, une dette bancaire de $\$ 69000$ et une mise de fonds des actionnaires de $\$ 20000$. C'est à ce moment, à l'été 1985, que les deux fondateurs de l'entreprise se sont associés un troisième partenaire, pierre st-Denis pour l'incorporation de l'entreprise Imagem. La mise en marché d'opton se concrétise dès l'automne 1985 mais les résultats se font attendre un, deux, six mois, puis c'est la désillusion. on constate que le projet ne fonctionne pas. On se trouve alors devant un dilemme; abandonner opton, vendre les équipements à un coût réduit et réorienter la mission de l'entreprise; ou encore poursuivre les efforts de vente du système opton et développer de nouveaux marchés. On retient cette dernière solution.

Au printemps 1987, un redressement de la situation est 
rendu nécessaire suite aux pressions exercés par les créanciers; banque, fournisseurs, ministères du revenu et autres.

Des ressources externes sont sollicités pour redresser la situation en février 1987. C'est ainsi que des représentants du ministère de la main d'oeuvre et de la sécurité du revenu (MMSR), d'Enjeu-outaouais, du ministère de I'Industrie et du Commerce (MIC) et de l'office de planification et de développement du Québec (OPDQ) interviennent à différents niveaux, conjointement avec les dirigeants d'Imagem pour trouver des solutions applicables. Une somme de $\$ 10000$ est alors injectée par le versement de la dernière tranche de subvention PECEC et la mise de fonds d'un nouvel actionnaire, Daniel Brazeau. Il comble ainsi le départ de pierre st-Denis.

Voici la situation du printemps 1987:

La mission retenue consiste à :

- offrir des services techniques en montage et tournage vi déo;

-louer les équipements audiovisuels en main;

-développer le marché industriel et commercial de la conception et production de vidéos et diaporamas.

Les objectifs se définissent comme suit:

-rentabiliser au maximum l'utilisation des équipements en main; 


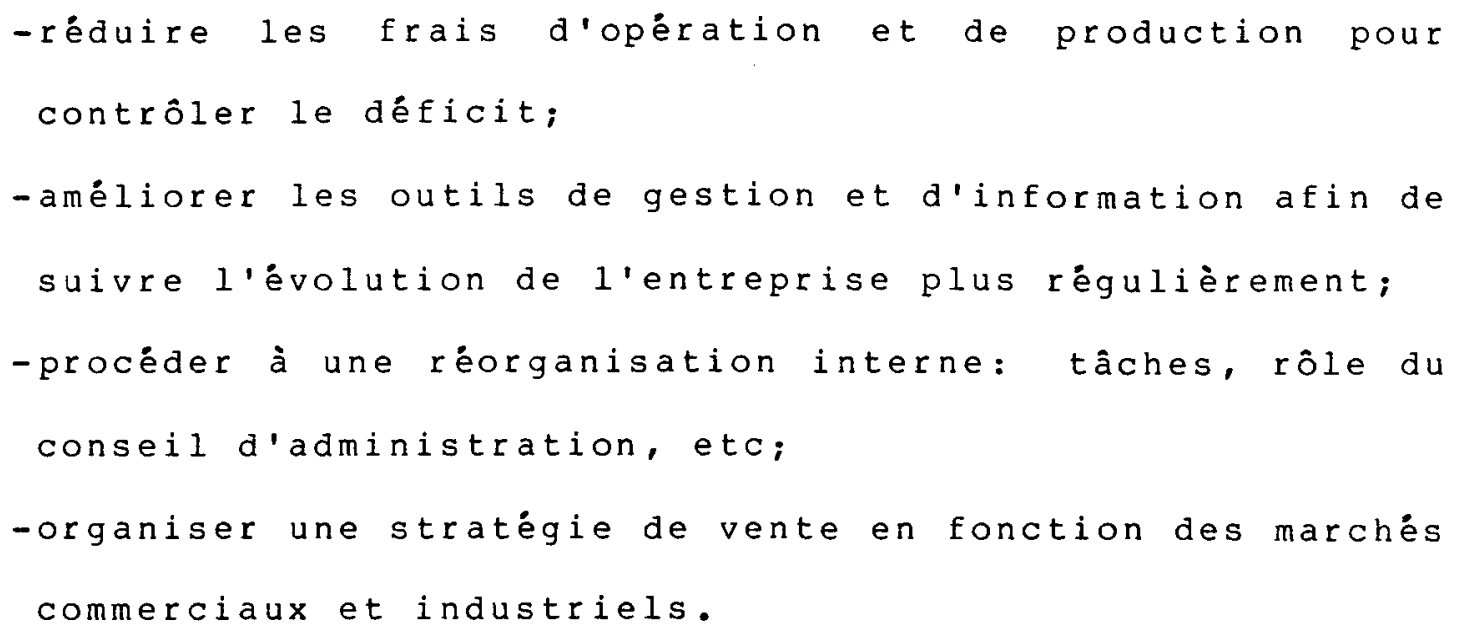

Au début de juillet 1987, Imagem a été victime d'un incendie. Environ $25 \%$ de ses équipements et matériel de bureau sont perdus. En novembre 1987, une somme de $\$ 13909$ a été versée à titre d'indemité par la compagnie d'assurance. Imagem peut aussi compter sur une deuxième tranche de près de $\$ 13000$ dans les prochains mois. Le feu a provoqué l'aménagement précipité dans des locaux qui répondent mal aux besoins de l'entreprise en terme d'espace de travail. On devrait réintégrer les anciens locaux au début de décembre 1987.

Au 31 octobre 1987, Imagem offrait les produits et services suivants à la clientèle: produits vidéo et diaporamas, kiosques, prises de photos, pochette promotionnelle et divers services techniques. Bien que plusieurs productions vidéo se classent dans la catégorie de moyenne gamme, on constate que la majorité des autres produits réalisés se rangent plutôt dans la catégorie bas de gamme. 
L'historique de l'entreprise en dit assez long sur le comportement de gestion adopté jusqu'à ce jour par ses propriétaires. On répond beaucoup plus aux pressions de l'environnement qu'on ne les devance.Ainsi, dans un premier temps, on met sur pied le projet opton afin d'obtenir la subvention du programme PECEC, tout est orienté en fonction de ce projet. Puis au printemps 1987, les pressions exercés par les créanciers obligent l'entreprise à opérer un redressement rapide. Finalement le feu et la situation financière encore instable à l'automne 1987, font croire aux propriétaires de l'entreprise qu'un diagnostic organisationnel pourrait les éclairer. 


\section{I I}

L'ETUDE DE L'ENVIRONNEMENT 
L'étude de l'environnement se révèle la démarche primordiale du diagnostic organisationnel. Le présent chapitre contribue d'abord à faciliter la compréhension par le lecteur des divers facteurs socio-culturels qui influencent le marché de l'audiovisuel, auxquels s'ajoutent les facteurs économiques. Ces derniers seront abordés sous le thème de: structure économique régionale et industrie du film (le marché, la technologie, les ressources humaines, la concurrence, etc). Nous apporterons ensuite quelques précisions sur les environnements politique, légal et physique dont les effets sur l'entreprise ne peuvent être ignorés malgré leur impact moins significatif. Finalement, nous soumettrons au lecteur le résumé des rencontres effectuées auprès de personnes impliquées dans le milieu de la publicité et de l'audiovisuel, afin de connaitre leur perception d'Imagem. 


\section{ENVIRONNEMENT SOCIO-CULTUREL}

Les sociétés nord-américaines ont subi de nombreux bouleversements en ce qui a trait aux moyens de communication. Ainsi les médias électroniques ont substitué une partie du marché de la publicité à la presse écrite. De même, le temps d'exposition du consommateur à la télévision s'est accru considérablement. L'intérêt croissant des québécois pour la vidéo, le diaporama et les autres supports de communication visuels, se réflètent aussi dans la multiplication de journaux et revues spécialisées qui traitent de ces sujets: Télévision, Broadcast, Playback, Qui fait quoi, Plein cadre, etc.... L'image est donc devenue un outil privilégié pour capter l'attention du consommateur ou d'un interlocuteur.

Au Québec comme dans l'ensemble du Canada, on assiste aussi à la prolifération d'organismes sans but lucratif dont le rôle est la sensibilisation et l'information. On les retrouve dans des domaines ausi variés que les regroupements de femmes, les communatés ethniques, les groupes écologiques, etc. Leurs efforts soutenus pour rejoindre le grand public se traduisent souvent par des documents audiovisuels.

Une autre tendance sociale fort prisée depuis quelques années consiste en l'accroissement des ressources humaines, 
matérielles et financières dans des activités de prévention. On n'a qu'à penser à la mission élargie des centres locaux de services communataires, des corps policiers, du ministère de la santé, etc. Ces activités de prévention s'appuient de plus en plus sur des supports audiovisuels.

Ajoutons ausi que le contexte socio-culturel de l'outaouais se différencie considérablement des autres régions du Québec. Ainsi, la cohabitation des populations francophones et anglophones qui le composent de part et d'autres de la frontière Québec-ontario, influence le comportement des consommateurs ausi bien dans le choix de leur station télévisée que dans l'acquisition de biens de consommation. Le fait français n'est cependant pas négligeable.

En outre, n'oublions pas que la Communaté régionale de l'outaouais se développe rapidement. En moyenne depuis 1971 sa population croît une fois et demie à deux fois plus rapidement que l'ensemble du Québec. 


\section{ENVIRONNEMENT ÉCONOMIQUE}

\section{1 structure économique régionale}

Avec une population d'environ 290 000 habitants, l'outaouais québécois connaît une activité économique relativement faible dans son secteur privé mais élevée dans ses secteurs public et para-public. Nous y retrouvons en 1987 , cent cinquante-quatre (154) entreprises oeuvrant dans le secteur primaire; soit deux fois moins qu'en Abitibi ou dans le Bas Saint-Laurent/Gaspésie/Iles-de-la-Madeleine et trois fois moins qu'au Saguenay/Lac-saint-Jean, pour ne considérer que les régions les plus semblables. En ce qui concerne le secteur manufacturier, le nombre est plus encourageant avec neuf cent trente-trois (933) entreprises, soit deux fois plus qu'en Abitibi ou dans le Bas-Saint-Laurent/Gaspésie/Iles-dela-madeleine tout en étant comparable à la région du Saguenay/Lac Saint-Jean. Finalement, pour ce qui est du secteur tertiaire, nous comptons deux mille huit cent dix-huit (2818) entreprises; ce qui est comparable à. l'Abitibi et au Bas Saint-Laurent/Gaspésie/ Iles-de-1a-Madeleine mais près de deux fois moins qu'au Saguenay/ Lac Saint-Jean. (Ces données sont tirées du Rapport du ministre délégué aux PME, 1987.)

Toutes ces statistiques n'empêchent tout de même pas les économistes d'affirmer que le territoire de la communaté 
régionale de loutaouais connat depuis quelques années une recrudescence de l'activité économique privée. Cette effervescence s'est beaucoup fait sentir dans l'industrie de la construction et même durant 1 a récession des années 80 . Par exemple, en 1984, on a mis en chantier six fois plus d'unités de logement qu'en 1980, alors que l'augmentation moyenne québécoise se situe à 40.78 . On peut aussi ajouter que les consommateurs $y$ bénéficient de salaires au-dessus de la moyenne provinciale pour combler leurs besoins et que le taux de chômage est de 5.58 , soit $3.5 \%$ de moins que la moyenne provinciale, selon les données de statistiques canada en février 1988. Les plus gros employeurs se retrouvent aux deux paliers de gouvernement provincial et fédéral.

Ces données à caractère économique, jumelées à une augmentation soutenue de la population, laissent donc croire à un marché en croissance à plusieurs niveaux de l'activité économique sur le territoire de la communaté régionale de 1 'outaouais.

\subsection{Industrie du film}

2.2.1 L'industrie de façon générale

$$
\begin{aligned}
& \text { L'industrie du film se divise en deux secteurs } \\
& \text { d'activités majeurs: l'industrie du cinéma et l'industrie }
\end{aligned}
$$


audiovisuelle; la première se distinguant de la seconde du fait que sa production se destine particulièrement aux salles. L'industrie audiovisuelle, de son côté, regroupe le vaste marché éducatif qui inonde les écoles, le marché de l'information, le marché de la promotion et de la publicité, et celui de la formation.

Jusqu'à ce jour, les deux paliers gouvernementaux par l'entremise de Téléfilm Canada et de la Société générale du cinéma ont surtout concentré leur aide financière dans l'industrie du cinéma. Par exemple, Téléfilm Canada bénéficie d'un budget de près de soixante millions de dollars ( $\$ 60 M)$ pour 1987-1988, dans le cadre de son Fonds de développement pour la production d'émissions canadiennes.

Pour leur part, les télédiffuseurs intensifient leur demande pour des productions audiovisuelles de maisons indépendantes. Selon le président de la maison Production SDA: "l'industrie fílm/vidéo est une industrie de pendule. Au début des années 70, on ferme complètement les vannes de la production TV et puis quelques années plus tard, au début des années 80, on refait marche arrière vers l'industrie privée!" (12)

A titre d'exemple, les réseaux Radio-québec et Quatre saisons démontrent actuellement un intérêt marqué, soit à développer des activités conjointes avec les producteurs 
indépendants, soit à leur donner des productions complètes par appels d'offre. Radio-Canada et Télé Métropole abondent dans le même sens. N'oublions pas que les revenus publicitaires stagnent alors que la fragmentation des auditoires se poursuit entre ces quatre télédiffuseurs. Ceci entraine inévitablement une réorganisation plus ou moins importante chez ceux-ci. Le phénomène du "sponsorship" ou commanditaire constitue un bel exemple du glissement des budgets publicitaires des entreprises vers la promotion. Plusieurs entreprises considèrent maintenant que l'industrie du film peut devenir un bon véhicule promotionnel; citons le mariage réussi de "Lance et compte" et de la compagnie ultramar.

L'industrie de l'audiovisuel peut aussi compter sur les besoins accrus des organismes sans but lucratif, publics et para-publics, au niveau des supports de communication tels que la vidéo et le diaporama, pour rejoindre leur clientèle cible.

Inévitablement, tous ces changements de l'industrie du cinéma et de l'audiovisuel contribuent depuis quelques années à la naissance de nombreuses maisons de production indépendantes. Pour n'en citer que quelques-unes: pixart, Conceptel, Challenge, première, pram, Clip-image, productions 24-30, etc. Derrière certaines d'entre-elles, "se trouvent des gens de télévision d'expérience, des proches collaborateurs des tẻlédiffuseurs ou d'anciens réalisateurs permanents" (11). 
Voici quelques exemples de leur champ d'action: Pixart a développé le concept de l'émission Premières à Quatre saisons; Pram réalise l'émission comptant content au même réseau; et Productions 24-30 co-produit avec des maisons de productions telles qu'Imagem la série Vidéotour à Radio-Québec. La rubrique T.V. Action de la revue Qui fait quoi donne ausi un bon indice de l'effervescence de ce marché: à chaque mois une cinquantaine de productions de maisons indépendantes $y$ sont décrites sommairement. Il ne faut toutefois pas se leurrer: le marché de l'audiovisuel se comporte différemment dans les régions périphériques. D'une part, la structure économique y est beaucoup moins développée et les sièges sociaux d'entreprises d'envergure se font rares. D'autre part, toute l'industrie des communications gravite autour du Montréal métropolitain, provoquant ainsi un effet d'entraînement fort appréciable sur l'industrie audiovisuelle de cette région leffet qui n'est pas toujours évident pour une région comme l'outaouais, par exemple).

2.2.2 Les prospectives du marché de l'audiovisuel

En mai 1987, un groupe de travail a été chargé par le ministère fédéral des communications de faire des recommandations quant aux politiques qui pourraient être mises de l'avant pour appuyer le développement de ce secteur. Selon ce comité: "il est extrêmement difficile d'évaluer la taille 
de ce secteur de l'industrie, l'information statistique est insuffisante, incohérente, incomplète ou tout simplement inexistante" (25).

Le comité s'est toutefois aventuré pour tenter une évaluation intéressante bien que partielle, estimant le marché à un peu plus de quarante millions de dollars (\$40M), pour l'ensemble du Canada.

Des données obtenues d'autres sources, pour l'exercice 1987-1988, nous indiquent par exemple que Radio-Québec consacre 258 de son budget total, soit quatorze millions de dollars (\$14M) à des productions indépendantes. Chez Télé Métropole, on y consacre un peu plus de trois millions de dollars $(\$ 3 M)$.

Que dire du centre du film et de la vidéo, organisme acheteur pour de nombreux ministères fédéraux, qui a pratiquement doublé son budget depuis cinq ans, passant de sept millions de dollars $(\$ 7 M)$ en 1984 à treize millions cinq cent mille dollars $(\$ 13.5 \mathrm{M})$ en 1988. Actuellement, cet organisme consacre $80 \%$ de son budget aux productions vidéo comparativement à 408 vers $1 \mathrm{a}$ fin des années 70 . En 1988 , les trois quarts du budget destiné à la production vidéo sont consacrés à des productions de 10 minutes et le reste à des 
émissions télévisées. Aux dires d'un responsable du centre, un produit vidéo typique se définit comme suit: c'est une production de 10 minutes, dont le budget varie entre $\$ 15000$ et $\$ 20000$, toujours effectuée dans les deux langues officielles canadiennes.

2.2.3 La problématique générale du marché de l'audiovisuel

2.2.3.1 L'éducation de la clientèle

La majorité des intervenants du domaine de l'audiovisuel s'entendent pour dire que le marché est encore en phase de croissance en ce qui a trait aux produits de qualité. Il reste encore beaucoup d'éducation à faire dans le marché institutionnel, industriel et commercial. En effet, bien qu'un nombre grandissant d'organisations aient recours à la vidéo, au diaporama ou à d'autres supports visuels de communication pour leur campagne de publicité, de promotion ou d'information; peu d'entre-elles sont prêtes à payer pour un produit original et de qualité. Par exemple, dans le cas de produits vidéo, elles s'improvisent souvent comme scénaristes pour ensuite demander à des agences de publicité ou maisons de productions audiovisuelles de réaliser le concept, c'est-àdire, faire le tournage, le montage et la post-production. Il en résulte un produit peu professionnel. 
Les entreprises qui acceptent un tel contrat voient alors leur nom associé à des vidéos de faible qualité et risquent de s'écarter des productions de moyenne et haute gamme beaucoup plus exigentes, mais aussi beaucoup plus rentables. En bref, si on part du principe que les productions réalisées constituent l'outil à privilégier par les maisons de productions audiovisuelles et les agences de publicité pour se tailler une place dans le marché, quelques années de productions médiocres pourraient alors suffire à les exclure du marché de moyenne et haute gamme.

Les maisons de productions audiovisuelles doivent donc redoubler d'effort pour convaincre le client potentiel d'investir une somme raisonnable si elles veulent réaliser une production originale et professionnelle. on substitue ainsi, petit à petit, le marché de moyenne et haute gamme, au marché bas de gamme occupé par certains télédiffuseurs locaux et par de nombreux pigistes.

\subsubsection{La technologie}

Les équipements, de même que les méthodes de productions audiovisuelles évoluent constamment et cette réalité se traduit par des normes de qualité de plus en plus sévères pour les productions de moyenne et haute gamme destinèes à la 
télẻvision. On parle de qualité "broadcast". On assiste alors à un élévation des barrières à l'entrée de ce domaine spẻcifique; les coûts de la technologie étant très importants. Certaines maisons de production se tourneront inévitablement vers le marché institutionnel pour rentabiliser leurs équipements et d'autres tenteront de se l'accaparer en misant plutôt sur leurs ressources humaines. Les maisons de production de type artisanal ont peu de chance si elles misent sur leurs équipements, car elles n'ont ni les ressources financières pour renouveler régulièrement les équipements désuets, ni le chiffre d'affaires suffisant pour les rentabiliser en si peu de temps. Un minimum d'équipement de base peut toutefois leur permettre de minimiser les coûts de location à l'extérieur. Dans cet optique, on peut donc supposer que les grandes maisons de production auraient avantage à côtoyer les petites maisons de production et vice versa, les premières tentant de maximiser l'utilisation de leurs équipements et les secondes de minimiser leurs coûts de production.

\section{$2 \cdot 2 \cdot 3 \cdot 3$ Les ressources humaines}

Une bonne partie des ressources humaines des petites maisons de production ne bénéficient pas de permanence; on les embauche à titre de pigistes, pour chaque nouveau contrat négocié. Cette façon de faire comporte un avantage marqué 
puisque ça permet une plus grande flexibilité entre les besoins du moment et les disponibilités financières. Cependant, on ne peut négliger l'effet négatif que cette situation entretient sur le degré d'appartenance du personnel pigiste envers l'entreprise.

2.2.3.4 La segmentation du marché

La segmentation du marché peut se définir en fonction de deux paramètres conjoints; l'utilisation et la qualité du produit.

a) Utilisation du produit

On distingue deux façons d'utiliser le produit:

- Le produit vidéo faisant partie d'une campagne publicitaire complète. Le client ne se contente pas d'un vidéo, il veut un logo, un message original qui s'adapte aussi bien à la presse écrite qu'à la télévision. Le souci de cohérence entre les divers supports de communication est omniprésent. Le client sera alors attiré par les agences de publicité qui sont les plus susceptibles de lui fournir des services complets. 


\begin{abstract}
-Le produit vidéo en tant que message publicitaire et promotionnel distinct.

Le client est plus ou moins soucieux de la cohérence entre ses divers supports de communication. Il aura possiblement négocié des services d'imprimerie pour les dépliants d'information, des services de graphisme pour le logo et tout cela à quelques mois d'intervalle et chez des fournisseurs distincts. Il arrive ausi que le client dispose de sa propre équipe marketing. Il pourra alors clairement définir ses besoins. Ces deux types de client proviennent du secteur public, para-public ou privé. Le client sera attiré ausi bien par les maisons de productions audiovisuelles que par les agences de publicité.
\end{abstract}

b) Qualité du produit

Il existe trois catégories pour la qualité du produit:

-Les produits bas de gamme.

Le client ne veut pas investir des sommes élevés ni pour l'ensemble de la campagne publicitaire ni pour le document audiovisuel.

-Les produits de moyenne gamme.

Le client veut investir une somme raisonnable sans 
exagérer, que ce soit pour une campagne publicitaire complète ou pour un document audiovisuel.

- Les produits haut de gamme.

Le client est emballé par les possibilités d'une campagne publicitaire de prestige et/ou d'un document audiovisuel original et professionnel. Il est prêt à payer un montant substantiel.

Le tableau synoptique suivant illustre les caractéristiques de la segmentation du marché. Certains éléments, tels que le prix de vente des produits, sont spécifiques au marché audiovisuel de l'outaouais. La définition des caractéristiques du marchẻ audiovisuel de l'outaouais repose sur une évaluation arbitraire. 
TABLEAU 3.1 Segmentation du marché

BAS DE GAMME

MOYENNE GAMME

HAUT DE GAMME

\begin{tabular}{|c|c|c|c|}
\hline $\begin{array}{l}\text { CAMPAGNE } \\
\text { PUBLICI- } \\
\text { TAIRE } \\
\text { COMPLÈTE }\end{array}$ & $\begin{array}{r}\text { fournisseurs:-pigistes } \\
\text {.agences de } \\
\text { publicité }\end{array}$ & \begin{tabular}{|l} 
fournisseurs : . agences de \\
publicité
\end{tabular} & $\begin{array}{r}\text { fournisseurs: . agences de } \\
\text { publicité }\end{array}$ \\
\hline $\begin{array}{l}\text { PRODUIT } \\
\text { AUDIO- } \\
\text { VISUEL } \\
\text { DISTINCT }\end{array}$ & $\begin{array}{r}\text { fournisseurs : } \text { pigistes } \\
\text {.stations } \\
\text { de T.V. } \\
\text {.maisons de } \\
\text { production } \\
\text { client: .ent. privées } \\
\text {.org. sans but } \\
\text { lucratif } \\
\text {.institutions } \\
\text { publiques et } \\
\text { para-publiques } \\
\text { demande: élastique } \\
\text { prix de } \\
\text { vente: } 2000 \$ \text { à } 7000 \$\end{array}$ & $\begin{array}{r}\text { fournisseurs: } \begin{array}{r}\text { maisons de } \\
\text { production } \\
\text {.pigistes } \\
\text { réputés }\end{array} \\
\text { client: ent. privées } \\
\text {.org. sans but } \\
\text { lucratif } \\
\text {.institutions } \\
\text { publiques et } \\
\text { para-publiques } \\
\text { demande: } \pm \text { élastique } \\
\text { prix de } \\
\text { vente: } 7000 \$ \text { à } 15000 \$\end{array}$ & $\begin{array}{r}\text { fournisseurs: } \begin{array}{r}\text { maisons } \\
\text { de pro- } \\
\text { duction } \\
\text {-pigistes } \\
\text { réputés }\end{array} \\
\text { client: .ent. privées } \\
\text {.org. sans but } \\
\text { lucratif } \\
\text {.institutions } \\
\text { publiques et } \\
\text { para-publiques } \\
\text { demande: peu élastique } \\
\text { prix de } \\
\text { vente: 15000\$ et plus }\end{array}$ \\
\hline
\end{tabular}


c) Parmi les autres éléments à considérer dans la segmentation du marché, ajoutons le facteur linguistique. Pour la région de la capitale nationale par exemple, le marché se subdivise en un marché francophone, anglophone et bilingue des produits audiovisuels. Les marchés anglophones et bilingues sont amplement desservis par de nombreuses entreprises d'ottawa qui offrent aussi leurs services du côté québécois. Par contre, une seule entreprise québécoise, en l'occurence Imagem, offre des services francophones pour le marché québécois. Ce qui ne l'empêche pas d'offrir aussi des services bilingues.

Finalement, les facteurs clés de succès des nombreux produits offerts par Imagem jusqu'à ce jour, méritent une attention particulière.

Nous les exposons dans le tableau 3.2 suivant: 
TABLEAU 3.2 Facteurs clés de succès par produit

\begin{tabular}{|c|c|}
\hline Produits & Facteurs clés de succès \\
\hline Vidéo industriel & $\begin{array}{l}\text { - originalité } \\
\text { - fierté rattachée au produit } \\
\text {.réponse aux attentes du client }\end{array}$ \\
\hline $\begin{array}{l}\text { Vidéo "broadcast" } \\
\text { (fiction) }\end{array}$ & $\begin{array}{l}\text {-qualité du scénario } \\
\text { - originalité sur le plan visuel } \\
\text {.réalisation professionnelle }\end{array}$ \\
\hline $\begin{array}{l}\text { Vidéo "broadcast" } \\
\text { (publicité) }\end{array}$ & $\begin{array}{l}\text {. crédibilité de la maison de production } \\
\text {-qualité et originalité du produit } \\
\text {-respect des échéanciers } \\
\text { - contacts avec le milieu publicitaire }\end{array}$ \\
\hline Diaporamas & $\begin{array}{l}\text { - prix } \\
\text { - crédibilité de la maison de production } \\
\text { - réponse aux attentes du client }\end{array}$ \\
\hline Kiosques & $\begin{array}{l}\text {. originalité graphique } \\
\text {.transmission de l'information }\end{array}$ \\
\hline Photographies & $\begin{array}{l}\text {-prix } \\
\text {-qualité du produit } \\
\text {-services à la clientèle }\end{array}$ \\
\hline $\begin{array}{l}\text { Services } \\
\text { Techniques }\end{array}$ & $\begin{array}{l}\text {.diversité d'équipements } \\
\text {. services à la clientèle }\end{array}$ \\
\hline $\begin{array}{l}\text { Travaux } \\
\text { d'imprimerie }\end{array}$ & $\begin{array}{l}\text {.originalité du concept graphique } \\
\text {-qualité d'impression } \\
\text {.délais de livraison }\end{array}$ \\
\hline
\end{tabular}


Tous les éléments exposés précédemment doivent permettre aux maisons de production audiovisuelle de fixer des stratégies de vente et de promotion adaptées à la segmentation du marché et aux ressources de leur entreprise.

\section{2 .3 .5 La concurrence}

Dans le secteur de la conception et de la production audiovisuelle, la concurrence peut s'exercer à toutes les étapes de fabrication du produit. Par exemple, dans le cas d'une production vidéo s'insérant dans une campagne publicitaire cohérente, c'est l'agence de publicité qui représente le concurrent le plus sérieux. Il n'est par ailleurs pas impossible de devenir un sous-traitant pour l'agence de publicité. Un autre exemple serait le cas de la scénarisation, pouvant être préparée par un pigiste, qui trouve par la suite les ressources de production audiovisuelle nécessaires.

On peut donc catégoriser la concurrence en fonction de la segmentation du produit telle que décrite au tableau 3.1, en ne retenant que les produits francophones ou bilingues.

Dans le bas de gamme:

- les pigistes et agences de publicité pour les campagnes publicitaires ou promotionnelles complètes; 


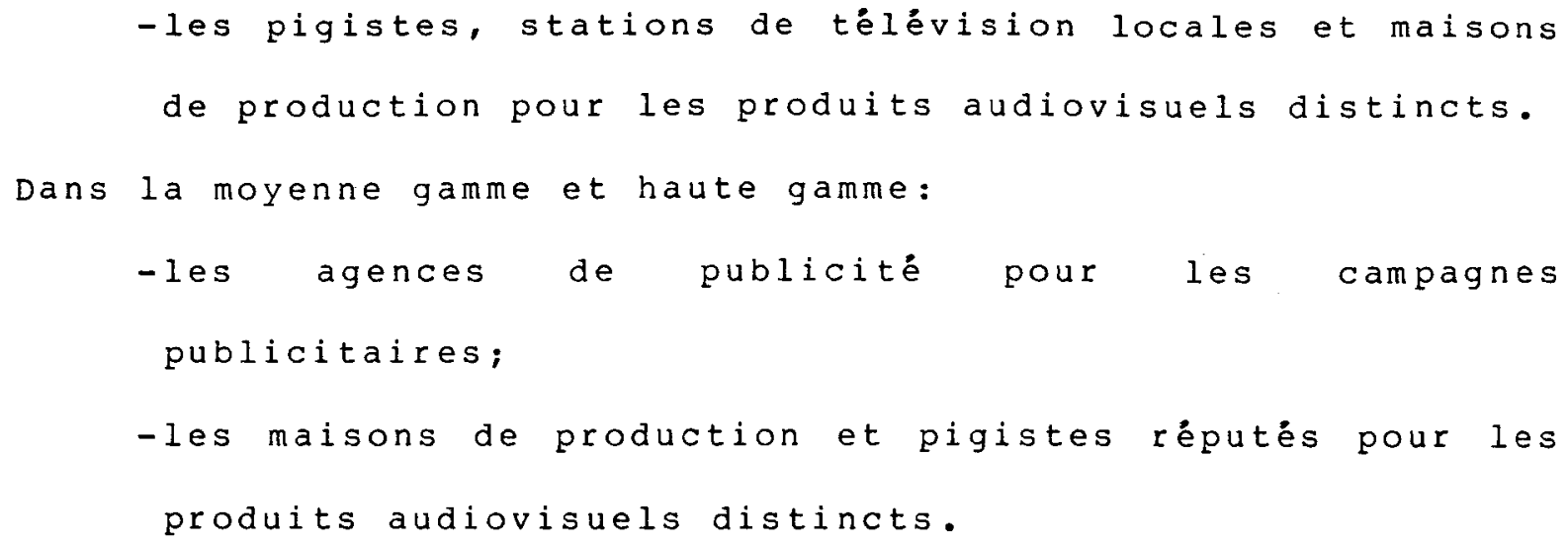

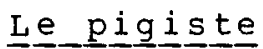

Le pigiste travaille en étroite collaboration avec les agences de publicité ou les maisons de production dans la plupart de ses contrats. Cependant, il comble parfois son calendrier d'activités par des contrats qu'il décroche pour son propre compte.

Le pigiste est normalement peu équipé et il loue les services et équipements de maisons de production locales pour accomplir sa tâche. 
Parmi les pigistes réputés de l'outaouais québécois on connait: François Rouleau, Raymond Charette et Robert Poirier. (voir figure 3.1)

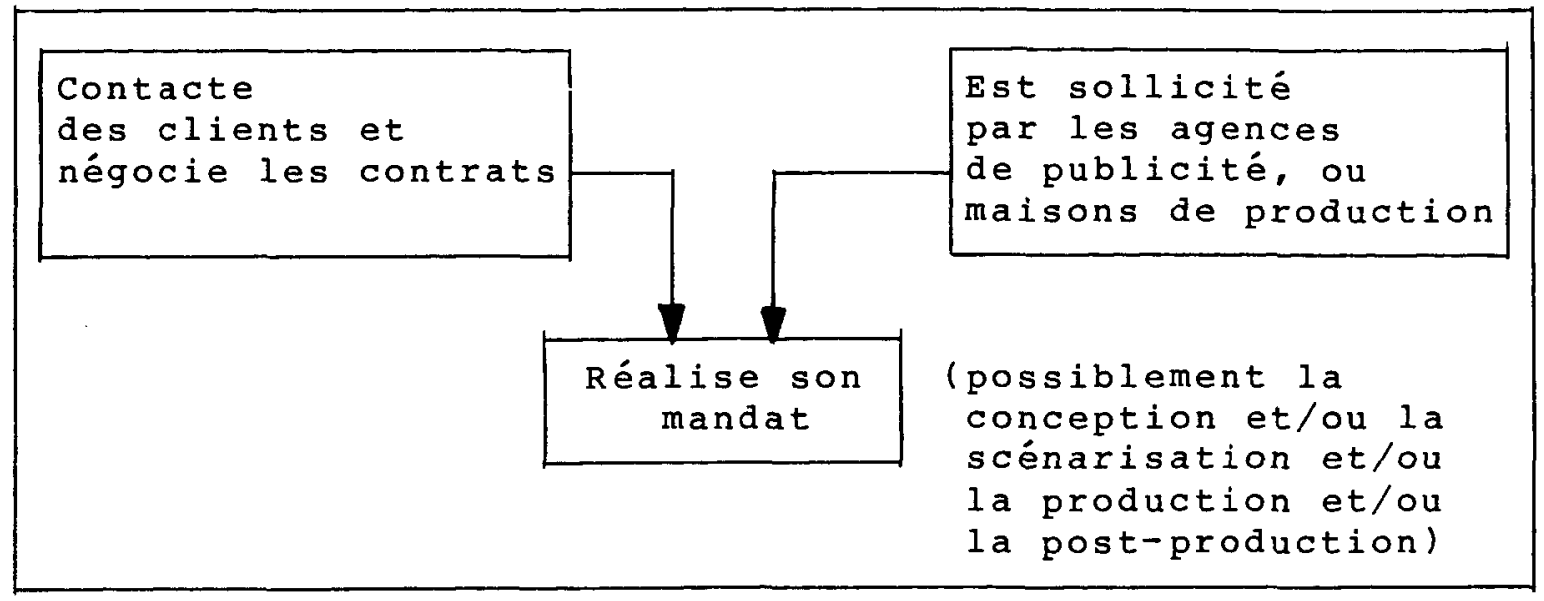

Figure 3.1 Le pigiste 


\section{La station de télévision}

La station de télévision jouit d'un certain avantage sur ses concurrents de l'audiovisuel car elle assure la diffusion des documents. C'est ainsi qu'elle s'accapare une partie du marché de la production.

La station de télévision dispose en effet d'équipements permettant la réalisation de documents audiovisuels. Cependant, son personnel n'a pas toujours la formation pour arriver à un produit professionnel et original.

Dans le milieu, on considère que la station CHOT-TV est active dans ce marché. (voir figure 3.2)

\section{L'agence de publicité}

Les principales activités de l'agence de publicité se situent au niveau de l'évaluation des besoins publicitaires, de la préparation de campagnes publicitaires et du placement média. Toutefois, elle réalise régulièrement une partie non négligeable du document audiovisuel avec son propre personnel, plutôt que de le donner entièrement en sous-contrat.

Les entreprises Impact, Commix, Publicom, Innovacom, Productions Claude Savoie et Les Communicateurs associés 
s'affichent toutes comme agences de publicité de l'outaouais québécois. Certaines opèrent depuis plus de dix ans. (voir figure 3.3 )

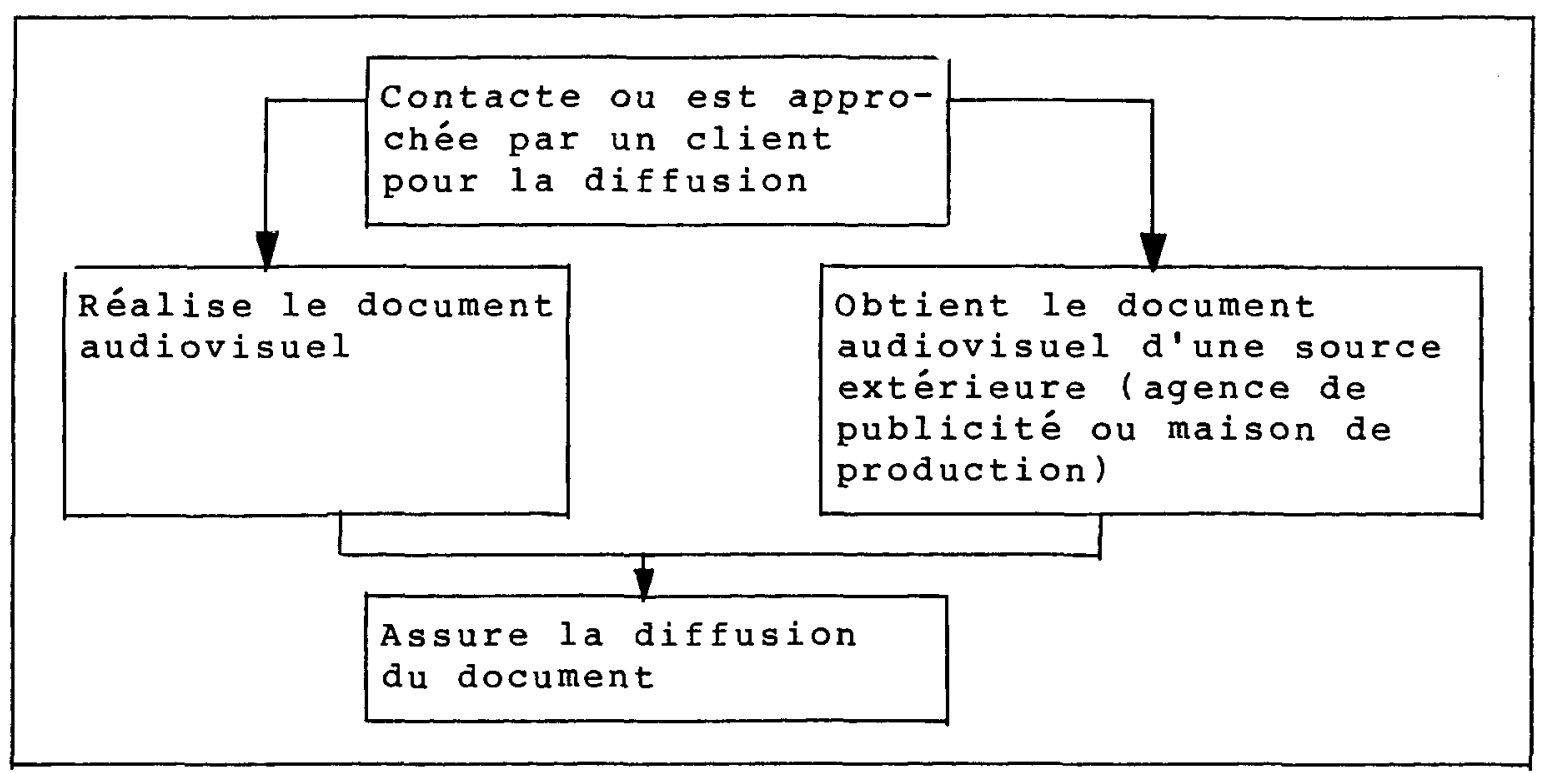

Figure 3.2 La station de télévision 


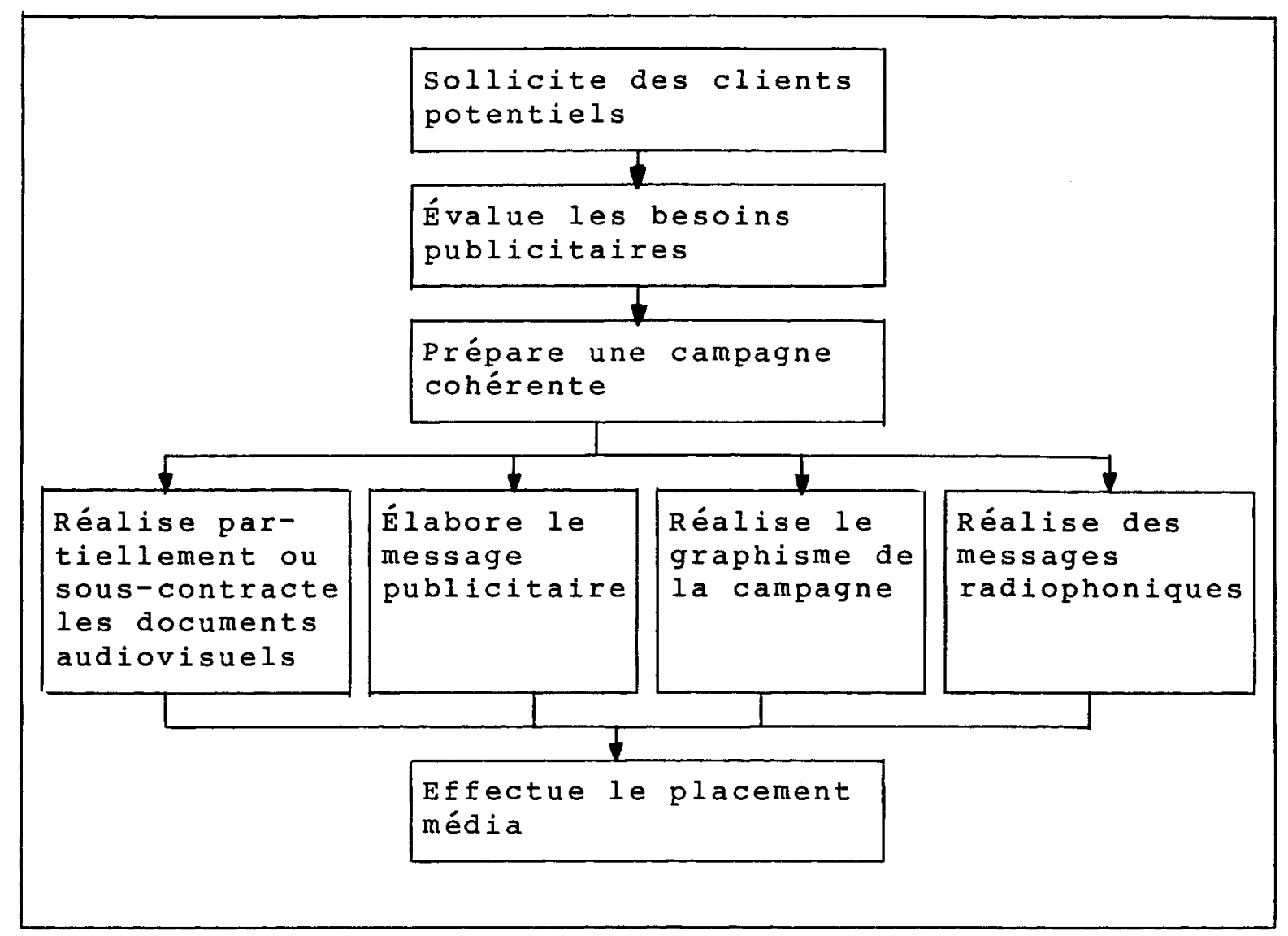

Figure 3.3 L'agence de publicité

\section{La maison de production}

La maison de production obtient ses contrats de deux réseaux: d'abord, par ses propres contacts auprès de la clientèle cible puis, par la sollicitation d'intermédiaires tels que les agences de publicité, les stations de télévision et autres.

Comme il a été expliqué précédemment, Imagem est la seule maison de production dans l'outaouais québécois. Certaines 
maisons de production d'ottawa offrant des services bilingues, doivent cependant être considérées comme des concurrents non négligeables. Les entreprises suivantes Eont partie de ce groupe: Productions Marc, Turnelle production, R. Charbonneau Production et Image Production. (voir figure $3.4)$

Contacte des clients

et négocie les contrats

L



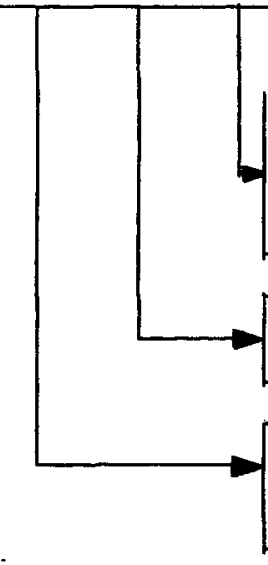

Fait la conception des produits et la scénarisation

Fait le tournage

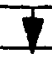

Fait le montage et la post-production
Est sollicitée par les agences de publicité ou stations de télévision provinciales ou pigistes

Figure 3.4 La maison de production 


\section{ENVIRONNEMENT POLITIQUE}

Jusqu'à ce jour, les principales influences de la politique sur le secteur de la conception et production audiovisuelle se concentrent sur les budgets gouvernementaux des divers ministères et autres institutions s'y rattachant. Les politiques gouvernementales semblent toutefois contradictoires. Alors que les budgets de chacun des ministères fédéraux se sont accrus de près de 100 o en cinq ans, (tel que confirmé précédemment par le responsable du centre du film et de la vidéo) ceux des télédiffuseurs nationaux (Radio Canada anglais et français) n'ont pas suivi le même élan.

\section{ENVIRONNEMENT LEGAL}

Les producteurs audiovisuels sont bien sûr soumis aux mêmes normes et réglements que les autres entreprises en ce qui touche l'embauche de personnel (normes de santé et sécurité du travail, retenues à la source, normes du travail, etc) et l'opération d'une entreprise (taxe d'affaires et permis d'exploitation). De plus, ils sont liés à un organisme tel l'Union des artistes du Québec, lequel organisme fixe respectivement les contributions minimales à l'embauche d'un comédien, au fonds de pension des artistes, etc., et les droits à payer sur la musique utilisée dans des productions audiovisuelles. 


\section{ENVIRONNEMENT PHYSIQUE}

La localisation des maisons de conception et production audiovisuelle représente un atout considérable, sans ètre majeur, pour assurer la visibilité de l'entreprise. Il importe de ne pas s'isoler de l'activité économique de la région visée et de bénéficier d'aires de stationnement adéquates. Par exemple, on constate qu'à Hull, les agences de publicité sont installées sur le boulevard st-Joseph ou sur les artères achalandées du vieux Hull. Il en est de même pour la majorité des commerces et restaurants. A Gatineau, les artères Gréber et Maloney regroupent la plus grande partie de l'activité économique. La seule agence de publicité de Gatineau est d'ailleurs installée sur le boulevard Maloney.

Ainsi, lorsqu'on concentre nos activités dans ces secteurs, on s'assure d'ètre à proximité de la clientèle cible.

\section{PERCEPTION D'IMAGEM DANS L'ENVIRONNEMENT}

Une grande majorité des représentants d'agence de publicité, pigistes et autres intervenants (banquier, clients), s'entendent pour dire qu'Imagem est une entreprise jeune qui n'a pas encore fait ses preuves. Les agences de publicité prétendent ainsi ne pas pouvoir donner leur opinion 
sur la qualité des produits offerts, leur originalité et les délais de livraison. Pourtant, ils affirment que ce sont des données nécessaires pour entamer des négociations sérieuses avec Imagem. Certains ont suggéré la préparation d'une production originale et de très bonne qualité, pour prouver ce dont on est capable chez Imagem. Jusqu'à maintenant, Imagem s'est contentée de présenter à ses clients potentiels, un assortiment de ses meilleures productions. On expose donc les réalisations et non les capacités de l'entreprise, en se limitant à la gamme de projets contractés à ce jour.

certains ont ausi été à même de constater qu'Imagem se cherche encore une niche puisqu'elle s'embarque dans des domaines qui ne semblent pas coller avec son image. Par exemple, les kiosques, la photographie et les pochettes promotionnelles. On pense aussi que les équipements d'Imagem sont désuets puisqu'ils ont été achetés selon eux, d'Av plus dont la fermeture serait en partie dûe à la désuétude des équipements. Deux des agences de publicité disent avoir été sollicitées par Imagem mais aucun document concret ne leur a été présenté.

De plus, certains intervenants considèrent qu'une maison de production doit nécessairement développer des projets et tenter de les vendre, car la majorité des clients potentiels 
contactent d'abord les agences de publicité pour l'ensemble des besoins publicitaires, promotionnels et d'information.

Finalement, une majorité d'intervenants rencontrés font confiance au dynamisme et à la capacité de réalisation des dirigeants d'Imagem •

Le développement d'une entreprise comme Imagem est largement conditionné par son environnement socio-culturel et économique. Au niveau socio-culturel, on retiendra que l'image agit maintenant comme un outil privilégié pour capter l'attention des gens. Dans cette optique, on se réjouira de l'impact positif de la prolifération d'organismes sans but lucratif qui adoptent des rôles de sensibilisation, d'information ou de prévention.

Au niveau économique, on constate d'abord que plusieurs marchés de la région de l'outaouais sont susceptibles de subir une forte croissance puisque le développement économique y est soutenu depuis plusieurs années et que sa population croît à un rythme surprenant comparativement à l'ensemble du Québec. Lorsquion concentre notre attention sur le marché de l'audiovisuel, on discerne ausi une forte tendance des télédiffuseurs à faire appel à des ressources extérieures pour réaliser leurs émissions. Ce qui a provoqué ces dernières 
années la naissance de nombreuses maisons de production indépendantes telle qu'Imagem. Cependant, l'effervescence du marché de l'audiovisuel ne semble pas aussi marquée dans la région de l'outaouais qu'à Montréal qui ne peut que bénéficier de la proximité des réseaux importants de télévision et de nombreuses entreprises de toutes catégories et envergures.

Les petites maisons de production font aussi face à deux problèmes majeurs: d'une part l'évolution rapide de la technologie qui contribue à resserrer les normes des produits destinés à la télévision et d'autre part l'élargissement de la notion de concurrence aux stations de télévision locales, aux pigistes indépendants et bien sûr aux agences de publicité.

Finalement, sur la perception que partagent les nombreux intervenants régionaux en audiovisuel en regard de l'entreprise Imagem nous retenons que l'entreprise n'a pas encore fait ses preuves et que l'on connait mal la qualité des produits offerts, leur originalité et les délais de livraison. La majorité des personnes interrogées croient cependant au dynamisme et à la capacité de réalisation des dirigeants d'Imagem. 
LES STRUCTURES 


\begin{abstract}
L'objectif du présent chapitre se présente comme suit: décrire avec justesse les éléments formels qui constituent l'organisation. On $y$ retrouve ainsi des informations sur l'organigramme d'Imagem, les responsabilités et la description des tâches, la compétence des ressources humaines, les politiques de prix et de salaires, et l'aménagement des I i eux.
\end{abstract}




\section{ORGANIGRAMME}

A l'instar de la majorité des jeunes et petites entreprises, il n'existe pas chez Imagem d'organigramme formel. A cet effet, une étude sur les caractéristiques psychologiques et les comportements de gestion des entreprises manufacturières en phase de démarrage indique que "seulement $10 \%$ ont un organigramme formel écrit et $64.3 \%$ affirment avoir un schéma mémorisé de l'entreprise". (19)

Le conseil d'administration, composé des trois actionnaires majoritaires et des deux actionnaires minoritaires, joue un rôle plutôt passif dans la gestion de l'entreprise. Le dernier conseil d'administration a été convoqué lors du redressement du printemps 1987. "Le conseil en tant que façade, existe là où un individu ou un groupe, que ce soit l'équipe dirigeante ou le propriétaire d'une entreprise commerciale, contrôle entièrement l'organisation et choisit de n'utiliser le conseil, ni comme outil, ni comme système de contrôle". (22)

De fait, chez Imagem, la gestion de l'entreprise repose plutôt sur le comité de direction formé par les trois actionnaires majoritaires. Bien qu'on y aborde des sujets de nature tactique ou stratégique, la majorité des discussions 
concernent les activités quotidiennes de l'entreprise. Ce comité se réunit une fois par semaine.

Les pigistes relèvent de Daniel Brazeau et Jean-Guy proulx alors que la comptable relève de Marc Gendron.

Le contrôle de l'entreprise appartient à part égale aux trois actionnaires majoritaires: Marc Gendron, Jean-Guy proulx et Daniel Brazeau. Cinq autres actionnaires minoritaires se partagent 228 des actions.

Le tableau 4.1 fournit la répartition exacte du capitalactions.

Tableau 4.1 Répartition du capital-actions

\begin{tabular}{|lrr|}
\hline & Actions & ordinaires \\
& Nb & 8 \\
Proulx, Jean-Guy & 2682 & 26 \\
Gendron, Marc & 2682 & 26 \\
Brazeau, Daniel & 2682 & 26 \\
Ouimet, Louise & 244 & 2 \\
Houle, Robert & 855 & 9 \\
Morin, Charles & 100 & 1 \\
Bourque, Claude-Julie & 100 & 1 \\
St-Denis, Pierre & T은 $\frac{8}{2} \frac{5}{0}$ & $\overline{9}$ \\
\hline
\end{tabular}

Le registre des procès-verbaux de l'entreprise ne renferme pas les données récentes relatives à la composition du conseil d'administration, la répartition du capital-actions, la nomination du vérificateur et les procès-verbaux. 
2. RESPONSABILITES, DESCRIPTION DES TACHES ET HORAIRE DE

TRAVAIL

\section{1 Responsabilités et description de tâches}

Marc Gendron a comme tâche de coordonner les projets, de faire la représentation auprès des clients et d'assumer les différentes responsabilités de gestion de l'entreprise.

Jean-Guy Proulx assume des tâches qui se rattachent surtout aux activités de production et de marketing de l'entreprise.

Daniel Brazeau assume les tâches de coordination et contrôle de la production, de même qu'il entretient des relations avec les fournisseurs et le personnel de production.

Les pigistes assument les tâches de photographie, de montage, de "post-production" ou autres qui leur sont confiées en rapport avec chacun des mandats négociés. Ces tâches sont normalement reliées à leur formation et leur expérience.

La comptable procède à l'enregistrement des revenus et des dépenses dans le système comptable informatisé. 
Dans les faits, les tâches effectuées par les 3 gestionnaires respectent plus ou moins les cadres élaborés précédemment, à la lecture de documents internes. Ainsi ...

- Marc Gendron ne coordonne pas tous les projets. Il travaille à la production de certains produits tels kiosques, diaporamas, photographies et à l'accomplissement de tâches quotidiennes et routinières (commissions et autres)

- Jean-Guy proulx fait aussi des représentations chez certains clients et il accomplit certaines tâches quotidiennes et routinières.

- Daniel Brazeau ne coordonne et ne contrôle pas toutes les productions. Il accomplit aussi certaines tâches quotidiennes et routinières. $\grave{A}$ loccasion, il sollicite quelques clients.

\subsection{Horaire de travail}

Les trois gestionnaires ont le contrôle quasi-absolu de leur propre horaire de travail. Chacun juge de sa propre contribution à l'organisation et il n'est pas habituel d'en discuter, de comparer et finalement de se remettre en question individuellement. Aucun controle n'est exercé à ce niveau. 
Chaque gestionnaire évalue à près de soixante heures une semaine normale de travail, dans les conditions actuelles.

Les pigistes n'ont pas d'horaire de travail précis. Ils se présentent seulement lorsqu'ils doivent réaliser un contrat. Leur horaire dépend par contre, de la disponibilité des équipements.

La comptable effectue son travail le jeudi de chaque semaine, entre 8 hres 30 et 17 hres.

3. COMPETENCES DES RESSOURCES HUMAINES

\section{1 Gestionnaires}

Jean-Guy Proulx: Il a complété un baccalauréat en communication à l'Université d'ottawa. Il cumule des expériences à titre d'assistant-caméraman, de réalisateur, de professeur en communication et d'assistant de recherche. Il démontre des capacités intéressantes à développer de nouveaux produits et concepts et à réaliser des productions audiovisuelles.

Marc Gendron: Il a complété un baccalauréat en communication à l'Université d'ottawa de même qu'un certificat en administration à l'Université du Québec à Hull. Il cumule des expériences de réalisateur, directeur de production sonore, 
assistant-caméraman, rédacteur et assistant de recherche. Il démontre un intérêt certain pour la gestion de l'entreprise et les relations publiques.

Daniel Brazeau: Il a obtenu un baccalauréat en communication à l'université d'ottawa. Ses expériences de travail se concentrent à trois niveaux: la production d'émissions destinées à la télévision communataire, la conception et production de vidéos et diaporamas, et la vente dans un commerce de détail en électronique. Il démontre des capacités intéressantes à négocier avec les fournisseurs et à réaliser des productions audiovisuelles.

\subsection{Autres ressources humaines}

Monique Lemieux (employée): Elle a obtenu un diplôme d'études collégiales en comptabilité et complète actuellement un baccalauréat en psychologie.

Charles Morin (pigiste): Il a obtenu un certificat d'étude du Collège Algonquin en technique de production et de communication. Il cumule des expériences dans le domaine de la consultation, l'enseignement et la production audiovisuelle.

Claude-Julie Bourque (pigiste): Elle est diplomée en
musique et en techniques d'animation et de réalisation du 
Collège Algonquin. Elle a acquis de l'expérience à titre de coordinateur de projets de communication, de producteur de diaporamas et d'agent de relations publiques.

Chantal Poitras (pigiste): Elle a obtenu un baccalauréat en graphisme. Elle possède peu d'expérience en graphisme cependant elle cumule des expériences dans des secteurs connexes tels que le lettrage et la rédaction de texte.

Autres

pigistes: Nous ne possédons pas de données suffisantes pour décrire la formation et l'expérience de chacun d'entre-eux. Nous exposons simplement leur champ d'intervention.

Constance Alain: rédaction et scénarisation Lucie vigneault: rédaction et scénarisation Benoît Thériault: recherche historique Marc-André Gagnon: tournage vidéo Re né Binet: photographie

4. POLITIQUES OPERATIONNELLES ET REGLEMENTS

\section{1 Salaires}

Depuis le redressement du printemps 1987, les salaires hebdomadaires des dirigeants de l'entreprise sont passés de 
$250 \$$ à l'automne 1986 à $200 \$$ au printemps 1987 puis à $265 \$$ à 1'automne 1987. Ce salaire semble insuffisant pour leur assurer une qualité de vie convenable.

Pour leur part, les cachets des pigistes sont négociés à Ia pièce. Aucun barème n'a été établi à cet effet sauf ce qui concerne les cachets des comédiens, qui sont soumis aux politiques de l'Union des artistes.

\section{2 Politique de prix}

Une Iiste de produits offerts par Imagem, accompagnée de taux horaires, permet aux clients d'évaluer les dépenses qu'ils comptent engager et de comparer les prix avec les principaux concurrents.

Cette 1 iste est insérée dans la pochette d'information de l'entreprise.

\section{3 Heures d'ouverture}

Bien qu'elles correspondent grosso modo aux heures d'ouverture des professionnels faisant affaire avec les institutions et entreprises privées, soit entre 9 et 17 heures, rien n'est clairement décrit à ce sujet. Il arrive assez fréquemment qu'un client ne puisse obtenir de réponse à ses interro- 
gations car les trois gestionnaires sont absents et les individus sur les lieux ne sont pas informés des affaires de 1 'entreprise.

\section{4 Autres éléments de la structure}

Mis à part les signatures de chèques qui sont autorisées par Marc Gendron et Jean-Guy Proulx, les différents contróles financiers et de production ne sont pas clairement définis et connus de tous.

Aucune politique formelle de paiement des fournisseurs n'existe, de même qu'aucune politique formelle de perception des comptes à recevoir.

L'exercice financier de l'entreprise se termine le 30 juin de chaque année. Le bureau comptable chargé de la production des états financiers au 30 juin 1986 et 30 juin 1987 est "Laflèche et Laflèche". Ce bureau entend compléter le tout dans les mois à venir.

\section{AMENAGEMENT DES LIEUX}

Dans ses anciens locaux, Imagem dégageait une image de prestige. Les choses ont changé depuis que le feu a entrainé le déménagement précipité de l'entreprise. Bien que les 
activités de production soient séparées des activités de gestion, un fouilis indescriptible y règne. Ce n'est toutefois que temporaire, car l'entreprise compte réintégrer ses anciens locaux dès décembre 1987 et mettre de l'ordre dans tout cela. Le bail signé avec le propriétaire de ces locaux est d'ailleurs encore effectif jusqu'en mars 1992. La superficie totale représente environ $1400 \mathrm{pi}^{2}$ à un coût mensuel de $\$ 900$. Des espaces de près de 300 pi 2 pourraient devenir disponibles pour sous-location; soit un revenu mensuel d'environ $\$ 350$. Aucune entente écrite n'est cependant disponible à ce sujet. 


\begin{abstract}
on ne doit pas s'étonner qu'lmagem soit si peu structurée; c'est plutôt fréquent dans la petite entreprise. Cependant, si l'absence d'organigramme et de conseil d'administration formel entraîne peu de conséquences négatives sur l'entreprise, il n'en va pas de même pour les politiques salariales, les politiques de comptes payables et recevables qui minent considérablement les chances de survie de l'entreprise.
\end{abstract}

Finalement, retenons que les ressources humaines de l'entreprise Imagem démontrent une plus grande capacité à produire des documents audiovisuels qu'à assurer une saine gestion de l'entreprise. 
LES PROCESSUS 
Dans le présent chapitre, nous tenterons de décrire graphiquement, avec textes à l'appui, les nombreux processus d'activités humaines chez Imagem. On y retrouvera aussi bien des activités de gestion que de transformation; le tout interrelié par des flux d'information, de matière, de personnel ou d'argent. Nous parlerons de deux grands systèmes: le super-système, qui fait surtout référence aux relations entre l'entreprise et l'environnement, puis le système global, constitué des sous-systèmes de planification, de développement et d'opérationnalisation des activités régulières. La lecture de ce chapitre fournira donc au lecteur de nombreux détails sur la façon de faire les choses chez Imagem. 


\section{ENVIRONNEMENT ET SUPER-SYSTEMME}

Les environnements économique, culturel, légal, politique et physique ont été décrits de façon détaillée dans le chapitre II du présent document. La figure 5.1 en rappelle les grandes lignes.

\section{SYSTÈME GLOBAL}

Les principaux processus d'activités qui existent chez Imagem peuvent être regroupés en trois catégories distinctes: les processus de planification stratégique, les processus de développement de nouveaux produits et les processus "d'opérationnalisation" des activités régulières.

La dernière réunion formelle de planification stratégique remonte au printemps 1987. De plus, jusqu'à ce jour, la planification a rarement été confrontée aux résultats obtenus de même qu'aux ressources disponibles dans l'entreprise et aux réalités du marché de l'audiovisuel.

Le développement de nouveaux produits a nécessité de nombreux efforts de conceptualisation et de mise en marché, sans trop porter fruits. Le projet opton en est un exemple. Ce qui est remarquable à ce niveau, c'est que l'entreprise n'a pas utilisé d'appuis extérieurs concrets sur l'ensemble de la 


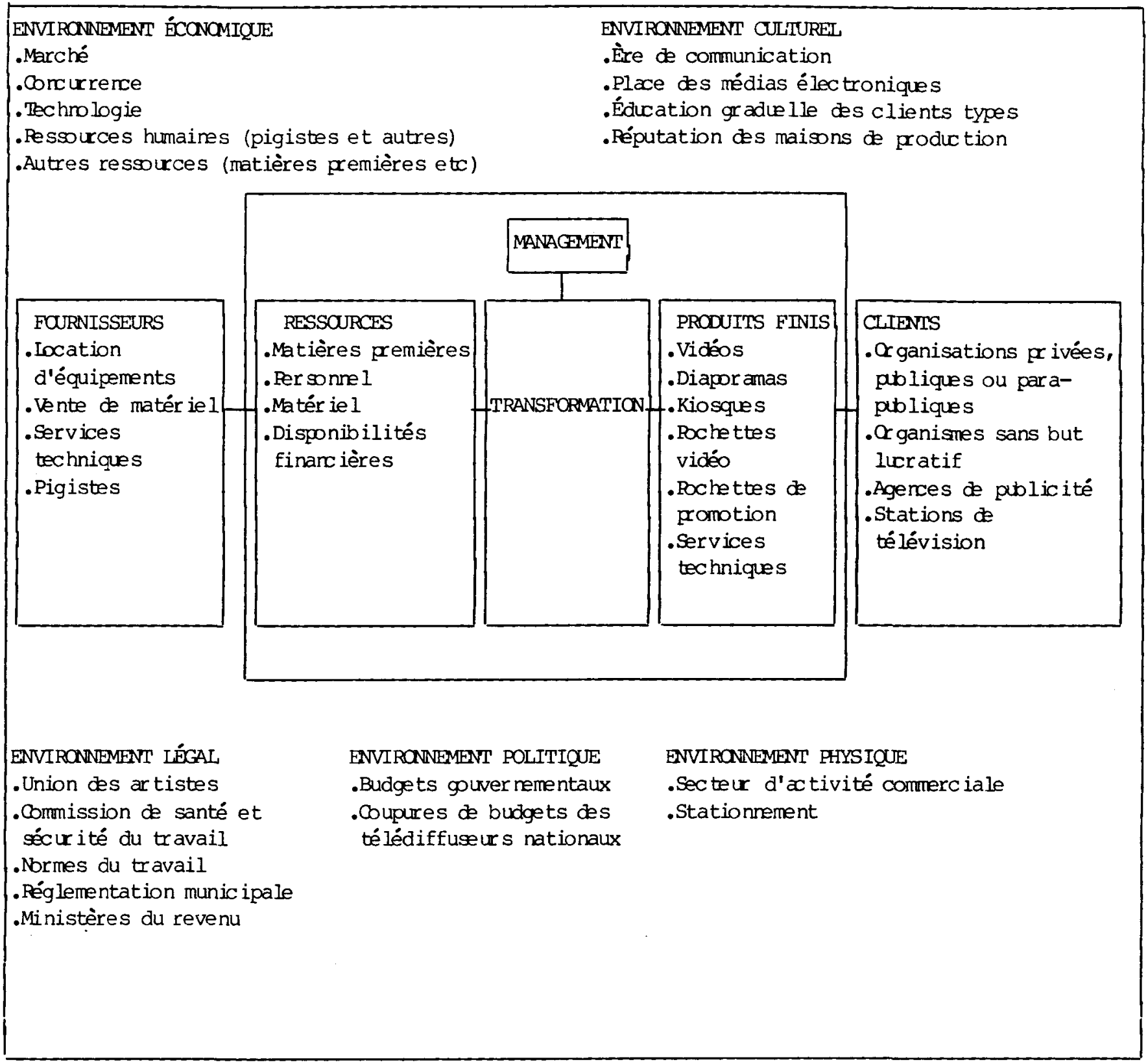

Figure 5.1 Environnement et super-système 
démarche. Pourtant il demeure évident que la mise en marché du produit opton aurait largement bénéficié de l'expérience de firmes spécialisées dans le domaine, que ce soit au niveau d'une étude de faisabilité complète, de la fixation de la date de mise en marché ou des disponibilités supplémentaires nécessaires en terme de ressources humaines et financières.

L'opérationnalisation des activités régulières occupe tout de même la plus grande partie du temps des gestionnaires d'Imagem. En bref, on tente d'une part d'amener l'eau au moulin et d'autre part, de répondre à la demande de la clientèle. L'entreprise éprouve tout de même des difficultés multiples à ce niveau. L'allocation des ressources laisserait place à des améliorations; la qualité des produits n'est pas toujours acceptable; les délais de livraison sont parfois renégociés et le contrôle des opérations présente des déficiences marquées.

Les figures 5.2 à 5.10 traduisent graphiquement le système global de même que les sous-systèmes d'activités de l'entreprise.

\subsection{Sous-système planification stratégique}

Chez Imagem, la planification stratégique est surtout Eaite à court terme, sur 3 à 6 mois. Les deux principales particularités de leur sous-système de planification stratégique sont les suivantes: 
Il arrive une à deux fois par année qu'on réunisse les membres du comité de direction ou du conseil d'administration afin de discuter planification stratégique, mais les propositions qui en découlent n'ont pas vraiment été respectées jusqu'à aujourd'hui.

Les réunions hebdomadaires du comité de direction, normalement prévues pour l'opérationnalisation des activités régulières, donnent fréquemment lieu à de grandes discussions de fond touchant la planification stratégique. Ces discussions amènent parfois des décisions qui seront réalisées concrètement, mais il est fréquent de voir ces discussions s'éteindre à la suite de la simple évaluation des implications, ou encore, à la suite de recommandations d'un gestionnaire. on passe alors à un autre point de l'ordre du jour sans proposer de plan d'action concret et sans nommer de responsable d'un tel plan. on remet inévitablement les choses à plus tard.

\section{2 . 2 Sous-système développement}

Ce sous-système reflétait la position de tous les gestionnaires jusqu'à tout récemment, à savoir qu'il est pertinent pour une maison de production audiovisuelle de développer de nouveaux produits pour assurer sa pérennité. 
Ainsi, deux séries d'activités distinctes se sont développées avec le temps. Il y a bien sûr l'élaboration de nouveaux produits au coeur même de l'entreprise. On entame alors le processus par une réflexion sur les besoins de la clientèle, puis on élabore le concept préliminaire, on en discute avec l'entourage et on procède ensuite à son élaboration complète et finale.

Une autre façon de s'impliquer dans le développement de nouveaux produits, consiste à demeurer ouvert aux propositions d'entreprises compétentes du domaine des communications. Dans ce cas, Imagem ne dirige pas le projet. L'entente avec le groupe MOSAIQUE-GL de Montréal, sur l'élaboration d'un projet de vidéo-disque en est un exemple concret.

Cependant, étant donné l'état actuel des choses, M. Proulx et M. Brazeau semblent vouloir mettre un frein à ce genre d'activités, prétextant d'une part, que cela draine beaucoup d'énergies (et parfois dans des domaines hors du champ de compétence de l'entreprise), et d'autre part, que beacoup reste encore à faire en ce qui concerne "l'opérationnalisation" des activités régulières d'Imagem. 


\subsection{Sous-système promotion et vente}

Pour vendre ses produits, Imagem emprunte deux avenues. D'une part, elle sollicite les clients potentiels par des visites au cours desquelles elle présente ses services et discute des besoins du client. On remet à cet effet une pochette d'information sur Imagem, à tous les nouveaux clients. ذ̀ l'occasion, on leur présente aussi le vidéo promotionnel de l'entreprise. De plus, on répond aux appels d'offre sur les projets intéressants. Finalement, aucune stratégie particulière ne permet de rejoindre certains clients plutôt que d'autres, et les efforts de vente ne sont pas nécessairement orientés vers la mission et les objectifs de l'organisation. D'autre part, l'entreprise entretient des relations publiques en assistant à divers colloques reliés au domaine de la communication, en participant à des concours, etc.

L'ensemble de ces démarches mène inévitablement à la préparation de soumissions par le vendeur. Celles-ci sont préparées à partir de l'expérience du vendeur sans consulter systématiquement l'équipe de production. Elles présentent régulièrement des écarts avec les coûts qui sont réellement encourus, surtout pour les produits de bas de gamme. 


\subsection{Sous-système ressources}

La principale caractéristique de ce sous-sytème réside dans le fait que la majorité des activités qui s'y rattachent, sont effectuées à la dernière minute et à la hâte. Cette situation provoque un état où les ressources humaines régulières, en l'occurence les gestionnaires, ont toujours l'impression d'éteindre des feux. Les trois gestionnaires, à tour de rôle, perdent un temps considérable aux activités de dernière minute.

L'allocation des diverses ressources est d'autant plus difficile depuis qu'Imagem ne paie pas régulièrement ses fournisseurs. Ainsi, la liste des fournisseurs de matériel et équipements se rétrécit peu à peu.

En ce qui concerne l'embauche des ressources humaines, elle est souvent faite de façon unilatérale par l'un ou l'autre des gestionnaires sans tenir compte de l'ensemble des besoins de l'entreprise à ce niveau. Il n'est pas rare de se retrouver avec du personnel ou des pigistes peu expérimentés et peu autonomes qu'on doit former ou suivre de près. on peut alors se demander s'il existe un avantage à opérer à partir de pigistes ou avec du personnel temporaire. Les ressources humaines les plus convoitées par l'entreprise au cours de la 
dernière année appartiennent au métier de: caméraman, photographe, comédien, scénariste, technicien audiovisuel, comptable et graphiste.

En ce qui concerne l'allocation des équipements, plusieurs faits retiennent notre attention:

- pratiquement toutes les productions vidéo de moyenne et haute gamme nécessitent la location d'équipements à l'extérieur de l'entreprise pour le tournage et la post-production;

- pour leur part, les productions vidéo de bas de gamme et les services techniques nécessitent très peu de location d'équipements à l'extérieur de l'entreprise;

- finalement les productions de diaporamas nécessitent peu de location d'équipements à l'extérieur de l'entreprise bien qu'elles soient réalisées avec des moyens de fortune qui entraînent de nombreux frais de main-d'oeuvre.

\subsection{Sous-système production}

Le manque de consultation entre collègues et pigistes, à plusieurs étapes de la production, constitue le dénominateur commun de tous les types de production. 
La communication pure et simple de l'état de la situation des projets, n'est pas plus fréquente. Finalement, peu de coordination est faite entre les différents projets, leurs besoins en ressources et leur calendrier de production. On opère souvent à la dernière minute; 1 à aussi on éteint des feux.

Au niveau de la production, on peut affirmer que la capacité maximale de production correspond à un chiffre d'affaires annuel de près de $\$ 250000$, sur la base des ressources humaines régulières et actuelles de l'entreprise (i.e. les gestionnaires et la comptable).

\section{6 Sous-système mise en marché}

La majorité des activités d'élaboration de stratégies de vente, de cencontre de clients potentiels, de présentation de concepts et de négociation de contrats sont réalisées après l'étape de production du nouveau produit. on engage ainsi des sommes élevées sur un produit que l'on n'est pas vraiment assuré de pouvoir vendre. 


\section{7 sous-système contrôle}

Au niveau du contrôle des opérations, nous constatons que la facturation des gros projets se fait plusieurs semaines après la fin de la production des documents audiovisuels. De même, la perception des comptes à recevoir ne se fait pas de façon systématique. Cette gestion peu rigoureuse des comptes à recevoir occasionne des problèmes aigus de liquidités.

L'enregistrement des données comptables telles qu'achats, paiements, ventes et perception de comptes à recevoir, s'effectue plus régulièrement depuis quelques semaines. Cependant, les derniers rapports d'opération, qui devraient normalement être la suite logique de l'entrée des données comptables, datent de février 1987. Aucun autre rapport complet relatif aux états financiers n'a été transmis par le responsable de I'administration aux deux autres gestionnaires. La comptabilité de l'entreprise est assistée d'un ordinateur à disque dur, compatible avec IBM. Le logiciel utilisé se qualifie, selon le vérificateur actuel de l'entreprise, comme le plus performant qui existe sur le marché.

Cependant, si on dispose depuis un certain temps du logiciel de comptabilité du grand-livre et du journal auxiliaire des comptes à payer, on n'y a pas encore intégré le 
journal auxiliaire des comptes à recevoir. En ce qui concerne le journal auxiliaire des comptes à payer, il demeure incomplet ne tenant pas compte des anciens comptes à payer, remontant au-delà de juin 1987 . 
L'examen des processus d'activités de l'entreprise Imagem laisse donc entrevoir cinq types d'activités qui peuvent nécessiter des améliorations.

D'abord, la planification stratégique s'effectue sans tenir compte des forces et des faiblesses de l'entreprise et elle se rattache très peu à l'opérationnalisation des activités régulières. Pour sa part, le développement de nouveaux produits ne s'appuie pas suffisament sur des études sérieuses; on consulte peu à ce niveau. Le même genre de problème se fait sentir au niveau de l'activité de mise en marché de nouveaux produits. Ensuite, l'acquisition et l'allocation des équipements, des ressources humaines et des ressources financières s'exécutent à la hâte; ce qui entraine une insatisfaction permanente chez les dirigeants de 1 'entreprise.

Finalement, le contrôle des opérations est faible quant à la quantité et la qualité des informations qu'il engendre, d'où un processus décisionnel lent et complexe incapable de redresser la situation de l'entreprise. 


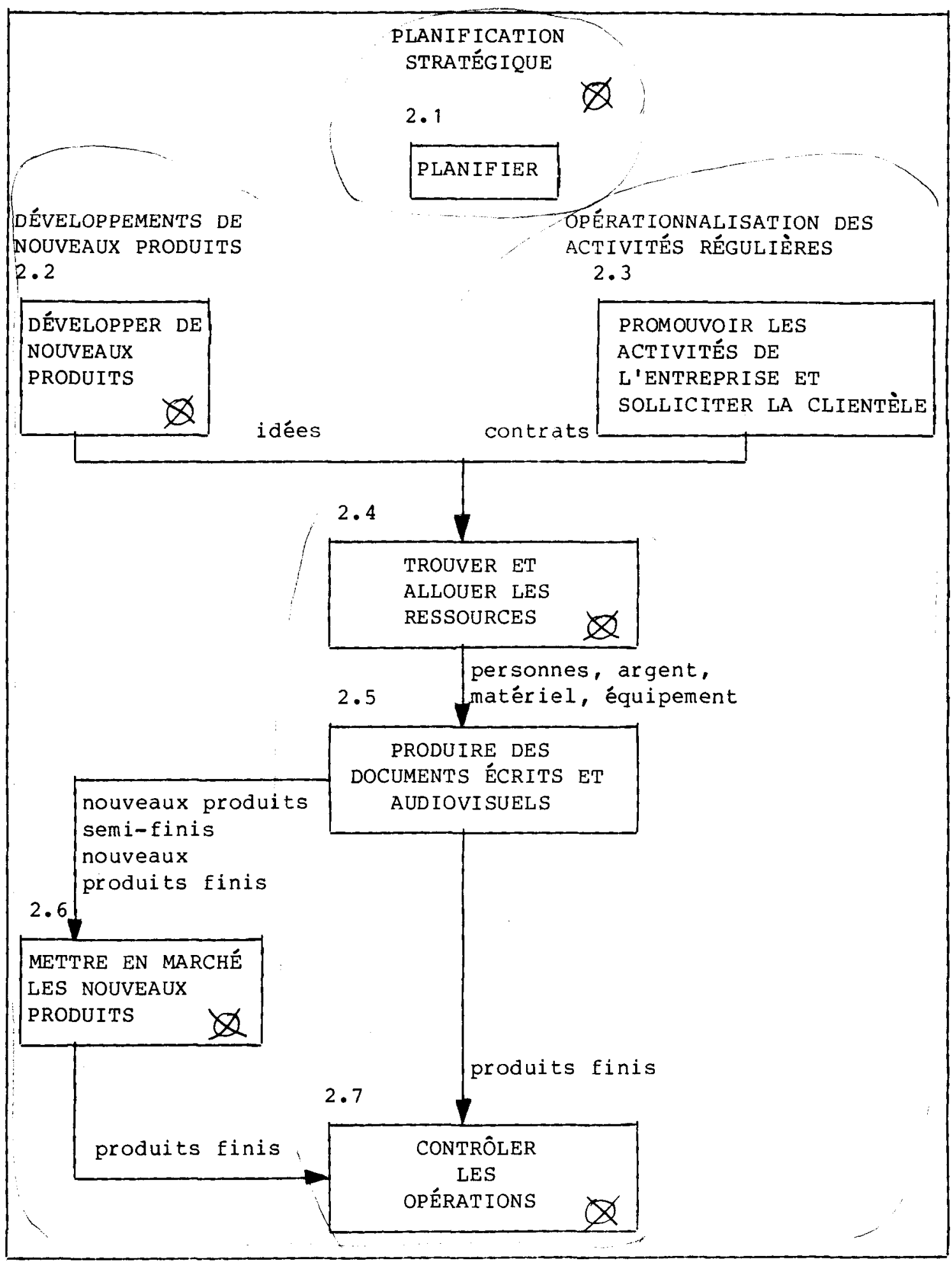

Figure 5.2

Système global 


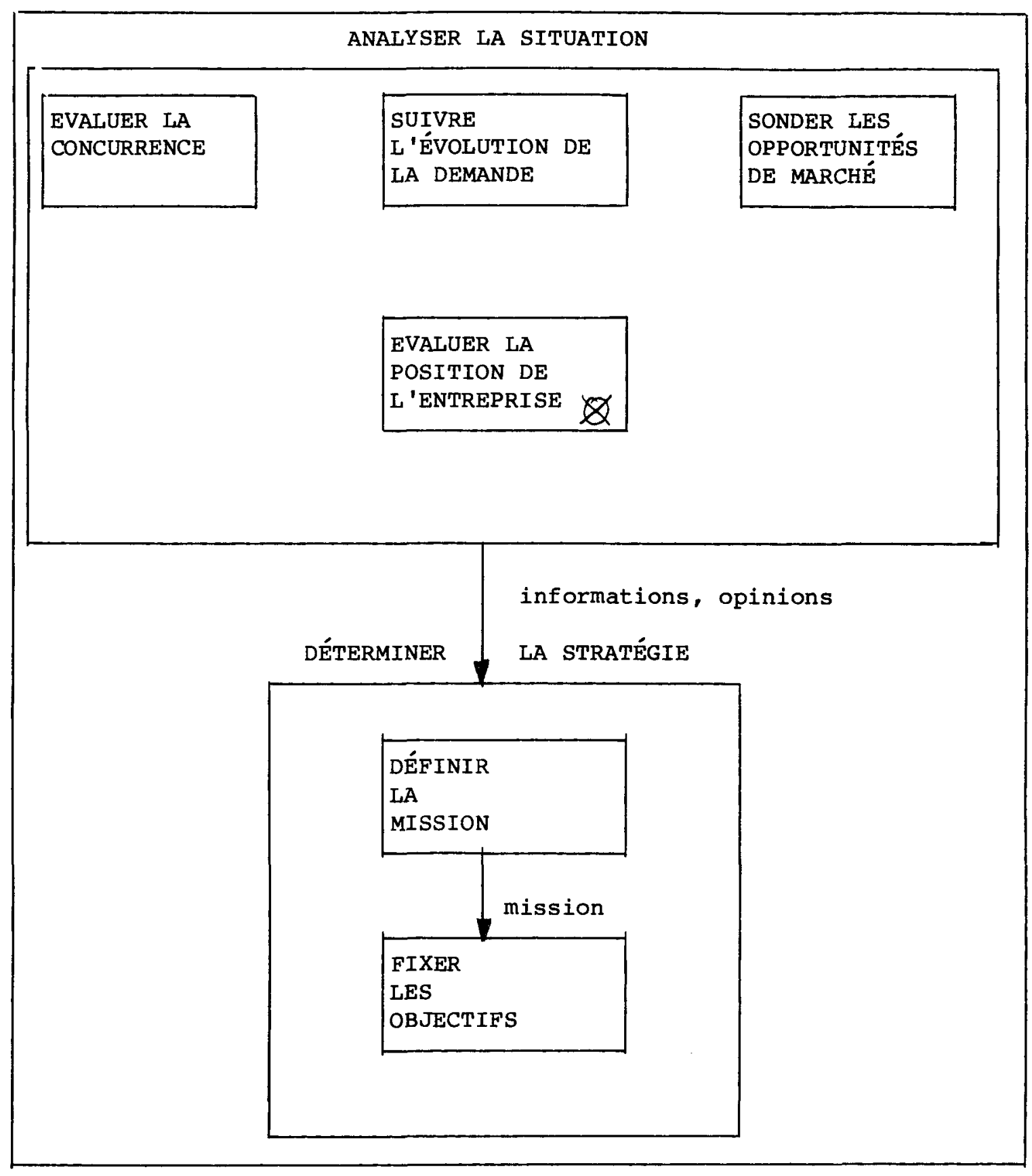

Figure 5.3

Sous-systène planification stratégique 

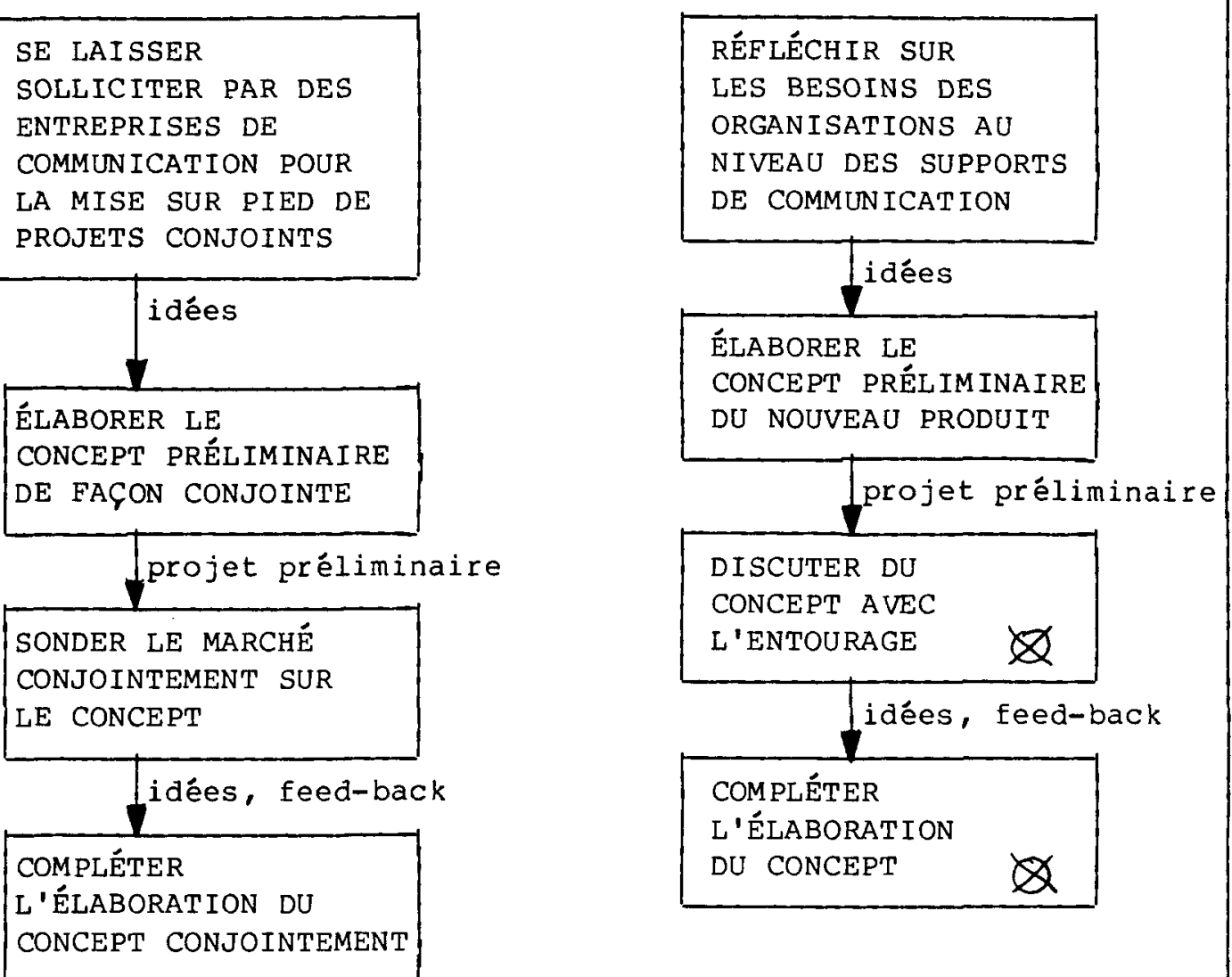

Figure 5.4 Sous-système développement 


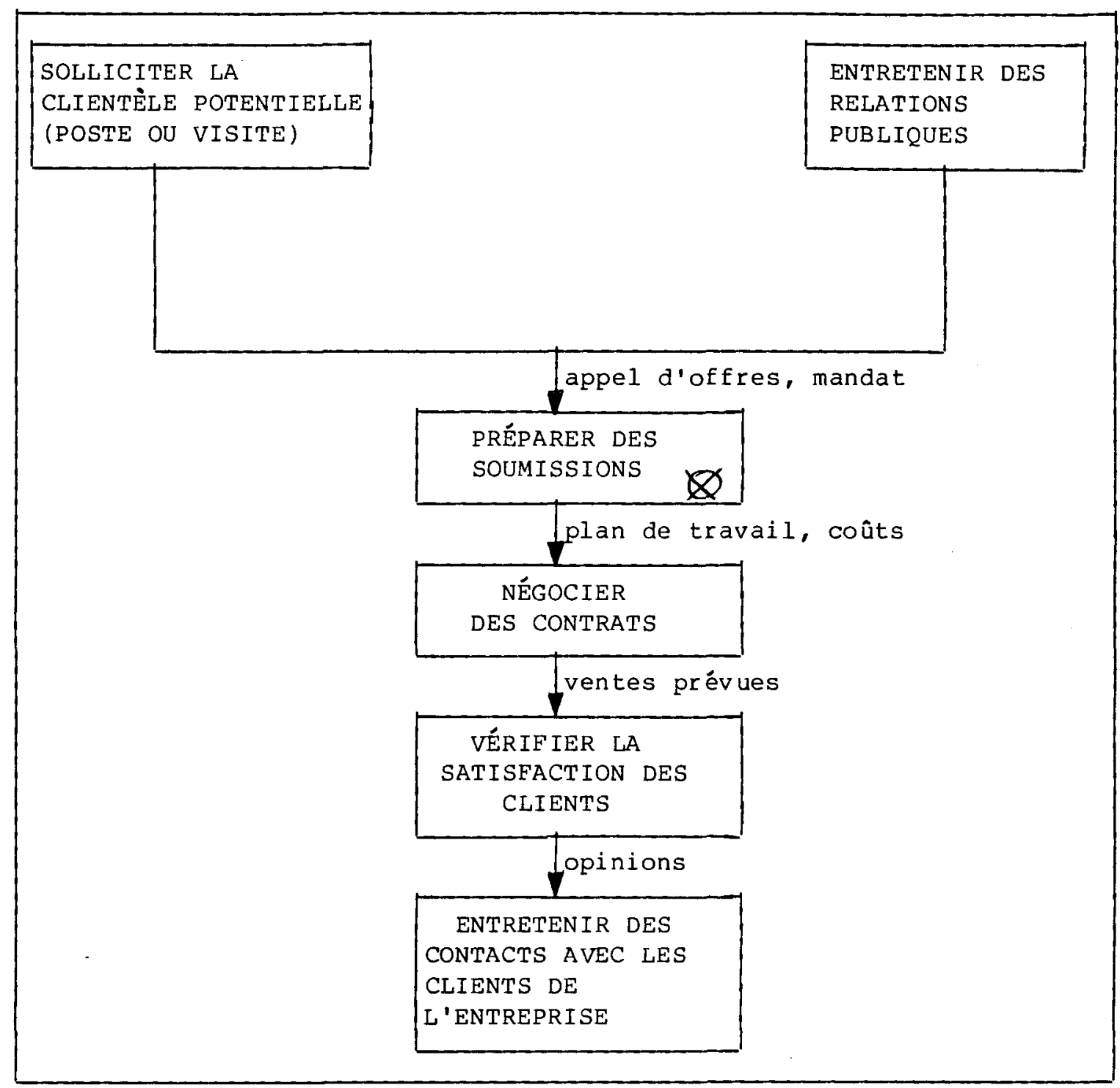

Figure 5.5

Sous-système promotion et vente 


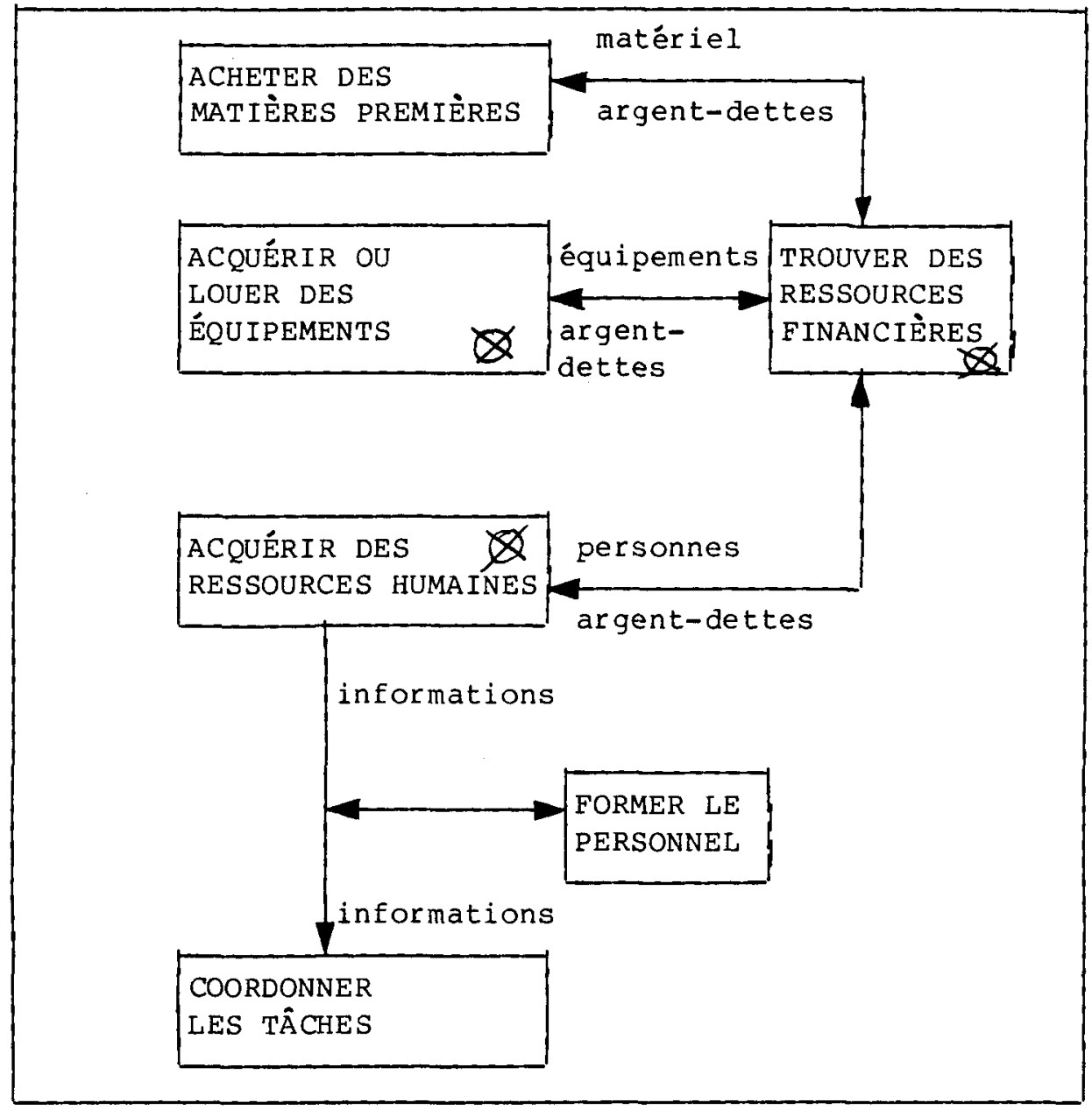

Figure $5.6 \quad$ Sous-système ressources 


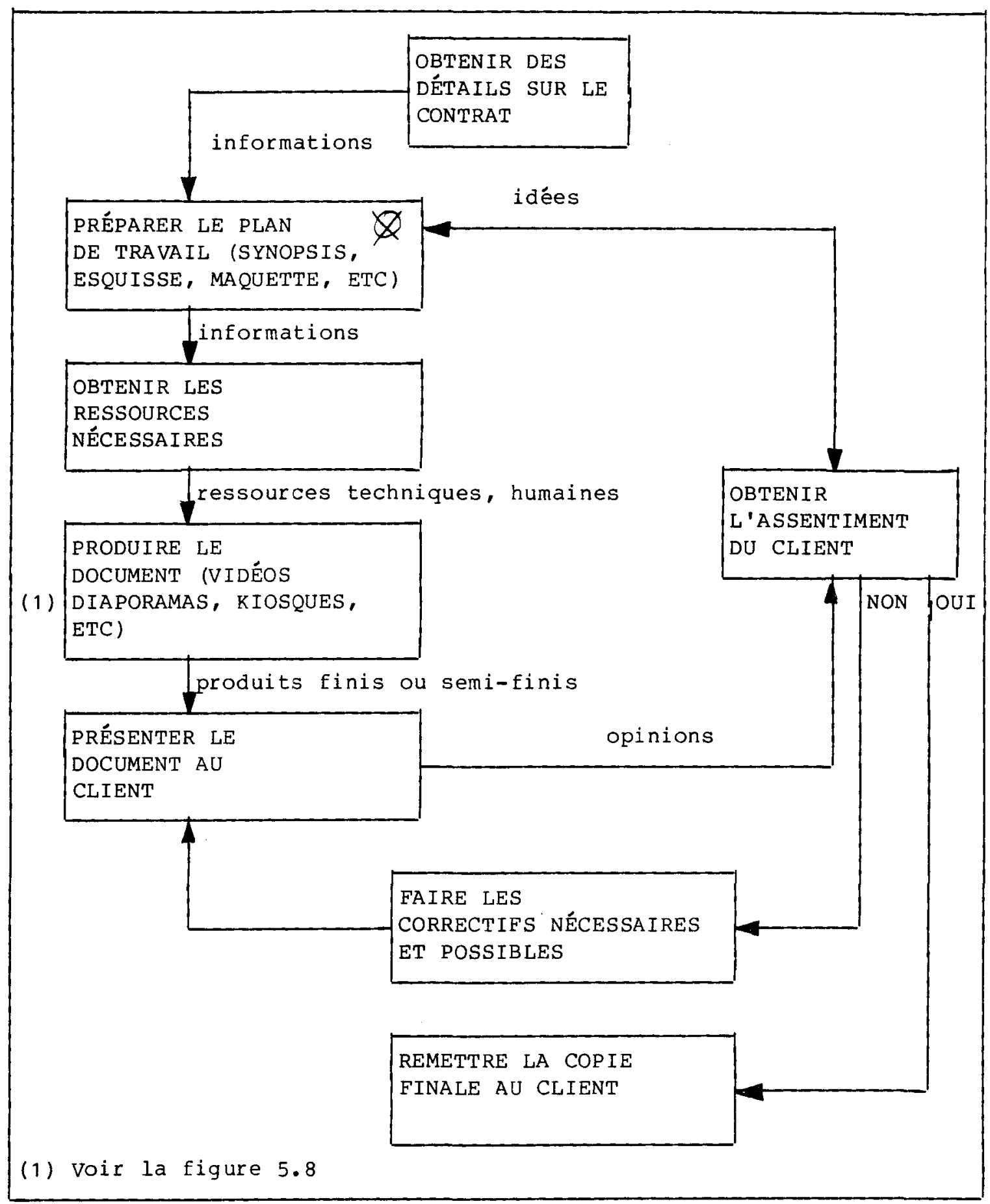

Figure $5.7 \quad$ Sous-système production 


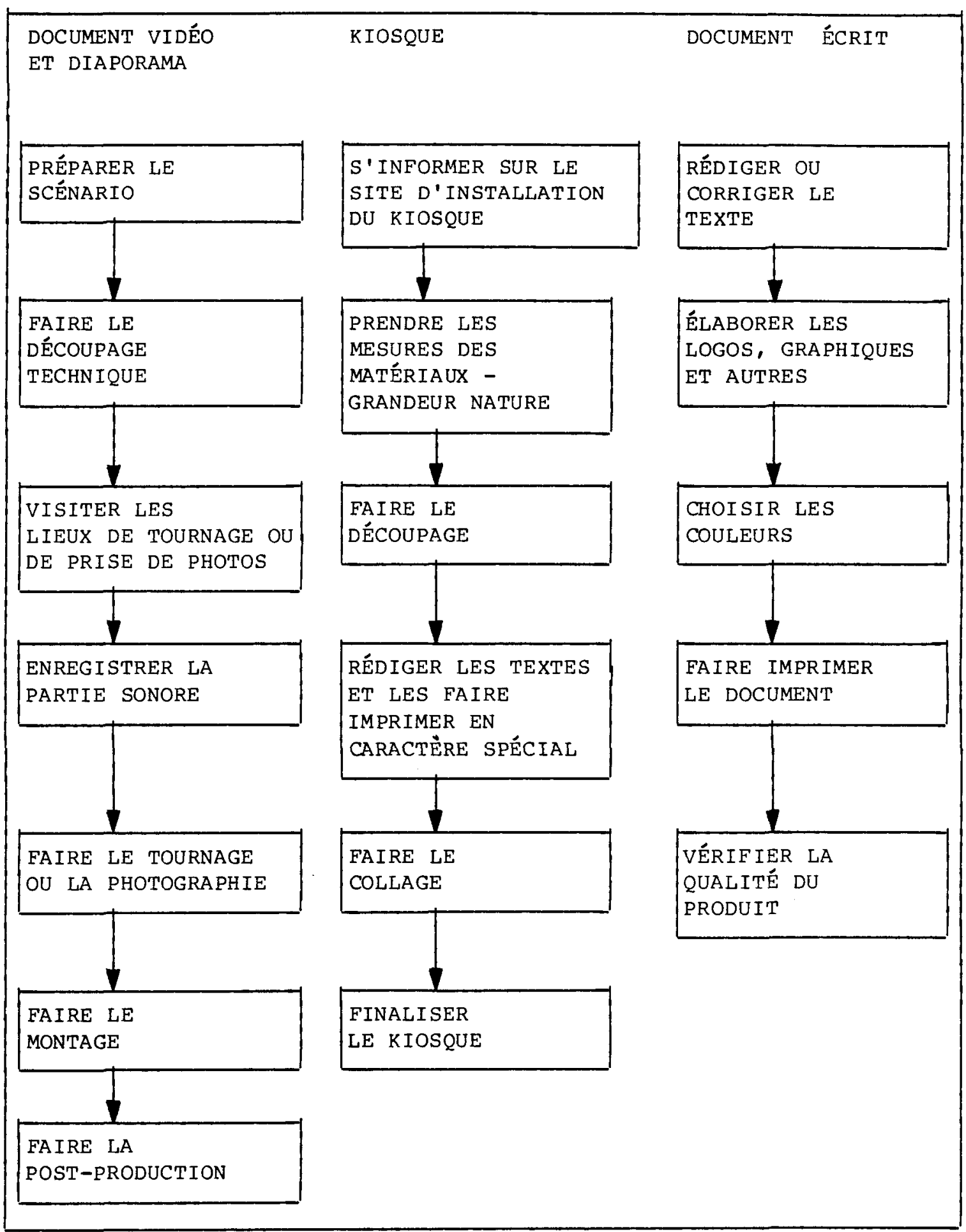

Figure 5.8 Sous-système production (suite) 


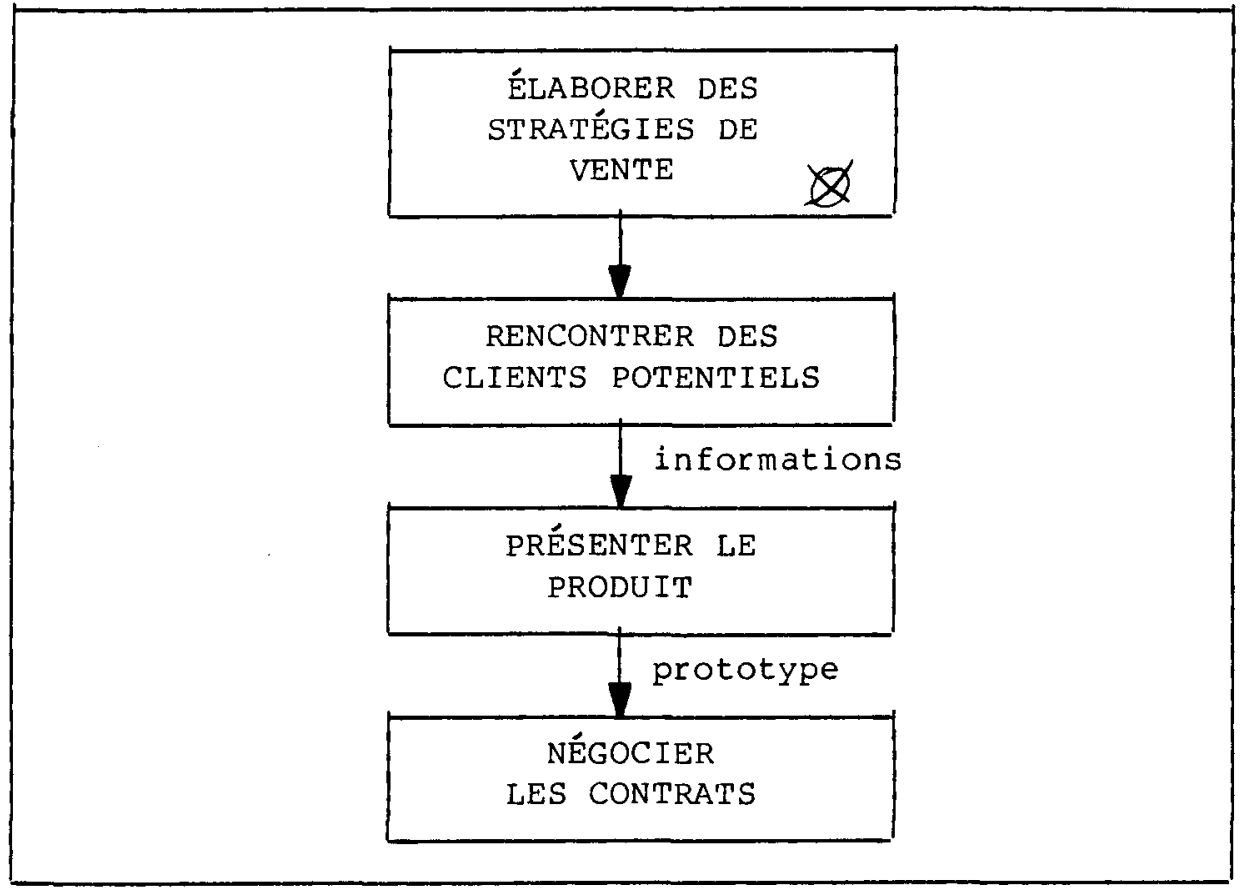

Figure 5.9

Sous-système mise en marché 


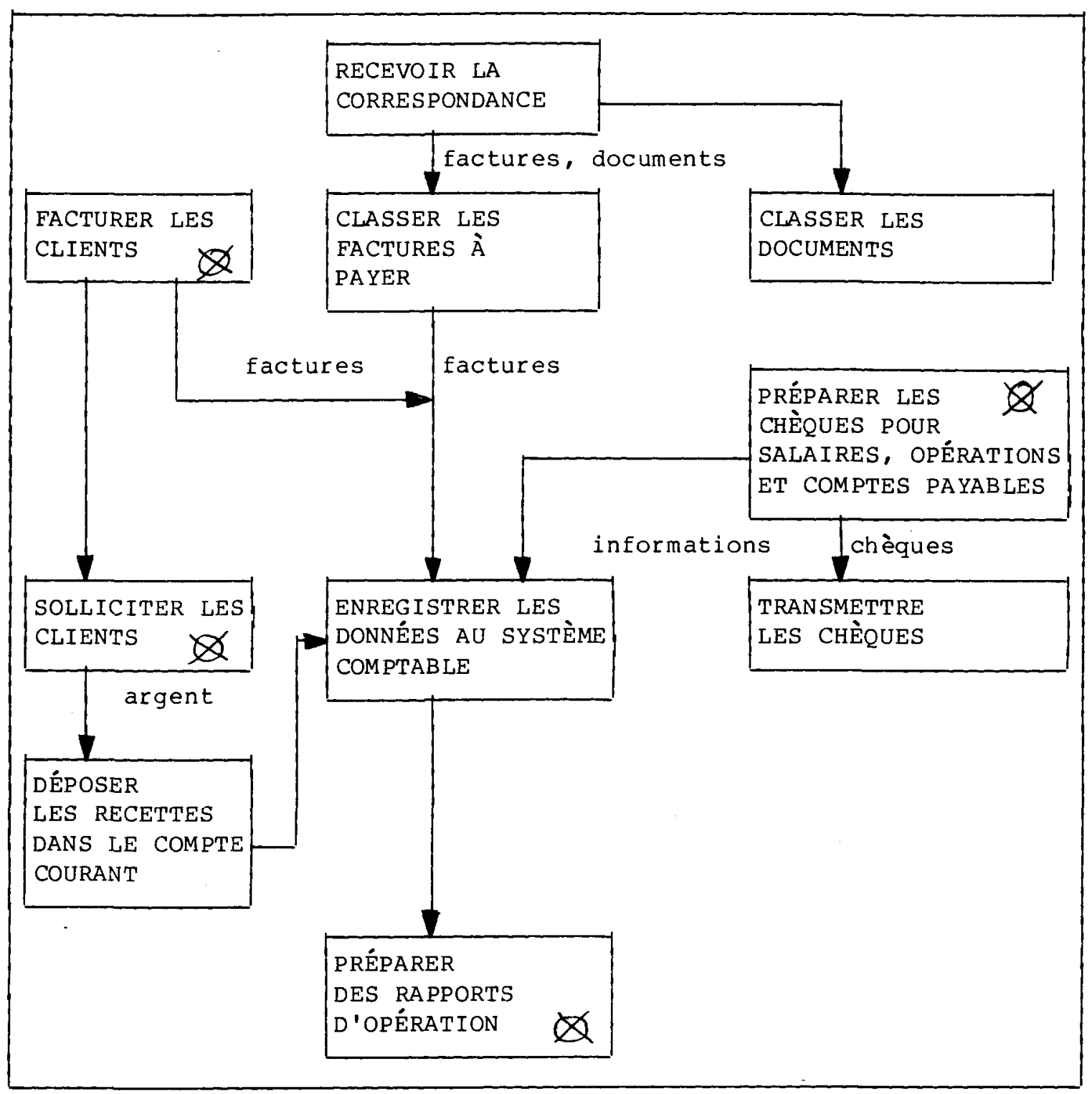

Figure 5.10

Sous-système contrôle 
V I

LE CLIMAT ORGANISATIONNEL 
L'objectif majeur de ce chapitre consiste à éclairer le lecteur sur une des caractéristiques les plus importantes de toute situation problématique: le climat organisationnel. Ainsi, nous nous attarderons à découvrir les attitudes et les comportements des dirigeants, le style de management et les relations de pouvoir dans l'entreprise. 
Le climat organisationnel "reflète les valeurs, les attitudes et les croyances des membres qui, à cause de leur nature, deviennent à leur tour des éléments du climat"(4).

Dès le début du présent diagnostic, nous avons pu constater dans l'entreprise, trois formes d'expression d'un climat organisationnel tendu. Au début, la communication entre les gestionnaires était au minimum. Ensuite, des manifestations d'agressivité verbale en relation avec des faits spécifiques se firent sentir pour finalement laisser place à des discussions plus ouvertes où on discute des frustrations et où on tente de mettre cartes sur table.

Nous dénotons aussi un certain découragement de la majorité des gestionnaires à travailler sans relâche, être peu payés et ne pas s'en sortir: on n'est pas très fier d'être propriétaire d'Imagem. on doute ausi de la satisfaction générale de la clientèle envers ses produits.

Le travail individualiste ou "cloisonné" qu'exerce chacun des gestionnaires, se réflète dans le climat organisationnel. En effet, les résultats visés par chacun ne rejoignent pas toujours la mission et les objectifs de l'entreprise, tels que définis lors du redressement du printemps 1987. Ces résultats servent plutôt les aspirations personnelles de chaque gestionnaire que le développement de l'entreprise. De façon 
imagée, on peut affirmer qu'ily a trois chefs mais pas d'indien dans l'entreprise. Suivant philippe Bernoux, ces buts "ne sont pas forcément opposés ou déviés par rapport à ceux des organisateurs même s'ils peuvent l'être. Simplement ils sont propres à chacun des acteurs... Chacun poursuit ses propres objectifs et l'organisation vit avec cette multiplicité plus ou moins antagoniste." (3)

On constate aussi qu'un des gestionnaires, M. Gendron, se retrouve un peu en marge des deux autres et ça peut s'expliquer en partie comme suit: premièrement, les gestionnaires M. Proulx et M. Brazeau éprouvent une certaine difficulté à faire un lien direct entre le temps passé à l'administration des affaires par M. Gendron et le peu d'information qu'ils en retirent. Deuxièmement, M. Gendron s'est trouvé un emploi à Montréal, qu'il intègre de façon graduelle depuis le 16 novembre 1987. Il se dit prêt à demeurer responsable de la comptabilité, à partir de Montréal. Depuis plusieurs mois, M. Gendron ne tenait pas compte de l'opinion de ses associés. Il passait deux à trois jours par semaine à Montréal pour faire des affaires et il avait embauché le personnel temporaire nécessaire pour l'appuyer dans ses tâches administratives. Finalement, la situation financière personnelle de chacun des gestionnaires affecte leurs rapports personnels en agissant sur leur vision de la situation actuelle. Plus les gestionnaires ont un budget personnel restreint, plus ils s'entendent pour dire que la situation actuelle de l'entreprise est 
critique à court terme. C'est le cas pour Messieurs Brazeau et Proulx. Moins la situation financière personnelle est difficile, moins la problématique est envisagée à court terme. C'est le cas de M. Gendron qui considère qu'on dispose du temps nécessaire au réajustement de la situation de l'entreprise à moyen terme. Ce qui confirme la réflexion de l'auteur Luc Brunet: "La réaction d'un individu à toute situation est toujours fonction de la perception qu'il en a. C'est la façon dont il voit les choses qui compte et non pas la réalité objective." (4)

Le pouvoir se définit comme "la capacité qu'a un individu ou un groupe d'obtenir que quelqu'un fasse ou pense quelque chose qu'il n'aurait ni fait ni pensé autrement" $(6)$. on ne peut donc sous-estimer son implication dans le climat organisationnel.

Chez Imagem, l'exercice du pouvoir se traduit de la façon suivante:

Bien que les trois gestionnaires tentent de conserver un certain niveau de pouvoir décisionnel par de nombreuses actions quotidiennes individualistes, voir même "indépendantistes", le pouvoir réel est entre les mains de Marc Gendron car il manipule une multitude d'informations. En discutant des sources du pouvoir,crozier et Friedberg avancent "qu'une 
source concrète du pouvoir dans les organisations réside dans la maitrise des relations avec l'environnement [...] Inutile d'insister sur l'importance des communications, sur le fait que l'information est du pouvoir parce qu'elle permet de mieux maitriser les incertitudes devant affecter l'organisation"( 3 ). Messieurs collerette et Delisle d'ajouter: "Ce n'est pas tant le fait de détenir telle information ou tel moyen de production qui permet à quelqu'un d'avoir du pouvoir dans une situation donnee mais bien le fait que cette information ou ce moyen de production soit valorisé par ceux que l'on veut influencer" ( 6$)$. Selon les circonstances, ces informations sont transmises oralement plutôt qu'avec documents à l'appui, partiellement ou en entier en fonction des contraintes de temps, selon sa propre analyse ou vision des choses, sans avoir eu recours à des conseillers du domaine. Ainsi, Marc contrôle l'embauche de personnel administratif, les stratégies de vente à privilégier, les coupures de budgets, la réorientation informelle de la mission de l'entreprise par les ventes qu'il choisit de faire. L'impuissance des deux autres gestionnaires Eace à cette situation les pousse à boycotter les productions non conformes à leur propre vision de la mission de l'entreprise, à faire leurs propres démarches pour trouver de nouveaux contrats et à réduire au minimum les informations qu'ils détiennent sur les activités de production. Selon philippe Bernoux: "Dans une organisation, tout acteur garde une possibilité de jeu autonome, qu'il utilise plus ou moins... si 
Ies acteurs sont autonomes, ils engagent leur autonomie dans les zones mal réglementées de l'organisation. Ces autonomies se combinent dans des jeux de pouvoir, le pouvoir central essayant de contrôler l'autonomie des acteurs qui, à leur tour, tentent de lui échapper"(3).

Il peut s'avérer rassurant pour les gestionnires d'identifier un responsable de la situation. Cependant, il faut tout de même se demander pourquoi personne n'est intervenu plus tôt. M. Gendron n'a pas nécessairement chercher le pouvoir; les circonstances l'y ont mené.

La collaboration de tous les gestionnires dans l'élaboration du diagnostic organisationnel constitue un autre fait important à souligner. Cela prouve bien leur volonté d'opérer un changement qui permettrait d'améliorer la situation actuelle de l'entreprise. 
Les tensions que nous avons relevées entre les gestionnaires de l'entreprise Imagem, permettent de conclure à un climat organisationnel relativement tendu. D'abord les circuits de communication tiennent le coup difficilement, puis le comportement des dirigeants en dit long; ils laissent paraitre leur découragement et leur manque de fierté d'y travailler. Finalement, on constate que le pouvoir est entre les mains de M. Gendron qui manipule la majorité de l'information et que messieurs Brazeau et proulx tentent de ramasser les miettes en contrôlant leurs productions. Cette situation déstabilisante gagne à être comprise par tous. 
106

VI I

LES INDICATEURS 


ce chapitre vise à poursuivre notre quête des
dysfonctionnements de l'entreprise, mais cette fois-ci à
partir de ses grandes fonctions.

Nous aborderons donc successivement la gestion du personnel, la gestion marketing, la gestion de la production et la gestion financière chez Imagem. Comme vous pourrez le constater, la fonction finance mérite une grande partie de notre attention dans la présente section. Cet effort se justifie par la situation financière précaire dans laquelle se retrouve l'entreprise depuis plusieurs mois. 


\section{PERSONNEL}

Au niveau du personnel, nous regroupons les indicateurs en sept thèmes distincts que voici:

\subsection{Expérience et compétence}

Les gestionnaires possèdent tous trois une formation en communications. Ils ont de plus acquis plusieurs années d'expérience en conception et production audiovisuelle. A ce jour, ils n'ont cependant développé que quelques projets isolés de graphisme, kiosques et photographie.

Un seul d'entre eux possède des notions de base en gestion des entreprises, sans toutefois être capable de les appliquer rigoureusement par manque d'expérience. (Par exemple, la gestion peu rigoureuse des comptes recevables et des comptes payables, la faiblesse de gestion des liquidités, les calculs adéquats du prix de revient, etc.).

Les forces de chacun des gestionnaires se dessinent de la façon suivante: 
Daniel Brazeau démontre des aptitudes à gérer les projets de production, à négocier les achats de matériel et à réaliser des productions audiovisuelles;

Marc Gendron démontre des aptitudes à solliciter les clients, entretenir des relations publiques avec l'extérieur et assurer le développement de nouveaux concepts dans l'entreprise;

Jean-Guy proulx démontre des aptitudes à solliciter les clients et entretenir des relations publiques avec l'extérieur; mais son implication sérieuse au niveau de la réalisation de documents audiovisuels et de la conception de nouveaux produits confirme ses forces dans le domaine.

Au sein des autres ressources humaines, certaines démontrent une autonomie et des capacités correspondant aux besoins de I'organisation. Que ce soit Charles Morin, technicien audiovisuel; constance Alain et Lucie vigneault, narrateurs et scénaristes; ou Denis Vachon, scénariste; ces gens dymaniques et compétents ont de fortes possibilités de faire partie de l'équipe d'Imagem de façon ponctuelle mais régulière selon le niveau des activités. 


\subsection{Répartition des tâches et responsabilités}

Les tâches ne sont pas définies de façon exhaustive (production de rapports financiers à date fixe, embauche de personnel, préparation de rapports de production sur projets spéciaux, opérations quotidiennes, etc).

Les tâches des pigistes sont assez bien définies et ceux-ci ont rarement eu des problèmes avec l'entreprise à ce sujet.

\subsection{Allocation des ressources humaines}

L'allocation des ressources humaines est souvent établie en fonction des disponibilités financières de chaque projet plutôt que des besoins des différentes productions.

1.4 Critères d'embauche et de sélection

La banque de ressources humaines de l'entreprise s'avère incomplète pour combler ses nombreux besoins (comédiens, secrétaires et autres).

L'embauche de pigistes et autre personnel temporaire s'effectue souvent à la dernière minute. 


\section{5 Négociation des contrats d'embauche}

Certains pigistes réalisent parfois des contrats avant même d'avoir négocié leur taux horaire ou leur cachet.

Le personnel temporaire et les nombreux pigistes ne reconnaissent pas toujours le même gestionnaire comme négociateur attitré pour les contrats. Ils négocient parfois leur mandat avec le responsable de la production et le cachet avec le responsable à 1'administration.

\subsection{Politiques salariales}

Les taux horaires et les cachets sont négociés à la $\mathrm{p}$ ì̀ce.

Certains pigistes se sentent actuellement trop payés. C'est le cas d'un rédacteur peu expérimenté pour lequel on verse des honoraires équivalents à un rédacteur expérimenté.

Certains honoraires de pigistes sont retenus dans les comptes payables lorsque le contrat pour lequel ces honoraires ont été engagés, demeure impayé à l'entreprise. Cette situation devient frustrante pour les pigistes qui n'en comprennent pas la logique. 


\title{
2. MARKETING
}

\author{
Au niveau du marketing, nous regroupons les indicateurs \\ en cinq thèmes:
}

\section{$\underline{2}$. 1 stratégies de vente}

Au cours de la dernière année, Imagem, par l'entremise de son responsable des ventes, a tenté d'élargir sa gamme de produits et services avec les kiosques, les pochettes d'information, la photographie, le vidéo disque, etc.

Le responsable des ventes éprouve des difficultés à refuser des projets même lorsqu'ils s'avèrent plus ou moins rentables. Il mise dans certains cas sur les retombées à plus long terme. De plus, sa principale préoccupation en terme de stratégie de vente consiste à faire augmenter les entrées d'argent dans l'entreprise pour combler les déficits bancaires quotidiens.

Ces deux positions ne sont toutefois pas endossées par les deux autres gestionnaires puisqu'elles vont, selon eux, à l'encontre de la mission de l'entreprise telle que définie lors du redressement du printemps 1987. 


\section{2 outils de promotion}

Le vidéo promotionnel destiné à la sollicitation de la clientèle est réalisé avec un minimum de moyen dans loptique de demontrer au client les productions de l'entreprise. Il ne révèle cependant pas toutes les possibilités et capacités de l'entreprise. Un tel outil promotionnel pourrait nécessiter des investissements de près de $\$ 5000$

Actuellement, et pour les mois à venir, on peut affirmer que le contrat négocié à l'été 1987 avec les productions 24-30, pour la série vidéotour, représente la vache à lait chez Imagem. On constate aussi que, mis à part ce projet, les ventes prévues pour le prochain semestre à partir des contacts des derniers mois, sont très intéressantes. On trouve le détail de ces prévisions au tableau 7.1. De plus, Imagem a fait la conception d'un projet intéressant d'auto-construction qui pourrait représenter un chiffre d'affaires substantiel, si on ne répète pas les mêmes erreurs de mise en marché qu'avec opton. Les revenus que ce projet peut engendrer ne sont pas inclus dans le tableau 7.1. L'auto-construction est un document audiovisuel, appuyé par une documentation écrite, qui permettra à un néophyte de la construction domiciliaire, d'agir à titre de sous-contractant sur sa propre maison, à partir de l'avis d'experts qui participent au projet d'auto- 
construction. Les documents écrits pourraient même contenir des coupons-rabais très intéressants pour le nouveau propriétaire domiciliaire.

Tableau 7.1 Ventes potentielles d'Imagem

\begin{tabular}{|c|c|c|c|}
\hline \multirow{3}{*}{ NOM DU } & \multirow[b]{2}{*}{ CATÉGORIE } & \multicolumn{2}{|c|}{ VENTES } \\
\hline & & SEMESTR IELLES & ANNUELLES \\
\hline & $\begin{array}{ll}D E & R E V E N U S \\
\end{array}$ & $\begin{array}{l}(\text { nov. } 87 \quad \grave{a} \\
\text { avril } 88)\end{array}$ & $\begin{array}{l}(\text { nov. } 87 \\
\text { oct. } 88)\end{array}$ \\
\hline Relance & vidéo & $10000 \$$ & $10000 \$$ \\
\hline vidéotour & vidéo & 72000 & 72000 \\
\hline $\begin{array}{l}\text { Projet PME- } \\
\text { municipalités }\end{array}$ & vidéo & - & 20000 \\
\hline $\begin{array}{l}\text { Place du } \\
\text { centre }\end{array}$ & vidéo & 10000 & 10000 \\
\hline $\begin{array}{l}\text { Parc de la } \\
\text { Gatineau }\end{array}$ & diaporama & 5000 & 5000 \\
\hline $\begin{array}{l}\text { Ville de } \\
\text { Gatineau }\end{array}$ & vidéo & 30000 & 30000 \\
\hline AEEFO & vidéo & 15000 & 15000 \\
\hline Autres & $?$ & $\frac{?}{142000 \$}$ & $\frac{95600}{237600 \$}$ \\
\hline
\end{tabular}




\section{PRODUCTION}

Au niveau de la production, nous regroupons les indicateurs en cinq thèmes.

\subsection{Gestion des projets de production}

personne dans l'entreprise, n'a l'entière responsabilité de l'ensemble des productions vidéo, diaporamas, kiosques ou autres. Messieurs Brazeau et proulx dirigent à tour de rôle, en fonction de leur disponibilité et expérience, les projets qui leur sont soumis.

on ne prépare aucun calendrier des activités. Tous les engagements s'établissent de façon orale et il arrive souvent que les productions ayant un faible intérêt pour les gens de la production, soient reportées à plus tard. Les horaires de travail "oraux" sont aussi réaménagés régulièrement et désorganisent l'horaire de travail des uns et des autres à tour de rôle.

Les synopsis des cinq premiers vidéos du projet Vidéo Tour (sur un total de douze), ont été remis au 
client deux à trois semaines après la date prévue. Les productions à venir ne devraient pas connaître les mêmes problèmes de délais, aux dires des responsables de la production.

La production de vidéos, diaporamas, kiosques et photographies est souvent reportée pour cause de manque de matériel, de non-disponibilité des pigistes, d'étapes préliminaires incomplètes, etc.

\subsection{Contrôle de qualité}

Les gestionnaires sont fiers des productions suivantes: le Lac des Fées (vidéo); la ville de Gatineau (vidéo); $C K C H$ (message publicitaire pour la télévision); l'Association des enseignants et enseignantes franco-ontarien (vidéo d'information); Gendron Lefebvre (vidéo) et pole Lite (kiosque). par contre, ils sont déçus de plusieurs petites productions vidéo, du diaporama de voyage El-Air, etc. 


\subsection{Ressources techniques disponibles}

Plusieurs équipements détenus par Imagem restent inutilisés depuis plusieurs mois. on se souviendra que la majorité des équipements encore détenus par l'entreprise avaient été achetés pour offrir les services de diffusion opton et faire la conception et la production des documents visuels de nature industrielle. Ils représentent une valeur marchande de $\$ 8500$. Vous trouverez la liste de ces équipements à l'annexe 1 .

Les équipements actuellement détenus par Imagem ne permettent donc pas de réaliser dans l'entreprise, l'ensemble des activités de production vidéo de qualité "broadcast". Alors qu'ils permettent de faire le montage préliminaire; le tournage et la post-production ne sont pas possibles avec ces équipements. On loue ces services dans des studios spécialisés dans la catégorie "broadcast". On ne peut pas penser acquérir ces équipements au stade actuel car les activités de l'entreprise ne permettent pas le remboursement d'une telle dépense en capital. 


\subsection{Ressources humaines}

on réunit rarement des équipes de "brainstorming" pour trouver des idées originales et nombreuses sur une même production.

La majorité des gestionnaires se sentent à l'aise dans la production vidéo et certains services techniques, mais le sont plus ou moins dans la production de kiosques, de photos et de diaporamas.

\section{FINANCE}

Notre analyse de la situation financière de l'entreprise porte sur les opérations jusqu'en date du 31 octobre 1987 . Les divers rapports financiers et annexes qui sont la source de cette analyse (soit le bilan, l'état des résultats, la liste des comptes à recevoir, la liste des comptes à payer, etc.) n'avaient pas été mis à jour depuis au moins 6 mois. on dispose depuis plus d'un an des équipements nécessaires à la mise à jour des finances mais les ressources humaines manquent d'expérience. Notre soutien technique en terme de comptabilité, s'est donc avéré nécessaire pour obtenir des informations récentes. Vous trouverez les états financiers comparatifs aux annexes 2,3 et 4 . Nous avons regroupé les indicateurs financiers sous six thèmes distincts. 


\subsection{Analyse des états financiers}

Cette analyse portera sur les données comparatives entre le rapport issu du redressement de mars 1987, le rapport du 30 juin 1987 et celui du 31 octobre 1987 .

Le montant des pertes d'opérations obtenues à chacune des trois périodes de référence diminue progressivement, lorsque nous le comparons sur une base mensuelle: au 29 février 1987 , $\$ 3145 ;$ au 30 juin 1987, \$926; au 31 octobre 1987, \$245. On peut donc conclure que les revenus de production couvrent de plus en plus les frais variables et les frais fixes mensuels.

Le chiffre d'affaires des trois périodes de référence, lorsque ramené sur une période d'un mois pour fins de comparaison, est le suivant: au 29 février $1987, \$ 10276$ par mois; au 30 juin 1987, $\$ 11694$ par mois; et au 31 octobre $1987, \$ 13630$.

Les frais de sous-traitance sont de loin, les coûts variables les plus importants chez Imagem. En pourcentage des ventes, ils représentent 278 au 28 février $1987 ; 238$ au 30 juin $1987,25 \%$ au 31 octobre 1987. 
Le ratio d'endettement (le rapport entre le total du passif et le total de l'actif) passe de 1098 au 28 février 1987, à 898 au 30 juin 1987 et $77 \%$ au 31 octobre 1987.

Au 31 octobre 1987, l'emprunt à long terme équivaut à environ $68 \%$ de la valeur nette des immobilisations aux livres.

A la même date, la marge de crédit est utilisée à $85 \%$ de sa capacité, soit $\$ 5500$ environ.

\subsection{Analyse du prix de revient des produits}

Les principaux commentaires que nous pouvons faire en analysant le tableau comparatif de vingt produits distincts présentés aux annexes 5 et 6 sont les suivants:

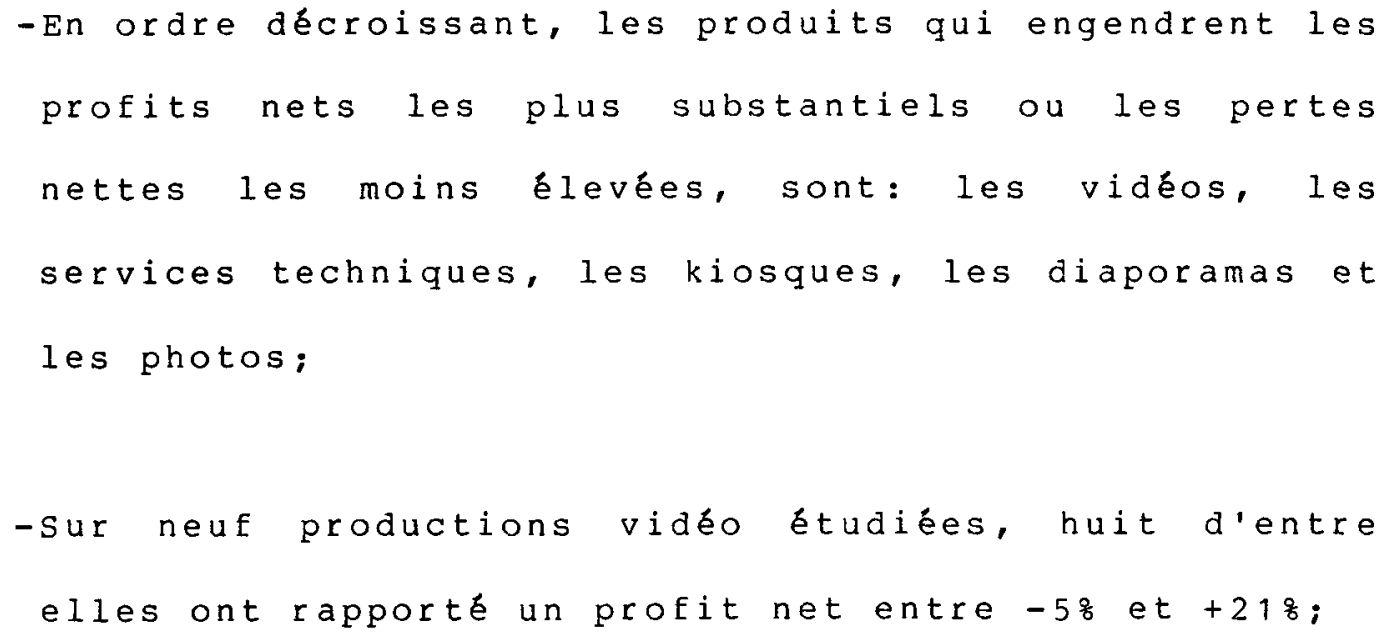




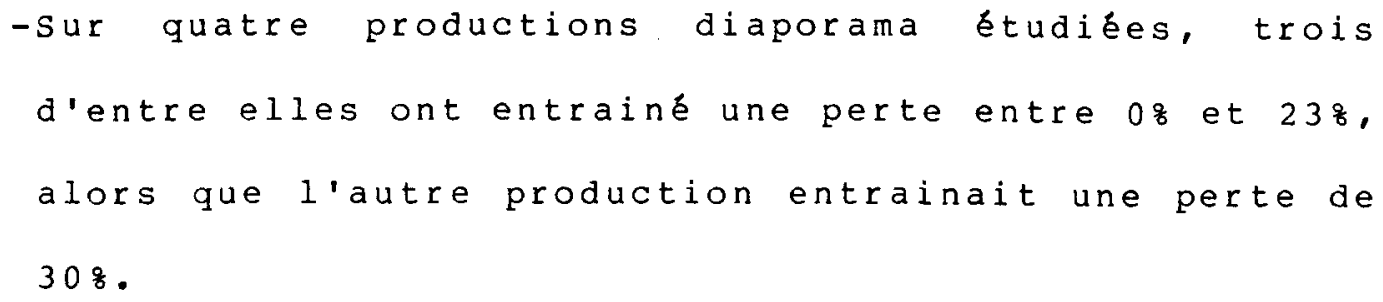

4.3 Calcul du seuil de rentabilité annuel

Le calcul du seuil de rentabilité apporte des précisions interessantes. Ainsi on constate que l'entreprise Imagem, dans ses conditions actuelles d'opération, doit atteindre un chiffre d'affaires annuel d'environ $\$ 192000$ pour rencontrer ses coûts variables et ses coûts fixes.

Coûts fixes annuels:

Coûts variables annuels:
$96000 \$$

$50 \%$ des ventes

D'où le calcul suivant du seuil de rentabilité annuel:

$$
\text { seuil de rentabilité= }
$$$$
\text { 모믐ㅁㅁㅇㅡ }=\$ 192000
$$ 


\subsection{Gestion des comptes à recevoir}

L'analyse de la liste des comptes à recevoir au 30 juin et au 31 octobre 1987, permet de dégager les éléments qui suivent:

Au 30 juin $1987,98 \%$ des comptes à recevoir étaient dûs depuis moins de 30 jours. Il n'en est pas de même pour les comptes à recevoir en date du 31 octobre 1987, où seulement $54 \%$ sont dûs depuis moins de 30 jours, $27 \%$ entre 30 et 60 jours et $19 \%$ depuis 60

jours ou plus. on constate alors un certain relâchement dans la perception des comptes à recevoir, qui influence négativement les liquidités de l'entreprise.

Parmi les comptes à recevoir au 31 octobre 1987, plus de 50\% sont dûs par l'entreprise production 24-30, pour le projet vidétour. La principale cause de cet etat de chose correspond au retard indu dans la preparation des rapports de production. En date du 16 novembre 1987, seulement deux rapports de production ont été expédiés au client sur une possibilité de quatre. 


\subsection{Gestion des comptes à payer}

L'analyse de la liste des comptes à payer au 30 juin et au 31 octobre 1987, permet de dégager les eléments suivants:

Au 30 juin 1987, 238 des comptes à payer représentaient des dépenses courantes; 22 étaient dûs entre 0 et 30 jours; 208 entre 30 et 60 jours; et $35 \%$ depuis 60 jours ou plus. Il est indéniable que bon nombre de fournisseurs se sentent lésés, bien que cette situation ameliore les liquidites bancaires d'Imagem, du moins en apparence;

Au 31 octobre 1987 , le portrait de la situation s'est amélioré au niveau de la gestion des comptes payables. La répartition âgée est de 9 응 courant, $69 \%$ entre 0 et 30 jours; 48 entre 30 et 60 jours et $18 \%$ de 60 jours ou plus;

Les sommes les plus importantes sont dûs à production Marc, Le Droit, Monique Piché, Action Vidéo, Gerard Parizeau et sound Venture productions;

Les retards dans le paiement des comptes fournisseurs compliquent la tâche du principal acheteur de I'entreprise. C'est pourquoi Daniel Brazeau demande 
souvent au service de l'administration, de payer régulièrement les fournissseurs suivants: productions Marc, United Video et Baldwin Audio-visual avec qui il transige régulièrement.

\subsection{Autres exigibilités à court terme}

Comme il a déjà eté expliqué dans l'historique de l'entreprise, au 29 février 1987, plusieurs crénciers voulaient récupérer leur dû. Ce sont surtout les représentants des déductions à la source des deux paliers gouvernementaux et de la taxe de vente provinciale, qui ont démontré le plus d'intransigeance. Finalement, une entente de remboursement s'échelonnant entre six et douze mois a été conclue.

\footnotetext{
C'est ainsi que le solde est passé de près de 12000 s en février 1987 à près de $4000 \$$ au 31 octobre 1987. Un - revirement très significatif.
}

\subsection{Disponibilités financières à venir, à court terme}

Une somme totale de près de $22000 \$$ sera récuperrée par la vente d'équipements inutiles et la seconde tranche de I'indemnisation de l'assurance-feu. 
Les actuels gestionnaires de l'entreprise ne disposent pas de liquidités permettant de renflouer les coffres de l'entreprise.

Une subvention à la formation en entreprise pourrait être consentie par la commision de Formation professionnelle, mais seulement au printemps 1988.

\section{AUTRES INDICATEURS}

\section{1 operations quotidiennes}

En date du 15 octobre 1987, à 2 semaines de délai d'un déménagement rendu nécessaire par l'expiration d'un bail de quatre mois, on ne savait pas encore où s'installer temporairement. Leur ancien local, ne sera pas prêt avant décembre 1987 .

\section{$5 \cdot 2$ système d'information}

L'information comptable est très peu transmise, que ce soit: les états financiers, les liquidités disponibles, la liste des comptes à recevoir, la liste des comptes à payer, les budgets de caisse et autres. En date du 5 novembre 1987, les deux 
gestionnaires non affectés à l'administration
réteraient leur demande d'information à ce niveau.

Le système comptable révèle plusieurs irrégularités: L'enregistrement des revenus se fait sur une base de prix de revient plutôt que sur la base des différentes catégories de revenus. Ainsi, le système comptable ne peut pas nous indiquer si l'entreprise a vendu plus de diaporamas que de vidéos, plus de kiosques que de services d'installation technique, etc;

La préparation des rapports de production spéciaux tels les productions 24-30, est rendue difficile entre autres par la perte temporaire de certaines pièces justificatives (factures, transferts de droits de diffusions, etc.).

Un seul des trois gestionnaires, le responsable de l'administration, se sent à l'aise avec l'ensemble du processus de contrôle des donnes comptables (faire une facture, imprimer un chèque, questionner le système comptable, etc.). Les deux autres gestionnaires craignent ainsi deéprouver certaines difficultés à prendre la relève des activités administratives, advenant le départ précipité du responsable actuel. 
5.3 Respect de la mission et des objectifs issus du redressement

En se referant à la description de la mission et des objectifs tels que présentés au chapitre II, nous constatons à ce stade-ci du diagnostic que ceux-ci n'ont pas été atteints à plusieurs points de vue:

En plus des produits video et diaporama ainsi que la
location d'equipements, on a elargi la gamme de
produits et services aux kiosques et aux prises de
photos qui n'etaient pas prévus dans la mission
initiale.

La réduction des frais d'operation a été faite partiellement en négligeant les coûts reliés aux équipements conservés inutilement par l'entreprise.

Les outils de gestion et d'information ont été ameliorés mais pas suffisamment pour répondre aux besoins criants des gestionnaires. Ex: 1iquidités, budgets de caisse, états financiers, liste des comptes payables et recevables, etc. 
La difficulté de l'exercice que nous venons de faire ne réside pas dans l'énumération, mais plutôt dans la sélection des indicateurs pertinents.

Ainsi, au niveau des ressources humaines, ce qui retient le plus notre attention; c'est leur forte concentration dans les activités de promotion et de production, contrairement aux activites de gestion.

D'autre part, nous sommes surpris que le plan marketing corresponde peu à la stratégie et à la mission de l'entreprise. Pourtant, un bref survol des contrats potentiels de la prochaine anné laisse entrevoir un défi intéressant.

Au niveau de la production, nous constatons que la gestion des projets n'est pas bien structure. De plus, certains equipements actuellement détenus par l'entreprise ne correspondent que partiellement à ses besoins pour les productions de type "broadcast".

Finalement, au niveau financier, nous dénotons une constante amelioration depuis le printemps 1987. Entre autres, signalons que les pertes d'operation mensuelles se situaient à $\$ 245$ en octobre, comparativement à $\$ 3145$ en février. Le chiffre d'affaires se rapproche de plus en plus du seuil de rentabilité. Le fonds de roulement s'améliore considerrablement 
et lentreprise pourra disposer d'ici quelques mois, d'une somme de près de $\$ 22000$ par la vente d'equipements désuets et l'encaissement du solde résiduel de l'assurance-feu. 
V I I I

LA PROBLEMATIQUE DE L'ENTREPRISE IMAGEM 
Le present chapitre vise d'abord à effectuer un rappel des differents problèmes soulevés au cours du diagnostic. N'oublions pas que ces problèmes ne contribuent pas tous à un même degré, à maintenir la situation précaire dans laquelle se trouve actuellement l'entreprise Imagem.

Une selection des problèmes majeurs sera ensuite effectuée à l'intérieur de cette énumération. cette sélection servira à définir le point d'ancrage.

Une analyse sérieuse de la situation d'une entreprise ne met pas le chercheur à l'abri des bouleversements de dernière minute. Ainsi, il peut s'avérer nécessaire de solutionner des problèmes ayant des retombées à très court terme dans un premier temps, pour devenir apte ulterieurement à passer aux problèmes de fond.

L'énoncé de la solution retenue et la définition du système d'activités pertinent viendront compléter l'étude de la problématique de l'entreprise Imagem. 
1. CANDIDATS AU POSTE DE PROBLEME

\subsection{Planification stratégique}

- I'inexistence de mission et stratégies communes pour tous les gestionnaires;

- le manque de cohérence entre les activités de mise en oe uvre stratégique et la mission définie lors du redressement du printemps 1987 (par exemple, le plan marketing implicite);

-un certain degré d'indépendance entre les gestionnaires, encore plus marqué entre plusieurs systèmes d'activités humaines;

-dans l'environnement outaouais, un mince développement de l'image de marque de l'entreprise; que ses propriétaires aimeraient bien voir diffusée;

-une certaine inéquation entre les ressources dont l'entreprise dispose, ce que les propriétaires aimeraient faire et le marché actuel;

-une faiblesse marquée dans la planification stratégique à court, moyen et long terme. 
1.2 Développement de nouveaux produits

- le manque de ressources humaines pour les analyses de marché, de faisabilité et l'élaboration des stratégies de mise en marché;

- le manque de ressources financières pour soutenir le développement de nouveaux produits à tous les points de vue;

\subsection{Opérationnalisation}

Sous-système promotion et vente

- la difficulté de négocier avec les clients un prix qui assure une marge bénéficiaire nette suffisante, tout en offrant un produit de qualité;

Sous-système allocation des ressources

- une banque de ressources humaines insuffisante pour combler les nombreuses activités de production;

-un manque de ressources humaines de gestion; 
- I'inexistence d'un processus d'embauche rigoureux et de politiques salariales;

- la désuétude d'une partie importante des équipements de l'entreprise: environ trente pour cent (308);

\section{Sous-système production}

- le manque de consultation dans le processus de production;

- le manque de coordination dans la gestion des différents projets;

Sous-système contrôle

-l'utilisation d'un système comptable informatisé partiellement intégré;

- la faible diffusion de l'information comptable;

- la facturation tardive des projets;

- le manque de contrôle du prix de revient des produits; 
-l'inexistence de politiques de comptes à recevoir et de comptes à payer;

- le départ précipité de monsieur Gendron, responsable des ventes et de l'administration dans l'entreprise.

\section{CHOIX ET JUSTIFICATION DU POINT D'ANCRAGE}

Des discussions entre les intervenants les amènent à s'attarder d'abord à leur système de planification stratégique. Ce choix ne se fait pas sans difficulté, mais il semble le mieux adapté à une vision globale de la situation. En fait, trois éléments conditionnent ce choix: le contexte actuel, la philosophie de l'entreprise et la personnalité des propriétaires.

Premièrement, le contexte actuel se caractérise par deux faits importants: d'une part, la situation financière de l'entreprise (quoique irrégulière) n'est plus précaire suite à la perception de l'assurance et à l'accroissement du chiffre d'affaires des derniers mois. D'autre part, les contrats prévus pour les six mois à venir garantissent une base non négligeable de rentrées de fonds.

\footnotetext{
Deuxièmement, la philosophie de l'entreprise laisse croire que l'on ne veut surtout pas "y faire beaucoup de
} 
choses" mais qu'on veut $y$ faire "les bonnes choses". Ce revirement de la situation permettrait d'améliorer l'image de I'entreprise dans l'environnement.

Troisièmement, la personnalité des propriétaires permet de constater qu'ils aimeraient bien passer d'une situation d'opérateurs à une situation de décideurs; sentir qu'ils ont quelque chose à voir avec le développement de leur entreprise.

Finalement, ajoutons que ce que les intervenants qualifiaient dans un premier temps de système de planification stratégique, s'est avéré mieux servi par un système de management stratégique à la lumière d'une revue de littérature adéquate. Les auteurs Bamberger et Chicha ont grandement éclairé notre recherche d'un système d'activité pertinent à la problématique de l'entreprise.

Suite à ces considérations, nous proposons donc comme premier niveau de problème à solutionner:

le réalignement stratégique et les réajustements de mise en oeuvre stratégique.

L'image riche de l'entreprise Imagem nous a donc permis d'identifier la principale zone problématique que représente le réalignement stratégique. Cependant le départ précipité de 
Marc Gendron, qui contrôlait jusqu'à ce jour une grande partie de l'information, nous a obligés en tant qu'intervenant, à concentrer nos efforts pour quelques semaines sur le transfert des informations et de certaines activités de contrôle aux deux gestionnaires en place. Ces efforts se sont concentrés plus particulièrement sur notre soutien administratif dans:

- l'évaluation hebdomadaire de la situation financière de l'entreprise par la préparation de budgets de caisse ;

- l'élaboration d'un plan d'action à très court terme pour accélérer les rentrées de fonds dans l'entreprise (perception des comptes à recevoir, préparation de rapports de production, facturation rapide des contrats, etc);

- l'évaluation des créditeurs et leur échéancier de remboursement;

$\grave{A}$ partir de la deuxième semaine de décembre, les efforts de tous les acteurs se sont enfin tournés vers l'élaboration de solutions qui permettront à l'entreprise d'engendrer des actions concrètes et cohérentes pour assurer sa survie à moyen et long terme. 
3. DÉFINITION DU POINT D'ANCRAGE

3.1 Le management stratégique

3.1.1 Définition

Le système de management stratégique est un système d'activités permettant aux gestionnaires de l'entreprise Imagem de concentrer toutes les activités actuelles de planification, d'allocation des ressources, de production, de vente et promotion, de développement de nouveaux produits et de contrôle, vers un même but: celui d'assurer le réalignement stratégique et les réajustements de mise en oeuvre stratégique, par des actions concertées. Le tout devra respecter l'intérêt des propriétaires, l'expérience de l'entreprise, la rentabilité des produits et le marché tel qu'il se répartit entre les divers agents économiques.

\section{1 .2 Ancrage}

a) Propriétaires du système Image $m$

b) Environnement:

Le marché de la publicité et de la production audiovisuelle pour la région de la Capitale nationale. 
c) Clients:

Les trois actionnaires majoritaires et gestionnaires de l'entreprise Imagem, soit Daniel Brazeau, Marc Gendron et Jean-Guy Proulx.

d) Transformation:

Permet de concentrer les activités actuelles de planification; d'allocation de $s$ ressources; de vente et promotion; de développement de nouveaux produits et de contrôle, toutes vers un même but: celui d'assurer le réalignement stratégique et les réajustements de mise en oeuvre stratégique, par des actions concertées.

e) Ac teurs:

Les trois actionnaires majoritaires de l'entreprise;

La Caisse populaire de Hull;

Le personnel et certains pigistes de 1 'entreprise;

Les autres actionnaires minoritaires ou membres du Conseil d'administration.

f) Point de vue:

Pour assurer la survie de l'entreprise à moyen terme, il s'avère nécessaire que tous les efforts de vente, de production, de 
contrôle et autres soient mieux orchestrés. On favorisera ainsi la rentabilité de 1 'entreprise. 


\begin{abstract}
Lorsquion passe en revue les principaux problèmes de l'entreprise Imagem, il devient évident que la solution à adopter doit toucher toutes les fonctions de l'entreprise; par une planification stratégique plus rigoureuse et par des actions cohérentes adaptées aux ressources de l'entreprise. On parle alors du réalignement stratégique et des réajustements de mise en oeuvre stratégique.
\end{abstract}

Il s'avère finalement intéressant, à la lumière d'une revue de littérature adéquate, de proposer l'élaboration d'un système de management stratégique pour atteindre les objectifs fondamentaux énoncés ci-haut. 
142

I X

LA CONCEPTUALISATION ET LA COMPARAISON DU SYSTEME PERTINENT 
Le présent chapitre cède la place aux étapes de conceptualisation et de comparaison de la méthode.

Dans un premier temps, nous décrirons les activités de base d'un système de management stratégique dans notre modè le conceptuel. Bien que les écrits de messieurs chicha et Bamberger constituent la source de notre apprentissage des systèmes de management stratégique, la construction de notre modèle se base plus particulièrement sur ceux de Bamberger car celui-ci présente de façon plus simplifiée, selon nous, les activités nécessaires d'un processus de management stratégique complet. Il faut retenir qu'à cette étape de notre intervention, le modèle s'adresse avant tout aux décideurs et aux utilisateurs du système qui doivent en comprendre les particularités et les finalités. Nous procéderons ensuite à la validation du système retenu. Puis, nous poursuivrons notre démarche par la comparaison entre le modèle conceptuel soumis et le savoir-faire de l'entreprise à ce niveau. Finalement, nous porterons nos efforts sur les changements potentiels issus de la comparaison. 


\section{SYSTÈME DE MANAGEMENT STRATÉGIQUE}

Un système de management stratégique permet à l'entreprise qui le met en oeuvre, d'orienter ses activités vers le futur. Voici comment Ingolf Bamberger le définit: "Son idée de base est que l'entreprise devrait continuellement scruter les développements actuels et futurs dans son environnement et développer un concept stratégique qui guide et coordonne son évolution: le management stratégique est le guidage de l'évolution à long terme de l'entreprise par un concept stratégique". (1)

Le management stratégique repose sur deux principes: d'abord la contingence, i.e. le fait qu'il n'existe pas une bonne structure, une bonne stratégie, un bon mode de gouvernement, tout est affaire de situation spécifique ensuite, la cohérence entre le style de gouvernement, la - stratégie, la structure et la culture.

Le managent stratégique se base sur l'idée qu'une entreprise ne peut pas uniquement s'adapter à l'environnement par un choix adéquat de produits et de marchés, mais aussi par la gestion harmonieuse de ses ressources internes. 
On dit même que toutes les petites entreprises doivent et peuvent le réaliser. Leur faible mobilité stratégique constitue une raison plus que valable d'investir des énergies dans un processus de management stratégique, car les réorientations stratégiques fondamentales nécessitent la transformation et le développement des capacités internes pour parer aux changements futurs du marché.

Le management stratégique se fait donc le promoteur de l'apprentissage managérial. En effet, il permet de développer chez les propriétaires d'entreprises des comportements de réflexion sur l'avenir, d'amélioration de la connaissance de l'environnement et de ses facteurs critiques, d'analyse systématique des situations stratégiques, etc.

Le système de management stratégique de l'entreprise Imagem pourrait donc se composer des quatre sous-systèmes suivants: l'analyse stratégique, le développement du concept stratégique, le processus de mise en oeuvre stratégique et le contrôle stratégique.

(voir la figure 9.1) 


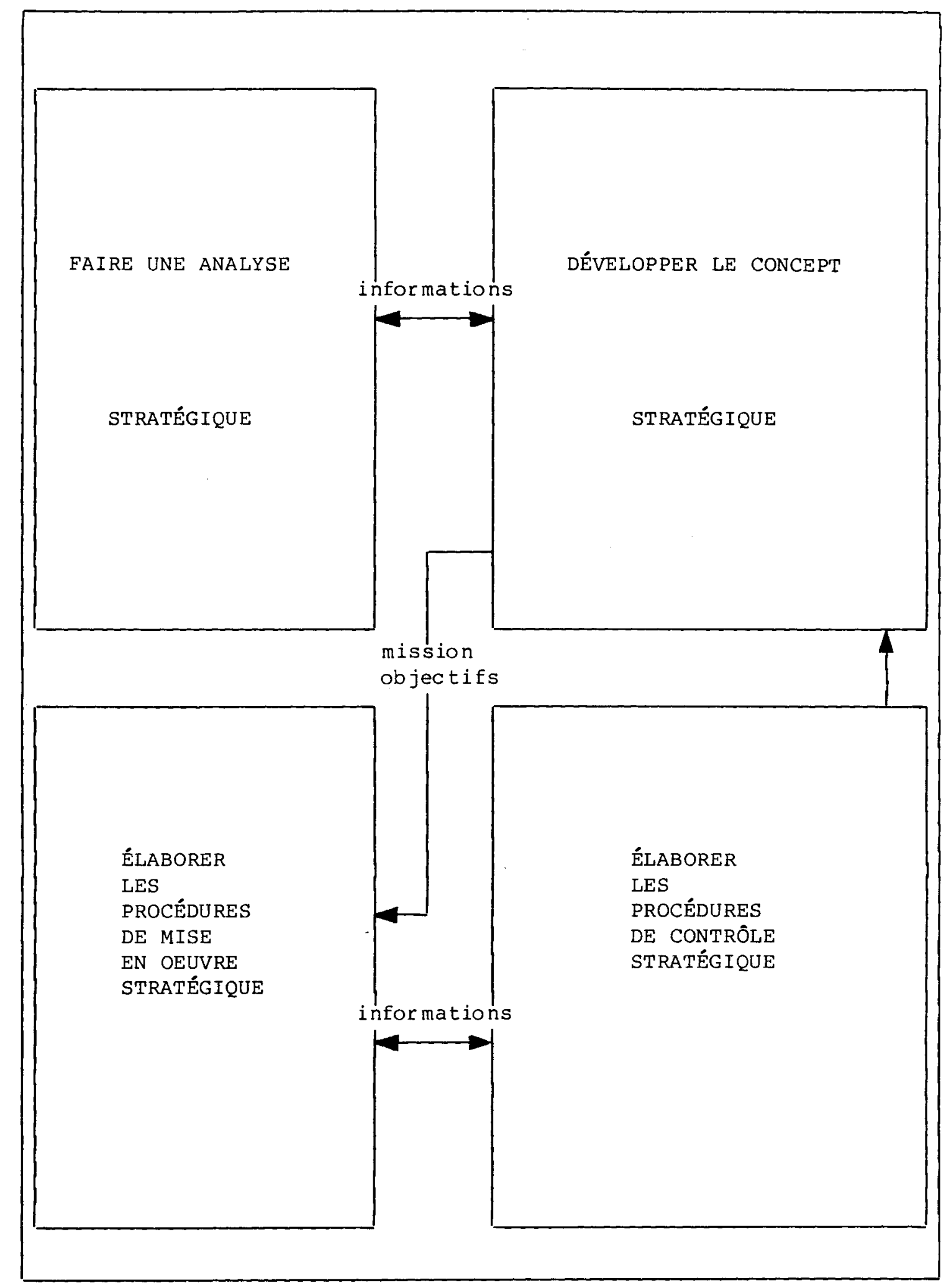

Figure 9.1 Système de management stratégique 


\subsection{Analyse stratégique}

L'analyse stratégique constitue la phase préliminaire de l'ensemble du processus de management stratégique, puisqu'elle permet de susciter la recherche d'informations pertinentes avant de procéder au développement du concept stratégique. Selon Bamberger: "I'analyse stratégique est un processus permanent de recherche et d'étude de l'information"(1). Ces informations se rapportent aux opportunités et menaces de l'environnement, à la position de notre entreprise dans le secteur, aux particularités du marché visé ou aux tendances dans la demande.

En ce qui concerne l'environnement, l'analyse ne doit pas se limiter aux marchés actuellement servis par l'entreprise. Elle doit aussi englober les conditions environnementales à un niveau plus large, qui pourraient influencer l'évolution des marchés ou créer de nouvelles opportunités.

Cette analyse pourrait s'attarder plus spécifiquement aux changements sociaux ou comportement des consommateurs et leur impact sur la demande du produit; aux facteurs clés de succès sur les marchés; aux forces et faiblesses de l'entreprise pour chacun de ses produits; etc. 
Par exemple, les changements affectant la segmentation du marché, la sensibilité de la clientèle aux prix, l'intérêt face au service après vente, etc., peuvent constituer des menaces ou opportunités, faire disparaître ou créer des segments de marché spécifiques, ou modifier les facteurs clés de succès. Pour sa part, l'étude des facteurs clés de succès actuels et leurs changements potentiels peut aider la petite entreprise, à développer des compétences distinctives pour s'accaparer ou conserver une niche de marché.

A la figure 9.2, on peut distinguer quatre activités qui se rattachent à l'analyse stratégique: l'analyse de l'environnement, l'évaluation de la position de l'entreprise, l'analyse des marchés visés et l'évaluation de la tendance de la demande.

\subsection{Déve loppement du concept stratégique}

Selon Bamberger "le développement du concept stratégique inclut la génération, l'analyse, l'évaluation et la sélection des options stratégiques. C'est un processus créatif ainsi que l'objet d'analyses, de procédures et de méthodes systématiques."(1) La créativité permet aux décideurs de sortir quelque peu des sentiers battus dans leur choix de stratégies alors que les analyses systématiques permettent d'étudier leurs conséquences et leur niveau de faisabilité. 


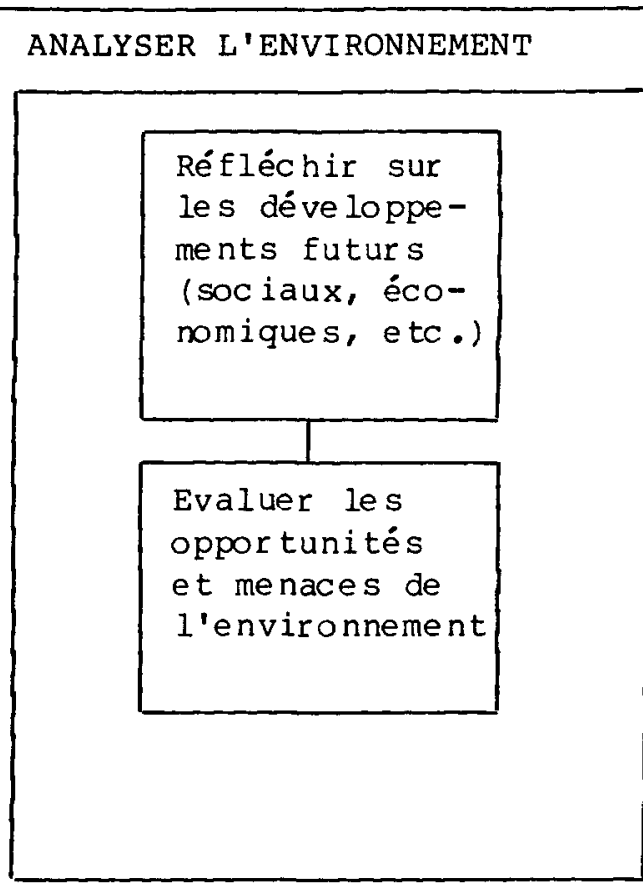

ANALYSER LES MARCHÉS VISÉS

Analyser les facteurs clés de succès dans ce marc hé

Décrire la conc urre nce
ÉVALUER LA POSITION DE L'ENTREPRISE

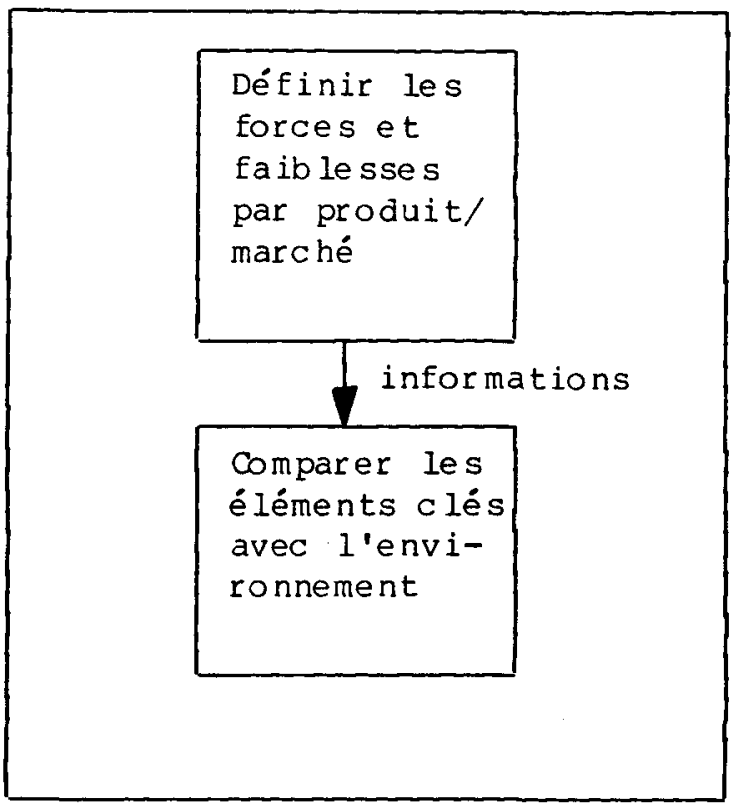

ÉVALUER LES TENDANCES DE LA DEMANDE

Evaluer l'intérêt de s consommateur $\mathrm{s}$ et leurs comportements d'achat

Evaluer I'élasticité de la demande 
Le développement du concept stratégique consiste en deux processus fondamentaux: d'abord la définition du cadre stratégique avec la mission, les principes généraux, les objectifs gé néraux et la posture stratégique, ensuite le plan stratégique pour chaque produit/marché visé par l'entreprise. Dans les petites entreprises, les valeurs personnelles des propriétaires, leurs attitudes et leurs intérêts, se reflètent directement sur la formulation des objectifs. Par ailleurs, dans l'élaboration du plan stratégique, on doit choisir les produits/marchés qui tiennent compte à la fois de l'environnement, de la position concurentielle de l'entreprise et du cadre stratégique précédemment adopté. Pour chacun des produits/marchés retenus, on devra inévitablement se questionner sur son importance relative dans l'ensemble des activités de notre firme, des ressources nécessaires et des compétences distinctives à développer. Bien que les petites entreprises disposent en principe d'un large éventail de stratégies potentielles, on doit échelonner les changements fondamentaux sur une période de temps suffisante pour permettre le changement et le développement des capacités inter nes.

La figure 9.3 explique de façon détaillée, l'ensemble des activités se rattachant au développement du concept stratégique. 


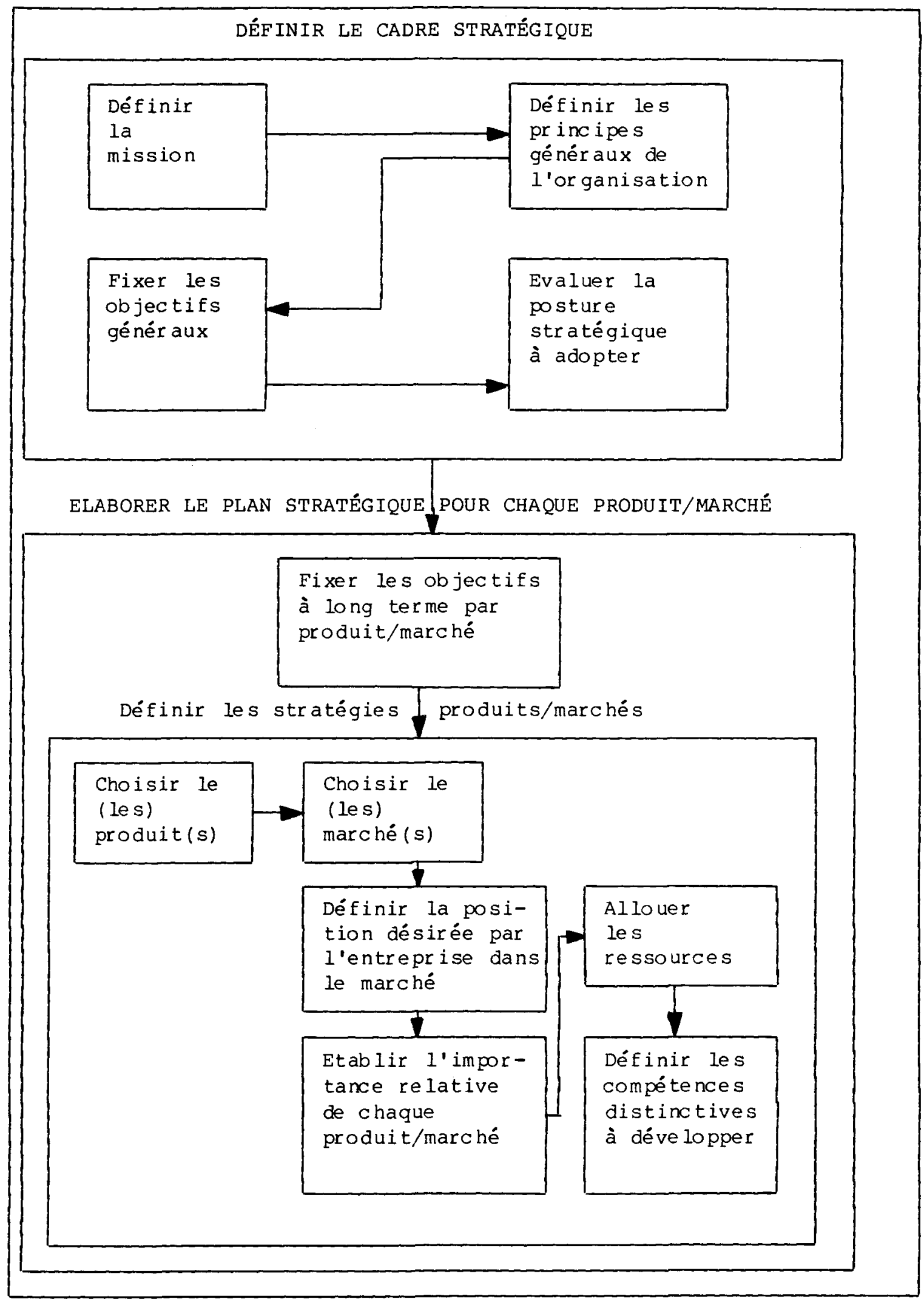

Figure 9.3 Sous-système de développement du concept stratégique 


\subsection{Mise en oeuvre stratégique}

Le processus de mise en oeuvre stratégique permet la fixation de stratégies et de sous-stratégies fonctionnelles, l'élaboration de plans opérationnels, de programmes, de budgets prévisionnels qui mènent ensuite à des actions concrètes. On peut aussi y analyser la nécessité de modifier la structure organisationnelle et adapter le système d'information et de contrôle aux besoins de l'organisation.

Les stratégies fonctionnelles relèvent des disciplines qu'elles concernent soit entre autres en: finance, marketing, personnel et production. Elles s'apparentent davantage à des activités d'exécution qu'à la stratégie de l'entreprise, sans être qualifiables d'activités quotidiennes et répétitives. Pour leur part, les sous-stratégies fonctionnelles se composent de chaque partie des changements décidés dans le cadre de la stratégie globale. On regroupe les sous-stratégies fonctionnelles selon les fonctions dont elles relèvent.

Le champ de la mise en oeuvre stratégique ne se limite donc pas aux frontières de la stratégie générale. Il s'étend aux stratégies et aux sous-stratégies fonctionnelles qui ne requièrent pas l'intervention de la direction générale, mais plutôt celle des divers niveaux de dirigeants ou de cadres. 
Précisons finalement qu'au stade de mise en oeuvre stratégique, les acteurs se questionnent sur la cohérence entre les nombreux plans opérationnels et le concept stratégique, de même que sur les conséquences financières des actions à entreprendre.

La figure 9.4 fournit des détails sur le processus de mise en oeuvre stratégique.

\subsection{Contrôle stratégique}

Le contrôle stratégique se compose du contrôle de la mise en oeuvre stratégique et de l'étude comparative entre les résultats et les objectifs fixés; aussi bien dans le développement du concept stratégique que dans le processus de mise en oeuvre stratégique. Premièrement, I'élaboration d'outils de contrôle des activités de mise en oeuve stratégique permet de réévaluer et réajuster au besoin le processus de mise en oeuvre stratégique. Deuxièmement, les états financiers et rapports spéciaux périodiques permettent de comparer les résultats aux objectifs visés. Ensuite, l'analyse des écarts permet d'évaluer la situation globale de I'entreprise. 


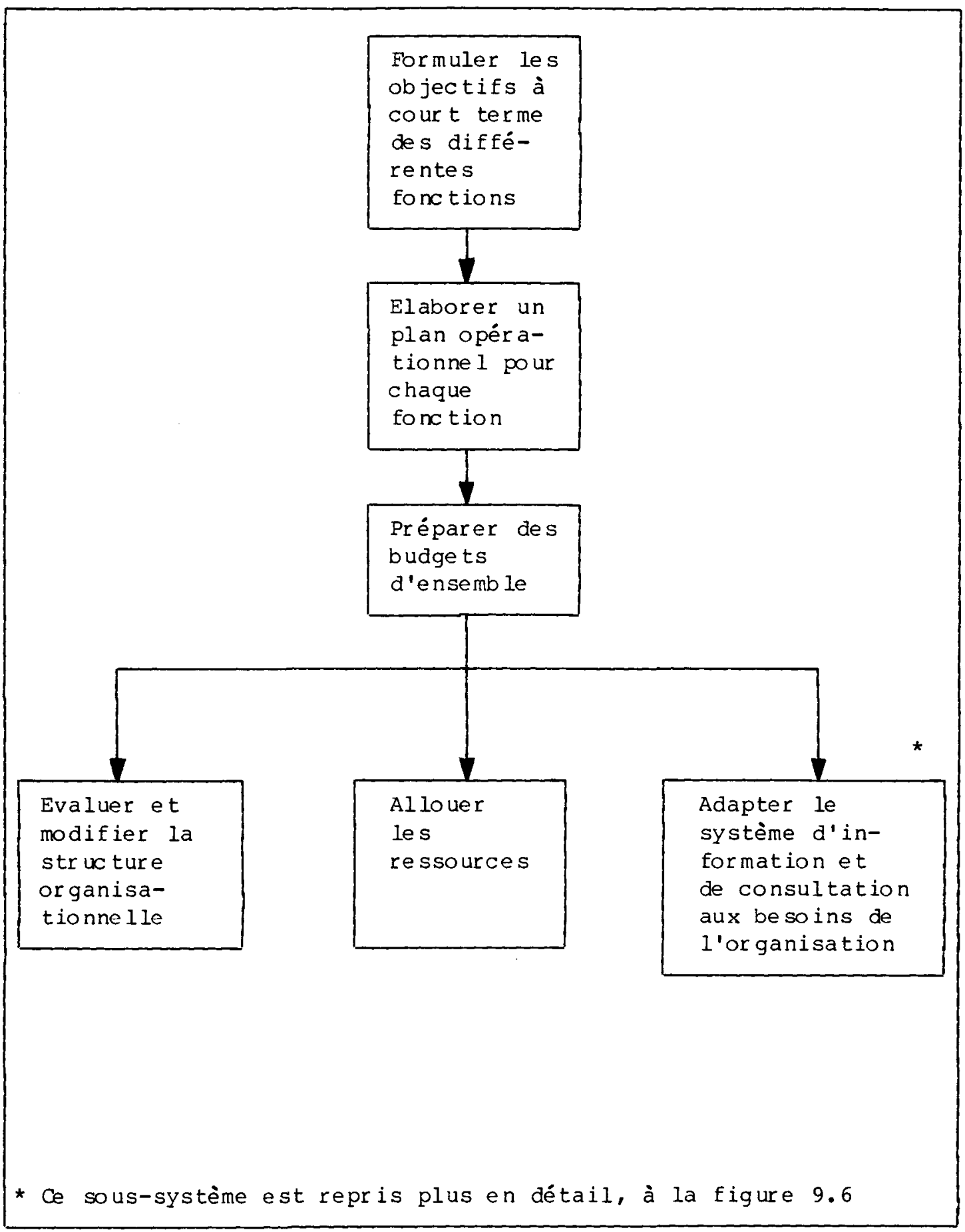

Figure 9.4 Sous-système de mise en oeuvre stratégique 
L'ensemble du système de contrôle stratégique entre alors en interaction avec le système de développement du concept stratégique et celui de mise en oeuvre stratégique.

(voir figure 9.5)

2. SOUS-SYSTEME D'INFORMATION ET DE CONSULTATION

Ce système se compose des quatre étapes successives suivantes: la détermination des forces et faiblesses du système actuel; la définition des besoins de l'organisation; Ia formulation d'objectifs et finalement l'élaboration d'un plan opérationnel d'information et de consultation pour chaque système d'activités qui en a besoin.

(voir la figure 9.6) 


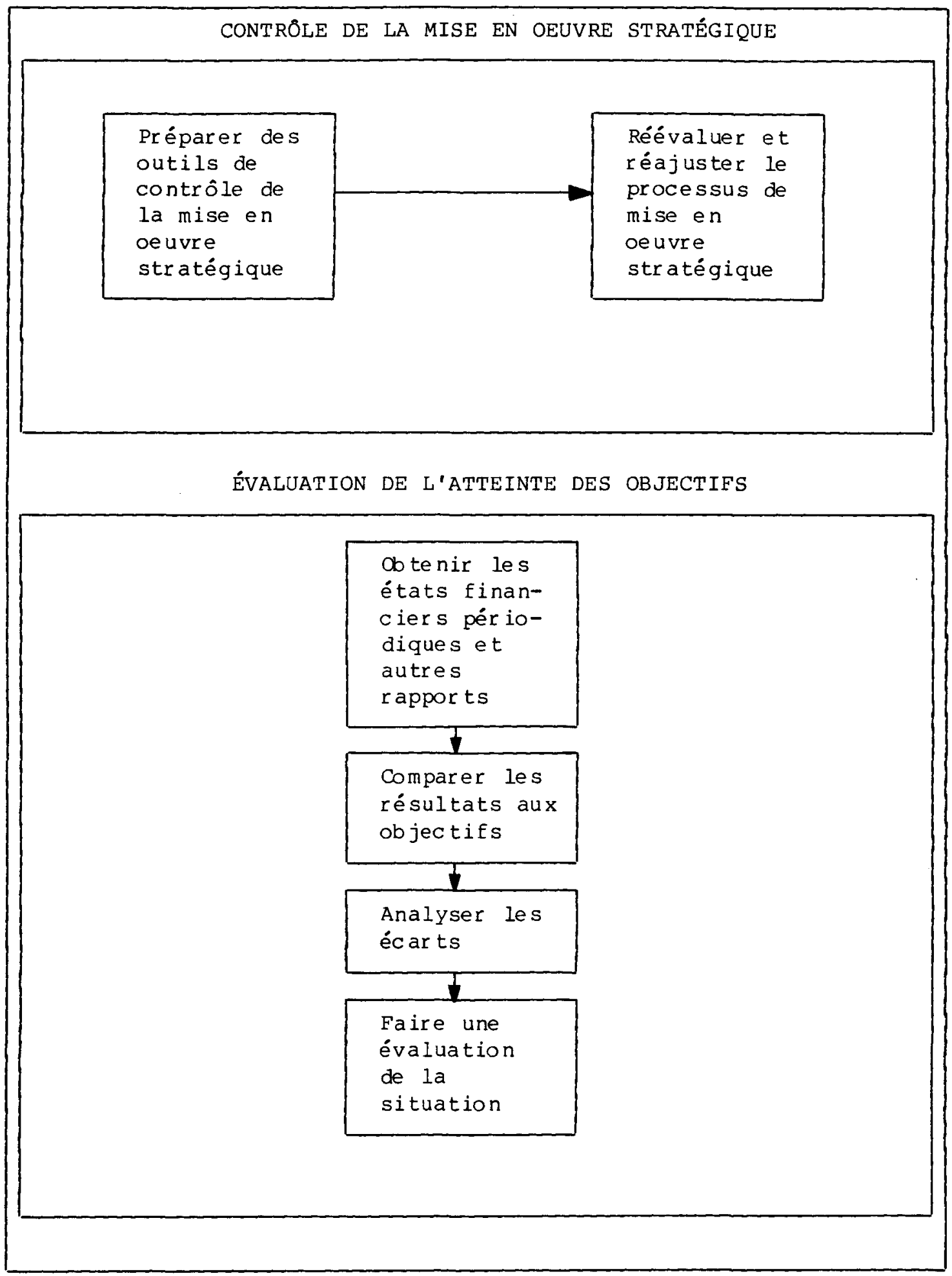

Figure 9.5

Sous-système de contrôle stratégique 


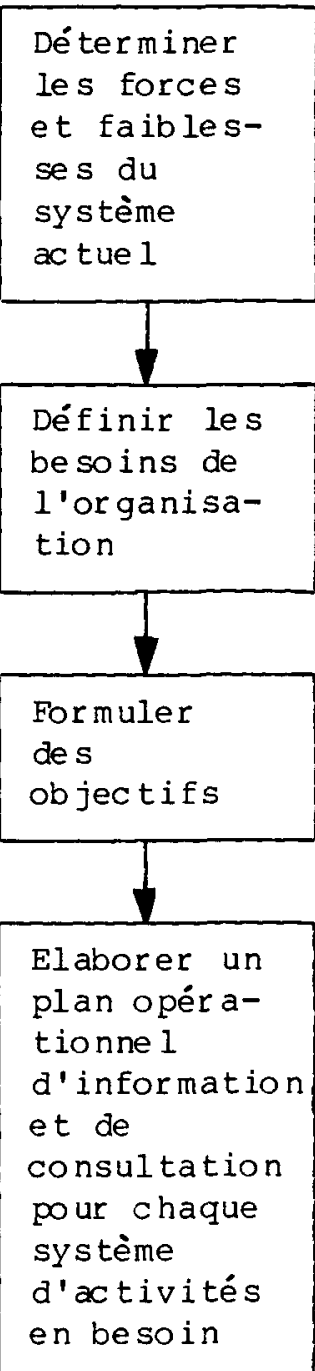

Figure 9.6 Sous-système d'information et de communication 


\section{VALIDATION DU MODELE CONCEPTUEL}

P.B. Checkland propose huit caractéristiques essentielles que devrait contenir un système d'activités humaines. En ce qui concerne le système de management stratégique énoncé précédemment, cela se traduit de la façon suivante:

Carac téristiques

essentielles à

tout système

1. avoir un objectif

2. avoir des mesures de performances

3. avoir des sous-systèmes
Transposition à notre système de management stratégique

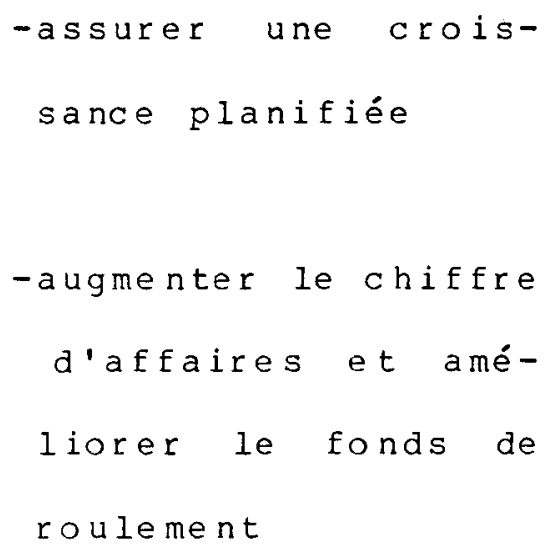

-établir des soussystèmes d'analyse stratégique, de dé ve loppement du concept stratégique, de mise en oeuvre stratégique et de contrôle 
Caractéristiques

essentielles à

tout système

4. avoir des interrelations

5. être un système ouvert, rattaché à l'environnement
Transposition à notre système de management stratégique

- améliorer le processus d'information et de prise de déci sions :

-tenir compte de l'environnement concurrentiel et du marché, dans l'établissement des soussystèmes de développement et d'opérationnalisation

\footnotetext{
- augmenter les ressources financières et améliorer la banque de ressources humaines
} 
7. avoir des preneurs de décisions

8. présenter une certaine garantie de continuité
- améliorer le processus de prise de décision des trois propriétaires

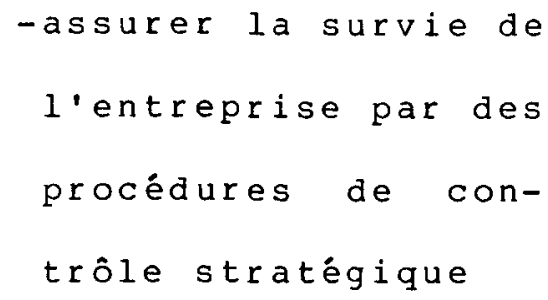

4. COMPARAISON ENTRE LE MODÈLE CONCEPTUEL ET LE SAVOIR-FAIRE DE L'ENTREPRISE $\grave{A}$ CE NIVEAU

Afin de faciliter l'analyse comparative, nous reprenons ici les quatre activités de base du modèle conceptuel que nous pourrons plus facilement comparer avec le savoir-faire de l'entreprise.

\subsection{Analyse stratégique}

A ce niveau, nous ne dénotons pas de manque sérieux chez Imagem. Les gestionnaires connaissent relativement bien le marché et la concurrence. La seule action correctrice qui peut alors être proposée, se rattache à l'analyse et au consensus à obtenir sur les forces et faiblesses de l'entreprise. 


\subsection{Dé ve loppe ment du concept stratégique}

La définition du cadre stratégique ne pose aucun problème aux propriétaires de l'entreprise. Par contre, au niveau de l'élaboration du plan stratégique, nous pouvons prévoir certaines difficultés dans l'allocation des ressources.

\subsection{Mise en oeuvre stratégique}

Nous constatons que les activités de mise en oeuvre stratégique font défaut chez Imagem. L'entreprise s'est souvent limitée à la formulation d'objectifs sans élaborer de plans opérationnels détaillés, de budgets prévisionnels et sans planifier de façon rigoureuse l'impact des choix sur l'allocation des ressources, la structure organisationnelle et Le système d'information.

\subsection{Contrôle stratégique}

Cette dernière activité représente une étape importante pour permettre à l'ensemble du processus de management stratégique de se renouveler. De plus, il nous apparait évident que d'une part, l'entreprise n'est pas tellement outiliée pour effectuer le contrôle de la mise en oeuvre stratégique et que d'autre part, elle requiert le soutien de personnel qualifié en comptabilité pour la compilation et l'analyse des données financières. 


\section{ACTIONS CORRECTRICES A ENTREPRENDRE}

Considérant la somme de travail que représente un processus complet de management stratégique, nous avons jugé bon de concentrer les correctifs à entreprendre en majeure partie sur l'analyse stratégique, la mise en oeuvre stratégique et le contrôle stratégique. Ainsi, nous pouvons énumérer les correctifs suivants: amélioration de l'analyse stratégique par une description des forces et faiblesses de l'entreprise pour chaque produit offert, réalignement de la stratégie, élaboration d'un plan stratégique détaillé et élaboration d'outils de contrôle de la mise en oeuvre stratégique. 
N'oublions pas que le modèle conceptuel énoncé dans la première partie de ce chapitre sert d'abord, à identifier les activités nécessaires à l'existence d'un système de management stratégique; puis comme point de comparaison avec le savoirfaire de l'entreprise afin de proposer des actions correctrices valables.

Dans le cas d'Imagem, ces actions correctrices devront ainsi se concentrer sur une meilleure connaissance de l'entreprise par ses dirigeants, sur le réalignement de la stratégie, sur l'élaboration de plans stratégiques détaillés et finalement, sur l'élaboration d'outils de contrôle de la mise en oeuvre stratégique. 
Ce chapitre se propose de faire passer les intervenants de la théorie à la pratique.

Dans un premier temps, nous proposons aux personnes
impliquées dans le processus de management stratégique, des
outils de travail qui faciliteront leurs démarches. Ces outils
s'appuient sur le modèle conceptuel qui continue de servir de
cadre de discussion.

Ensuite, nous é noncerons d'autres recommandations qui pourront faire partie intégrante du plan opérationnel. Finalement, nous exposerons le plan de management stratégique adopté par les propriétaires de l'entreprise. 


\section{OUTILS DE TRAVAIL}

Trois modèles d'analyse ou de contrôle ont été soumis aux gestionnaires: un tableau comparatif des forces et faiblesses de l'entreprise par produit, un tableau synthèse du plan opérationnel et un calendrier de la mise en oeuvre stratégique. on les retrouve aux tableaux $10.1,10.2$ et 10.3 . 
167

Tableau 10.1 Forces et faiblesses de l'entreprise

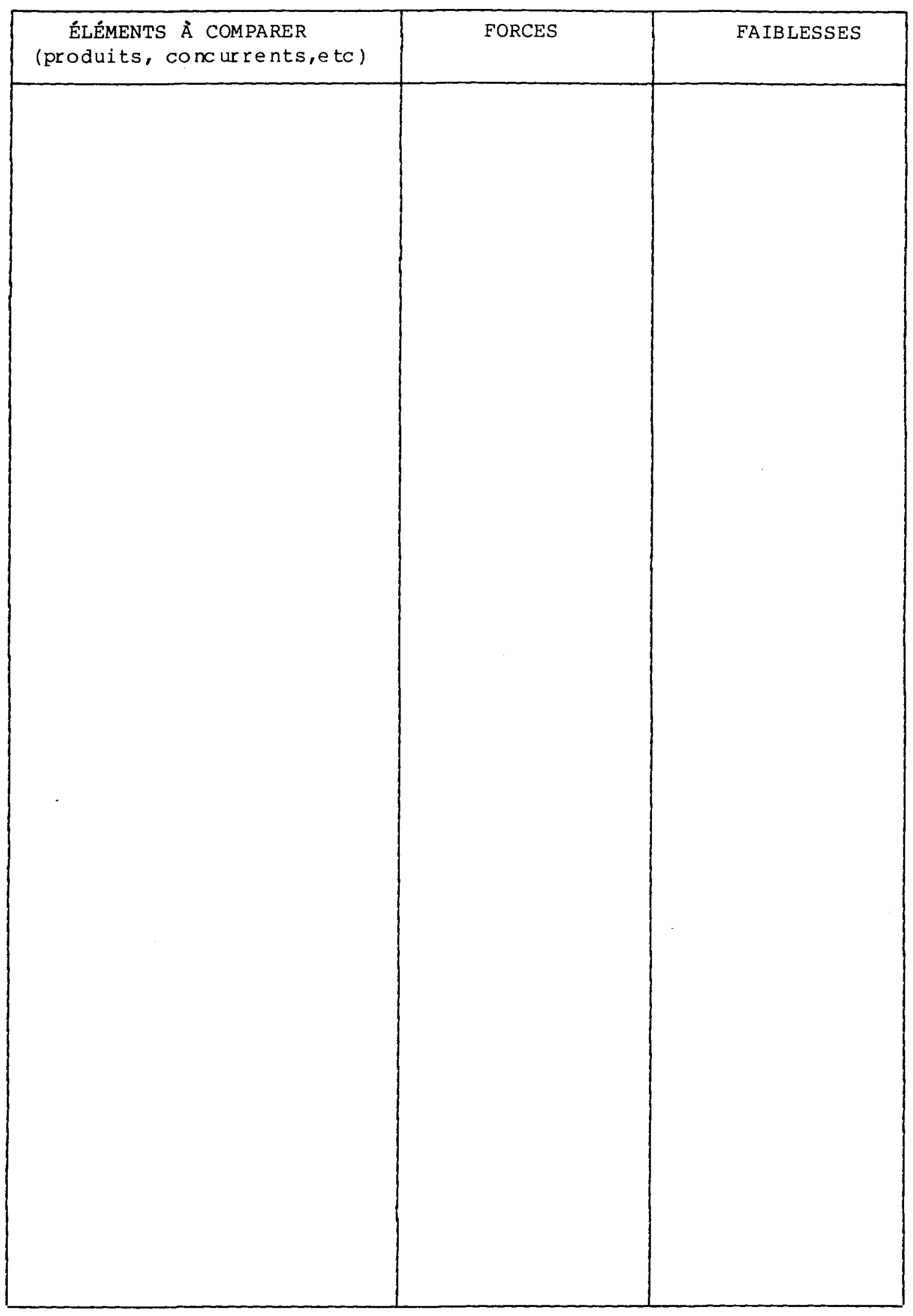


Tableau 10.2 Synthèse du plan opérationne 1

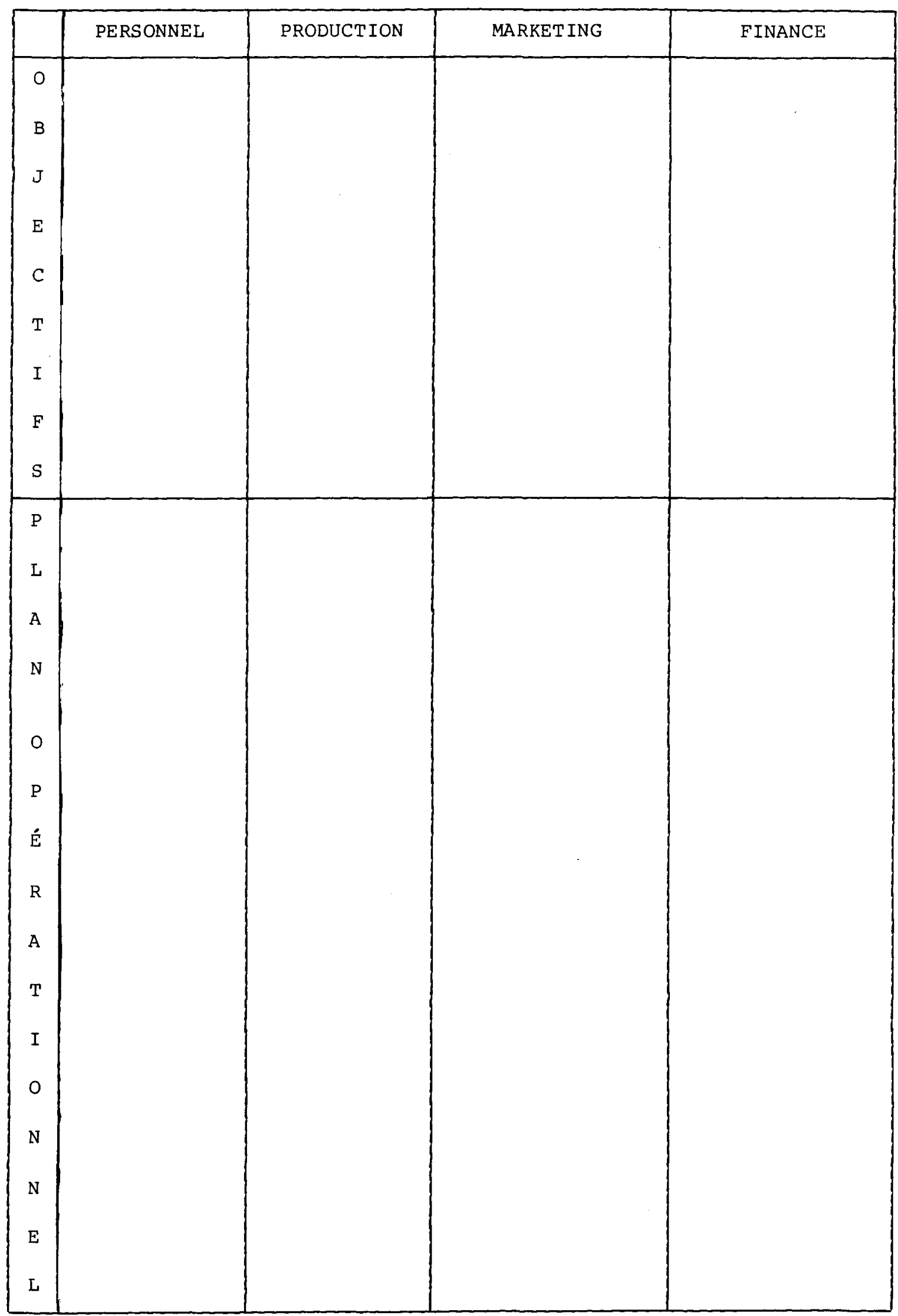


Tableau 10.3 Calendrier de mise en oeuvre stratégique

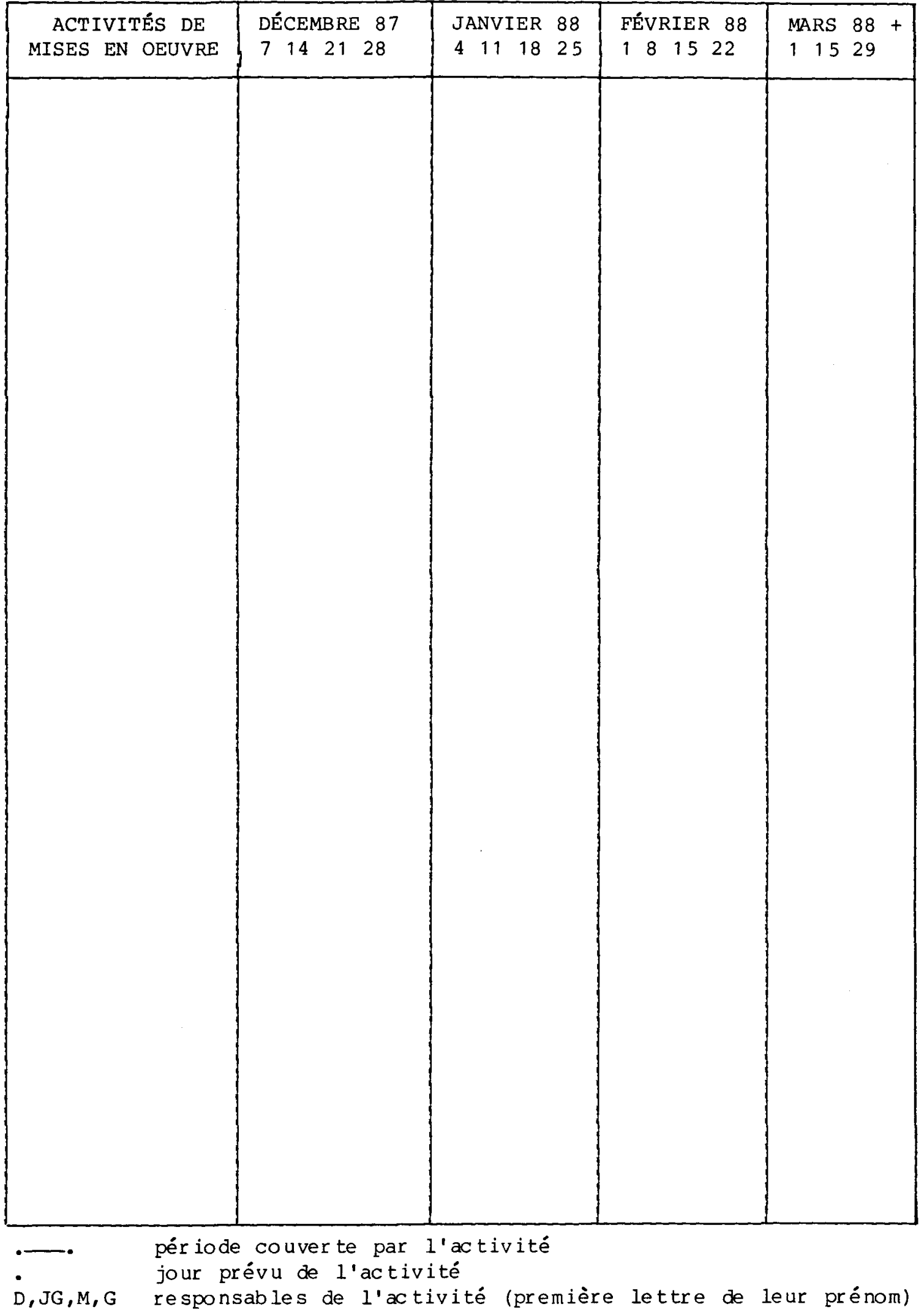




\section{AUTRES RECOMMANDATIONS}

Une première catégorie de recommandations concerne la planification et la réorganisation financière, à savoir la vente d'équipements sous-utilisés et inutiles, la finalisation du dossier de l'assurance-feu, la préparation d'une rencontre avec le banquier et l'accélération de la facturation et de la perception des comptes à recevoir.

Une deuxième catégorie de recommandations touche la gestion des ressources humaines. Il devient prépondérant de s'adjoindre du personnel compétent en conception et production audiovisuelle, en comptabilité, en gestion et en analyse de marchés et de faisabilité. Ces ressources peuvent être utilisées de façon ponctuelle ou permanente, dépendamment des capacités financières et des besoins de l'entreprise.

3. OPÉRATIONNALISATION DU SYSTÈME DE MANAGEMENT STRATÉGIQUE

\subsection{Analyse stratégique}

Au cours du diagnostic de l'entreprise, nous avons procédé à une analyse de l'environnement, des marchés et des tendances de la demande. Vous trouverez ces informations au chapitre III. Nous nous contenterons d'évaluer la position concurrentielle d'Imagem dans la présente partie. Pour ce 


\begin{abstract}
faire, nous décrirons les forces et faiblesses de l'entreprise pour chacun des produits qu'elle a offerts au cours de la dernière année. Cette évaluation sera ausi réalisée en tenant compte des facteurs clés de succès pour chaque produit.

Le tableau 10.4 reproduit les résultats de cette analyse.
\end{abstract}


Tableau 10.4
Forces et faiblesses d'Imagem

IMAGEM

FA IB LESSES

- connaissances des produits de la concurrence

-manque $d$ 'expérience

- manque de contacts avec

le milieu

-manque de connaissance du marché

-difficulté de bien raconter l'histoire

-produit de qualité moyenne - inexpérimentation de l'équipe -méconnaissance des techniques de production (effets optiques)

.intérêts des gestionnaires pour ces produits

-force de vente -prix bas
- peu d'intérêt de la part des gestionnaires -manque de compétence et d'expertise -produit de qualité moyenne 


Tableau $10.4 \quad$ Forces et faiblesses d'Imagem (suite)
IMAGEM

FORCES

FAIBLESSES

TRAVAUX

D'IMPRIMERIE

SERVICES

TECHNIQUES

ET LOCATION

\begin{tabular}{|c|c|}
\hline & $\begin{array}{l}\text {-peu d'intérêt de la } \\
\text { part des gestionnaires } \\
\text {-manque de compétence } \\
\text { et d'expertise } \\
\text {.équipe de production } \\
\text { plus ou moins rodée } \\
\text {-méconnaissance du } \\
\text { processus de pro- } \\
\text { duction } \\
\text {-hors du champ de } \\
\text { compétence des } \\
\text { gestionnaires }\end{array}$ \\
\hline $\begin{array}{l}\text {-pigistes } \\
\text {.prix compétitifs }\end{array}$ & $\begin{array}{l}\text { - peu d'intérêt de la } \\
\text { part des gestionnaires }\end{array}$ \\
\hline $\begin{array}{l}\text { éventail d'équi- } \\
\text { pements } \\
\text {.prix compétitifs }\end{array}$ & -marché mince \\
\hline
\end{tabular}




\subsection{Dé ve loppe ment du concept stratégique}

$$
\text { 3.2.1 Le cadre stratégique }
$$

Les gestionnaires d'Imagem s'accordent pour donner à I'entreprise une mission de conception, production et services de consultation dans le secteur de l'audiovisuel.

Le marché primaire se concentre dans la région de la Capitale nationale, alors que le marché secondaire s'étend à 1 'ensemble du Québec.

Les principes généraux qui encadrent l'ensemble du concept stratégique se définissent comme suit:

$$
\begin{aligned}
& \text { 1. fournir aux gestionnaires et actionnaires } \\
& \text { majoritaires de l'entreprise des conditions de } \\
& \text { travail adéquates (salaires, avantages sociaux, défis } \\
& \text { à relever, etc.). } \\
& \text { 2. renforcer l'identification de l'entreprise dans le } \\
& \text { marché de l'audiovisuel. Imagem est la seule entre- } \\
& \text { prise qui offre des services complets de conception, } \\
& \text { production et consultation en audiovisuel, dans } \\
& \text { l'outaouais québécois. }
\end{aligned}
$$


Les objectifs généraux se définissent comme suit:

-orienter la production audiovisuelle vers les produits de moye nne gamme

-concentrer la gamme de produits et services en fonction des compétences distinctives de l'entreprise

-offrir des produits qui rencontrent les trois critères suivants: professionnalisme, originalité et satisfaction du client

- atteindre un chiffre d'affaires d'environ $235000 \$$ et 245 $000 \$$ respectivement pour 1 'an 1 et pour 1 'an 2

Finalement, en ce qui concerne la posture stratégique, l'entreprise entend adopter un style un peu plus agressif que dans le passé afin d'atteindre le chiffre d'affaires espéré.

\subsubsection{Le plan stratégique}

La gamme de produits qu'Imagem compte offrir à ses clients se limitera aux vidéos industriels ou "broadcast", aux diaporamas et au dé veloppement de nouveaux produits. Elle offrira bien sûr, pour ces produits, des services professionnels de consultation et de production en communication audiovisue Ile. 
Le marché auquel l'entreprise s'adresse, regroupe les entreprises privées, les organismes sans but lucratif, les organismes publics et para-publics, les agences de publicité et les télédiffuseurs. Leur caractéristique commune est le souci d'obtenir un produit de communication audiovisuelle de moyenne gamme.

L'importance relative de chacun des produits offerts, en ce qui a trait à leur contribution au chiffre d'affaires, se répartit comme suit: les vidéos, $50 \%$; les diaporamas, 20\%; et le développement de nouveaux produits $30 \%$.

Les compétences distinctives à développer pour les vidéos consistent à canaliser des ressources humaines compétentes dans l'entreprise. Pour les diaporamas, il s'agit de développer des ressources pour la réalisation de productions d'envergure. Finalement en ce qui concerne le développement de nouveaux produits, on doit faire preuve d'originalité et surtout, répondre à des besoins actuellement non comblés chez les clients potentiels. 


\subsection{Mise en oeuvre stratégique}

La mise en oeuvre stratégique représente une activité où les grandes orientations se traduisent en actions. Chez Imagem, ces actions s'appuient d'abord sur un plan opérationnel présenté au tableau 10.5. Puis l'élaboration de budgets prévisionnels permet de réajuster le plan pour tenir compte des capacités financières de l'entreprise. Finalement, une allocation adéquate des ressources, appuyée d'une restructuration organisationnelle, vient compléter le tout. 
Tableau 10.5 Objectifs et plan opérationnel d'Imagem

\begin{tabular}{|c|c|c|}
\hline \multirow[b]{2}{*}{ OBJECTIFS } & PERSONNEL & PRODUCT ION \\
\hline & $\begin{array}{l}\text {-augmenter la qualité et la } \\
\text { quantité des ressources humaines } \\
\text { disponibles pour la conception et } \\
\text { la production audiovisuelle. } \\
\text {.s'adjoindre du personnel com- } \\
\text { pétent à la comptabilité et au } \\
\text { secrétariat. } \\
\text {-améliorer les conditions de } \\
\text { travail de tout le personnel } \\
\text { (gestionnaires et autres). }\end{array}$ & $\begin{array}{l}\text {-améliorer la qualité des } \\
\text { produits. } \\
\text { - améliorer les méthodes et } \\
\text { les processus de production }\end{array}$ \\
\hline $\begin{array}{c}\text { PLAN } \\
\text { OPÉRATIONNEL }\end{array}$ & $\begin{array}{l}\text {-augmenter les salaires des } \\
\text { gestionnaires. } \\
\text {.embaucher un comptable. } \\
\text {.élaborer un contrat d'embauc he } \\
\text { type pour les pigistes. } \\
\text {.embaucher un consultant externe } \\
\text { pour développer le projet } \\
\text { d'auto-construction. } \\
\text {-préparer une échelle salariale et } \\
\text { définir les conditions d'emploi } \\
\text { du personnel régulier et des } \\
\text { pigistes. } \\
\text { - redéfinir la structure } \\
\text { organisationnelle } \\
\text {.contacter les gens du milieu de } \\
\text { l'audiovisuel pour connaitre de } \\
\text { nouveaux pigistes disponibles. } \\
\text {.obtenir le portfolio de ces } \\
\text { pigistes et planifier une rencon- } \\
\text { tre avec eux. } \\
\text {-monter un dossier complet pour } \\
\text { chacun d'eux. }\end{array}$ & $\begin{array}{l}\text {-contacter les entreprises } \\
\text { qui offrent des services } \\
\text { spécialisés en production } \\
\text { audiovisuelle. } \\
\text { - former un comité de con- } \\
\text { trôle de la qualité et } \\
\text { l'intégrer au processus } \\
\text { de production. } \\
\text {-améliorer la gestion des } \\
\text { projets (réservations, } \\
\text { affectations des ressour- } \\
\text { ces, calendrier de pro- } \\
\text { duction, etc) }\end{array}$ \\
\hline
\end{tabular}




\begin{tabular}{|c|c|}
\hline MARKET ING & F INANCE \\
\hline $\begin{array}{l}\text {.accroitre le chiffre d'affaires } \\
\text { annuel de près de } 708 \text { pour } \\
\text { l'an } 1 \text { et de près de } 758 \text { pour } \\
\text { l'an2, comparativement aux } \\
\text { résultats du } 30 \text { juin } 1987 . \\
\text {. améliorer la visibilité de } \\
\text { l'entreprise. } \\
\text {. faire connaitre Imagem comme } \\
\text { un bureau de professionnels } \\
\text { de la communication audiovi- } \\
\text { suelle. }\end{array}$ & $\begin{array}{l}\text {-améliorer le fonds de roule- } \\
\text { ment de l'entreprise. } \\
\text {.consolider sa dette. } \\
\text {.améliorer la situation fi- } \\
\text { nancière globale. }\end{array}$ \\
\hline $\begin{array}{l}\text {. augmenter les efforts de re- } \\
\text { lations publiques. } \\
\text {. augmenter le budget des frais } \\
\text { de représentation. } \\
\text {. faire partie d'un plus grand } \\
\text { nombre d'associations locales } \\
\text { et régionales. } \\
\text {.étoffer le dossier de présen- } \\
\text { tation d'Imagem. } \\
\text {.concevoir un démonstrateur se- } \\
\text { lon les capacités de l'entre- } \\
\text { prise. } \\
\text { - solliciter la clientèle à } \\
\text { partir de concepteurs/produc- } \\
\text { teurs et non de vendeurs purs } \\
\text { et simples. } \\
\text {-s'adjoindre des partenaires } \\
\text { de taille dans le développement } \\
\text { de nouveaux produits. }\end{array}$ & $\begin{array}{l}\text {-préparer des états financiers } \\
\text { prévisionnels sur deux ans. } \\
\text {.vendre les équipements désuets } \\
\text {.finaliser le dossier de l'as- } \\
\text { surance-feu. } \\
\text {-s'informer des subventions } \\
\text { disponibles. } \\
\text {-rencontrer le banquier (re- } \\
\text { présentant de la Caisse } \\
\text { Populaire) pour lui soumettre } \\
\text { l'état de la situation et } \\
\text { consolider la dette à long } \\
\text { terme. } \\
\text {.intensifier la perception des } \\
\text { comptes à recevoir. } \\
\text {.rattraper le retard dans la } \\
\text { préparation des rapports de } \\
\text { Vidéotour. }\end{array}$ \\
\hline
\end{tabular}


3. 3. 1 Budgets prévisionnels

Les budgets prévisionnels permettent de constater que l'entreprise sortira de sa léthargie actuelle vers la fin du printemps 1988. Elle aura à ce moment remboursé les dettes à court terme, fournisseurs et impots, qui dataient de plusieurs mois et même parfois d'une année complète.

Vous trouverez aux annexes 7 à 10 , les états financiers prévisionnels pour la période de deux ans, soit du $1^{e r}$ novembre 1987 au 31 octobre 1989.

3.3.2 Allocation des ressources

Nous en résumons les éléments essentiels ci-dessous:

Les ressources humaines:

-Embauche d'un(e) comptable à temps complet;

- Constitution d'une banque de pigistes adéquate en ce qui concerne la conception et la production audiovisuelle;

- Embauche d'un(e) secrétaire de façon occasionnelle;

- Recours occasionnel à un consultant externe à l'entreprise pour divers conseils et analyse des états financiers. 
Les ressources financières:

- Vente d'équipements inutiles de près de $\$ 8500$

- Perception de l'assurance-feu d'une valeur de près de $\$ 13000 ;$

- Restructuration de la dette à long terme.

Les ressources matérielles et équipements:

- Aucun achat d'équipement à prévoir à ce stade;

- Utilisation de sous-contractants pour les productions de moyenne gamme, comme on l'a fait au cours des derniers mo is.

\subsection{3 structure organisationne 1 le}

Suite au départ d'un des trois associés, il devient nécessaire de revoir la structure organisationnelle dans son ensemble. Celle-ci doit permettre de soutenir les efforts élaborés dans les plans opérationnels.

Le plan d'action prévu à cet effet se définit comme suit:

- Redéfinir le rôle du conseil d'administration;

- Préparer un organigramme;

- Définir les responsabilités et tâches de chaque gestionnaire. 


\subsection{Contrôle stratégique}

Celui-ci implique d'abord le contrôle de la mise en oe uvre et ensuite, l'évaluation de l'atteinte des objectifs. Afin d'effectuer un contróle adéquat de la mise en oeuvre stratégique, nous avons d'abord préparé un calendrier d'activités que vous trouverez à 1 'annexe 11 .

Pour ce qui est de l'évaluation de l'atteinte des objectifs, on pourra procéder dès le mois de février 1988 . Les comparaisons entre les résultats réels et les objectifs de départ, appuyées par l'analyse des écarts, faciliteront l'évaluation de la situation à ce moment. On pourra au besoin, réajuster la mise en oeuvre stratégique et réévaluer l'ensemble du concept stratégique. 
L'opérationnalisation et l'implantation constituent les étapes finales du diagnostic organisationnel mais non les moindres. Pour le chercheur, on peut même parler de test de vérité. Il ne suffit pas de faire une bonne analyse de la situation et de proposer des solutions pour réussir, il faut que ces solutions soient acceptables pour une majorité des acteurs concernés et qu'elles soient réalisables.

Chez Imagem, nous avons d'abord concentré nos efforts dans l'élaboration d'outils simples permettant de faciliter le fonctionnement du système de management stratégique. Il s'est toutefois avéré inutile de pousser trop loincet exercice car un tel système fait beaucoup plus appel au processus décisionnel qu'à des processus d'action. Ensuite, quelques recommandations particulières relatives à la réorganisation financière et à la gestion des ressources humaines ont permis de donner le ton au plan d'action.

C'est ainsi que les propriétaires de l'entreprise ont refait le processus complet de management stratégique pour aboutir au plan d'action en question, lequel est réalisable sur une période de quatre mois. Nous devions toutefois respecter la mission de conception, production et services de consultation dans le secteur de l'audiovisuel que les dirigeants avaient adoptée. Nous devions aussi rencontrer les objectifs suivants: orienter la production audiovisuelle vers 
les produits de moyenne gamme, concentrer la gamme de produits et services en fonction des compétences distinctives de l'entreprise, offrir des produits professionnels, originaux et répondant aux attentes du client, et finalement, atteindre un chiffre d'affaires annue 1 de près de $\$ 240000$. 
185

PARTIE C 
LA CRITIQUE DE LA MÉThODOLOGIE 
Comme il a déjà été décrit dans la première partie du présent mémoire, la méthodologie des systèmes souples de Checkland forme la base de notre démarche de diagnosticintervention chez Imagem. "Cette méthode qui a été adaptée par Checkland à travers une trentaine d'expériences pour satisEaire la complexité des PMo (PME, Coopérative, Organisme communataire, Petite et Moyenne organisation publique et parapublique) fut conçue pour s'attaquer à des situations complexes et peu structurées. Sa démarche est avant tout systémique". (27) Ses différentes étapes seront abordées en détail dans le présent chapitre; à savoir ce que son auteur suggère pour faciliter le travail de tout chercheur et comment le chercheur (ici concerné) a vécu l'application d'une telle méthodologie. Une représentation graphique de cette méthode vous est transmise à la page suivante. Vous pourrez ainsi y retracer les principales étapes:l'élaboration du mandat; la description de l'image riche de l'entreprise; l'ancrage; la conceptualisation; la comparaison; l'opérationnalisation; l'implantation et le contrôle.

Finalement, nous profiterons de l'occasion pour aborder de façon très succincte les nombreux efforts de validation de la démarche qui lui permettent de se positionner tant bien que mal, dépendamment des auteurs, dans le monde des méthodes dites scientifiques. 
MANDAT

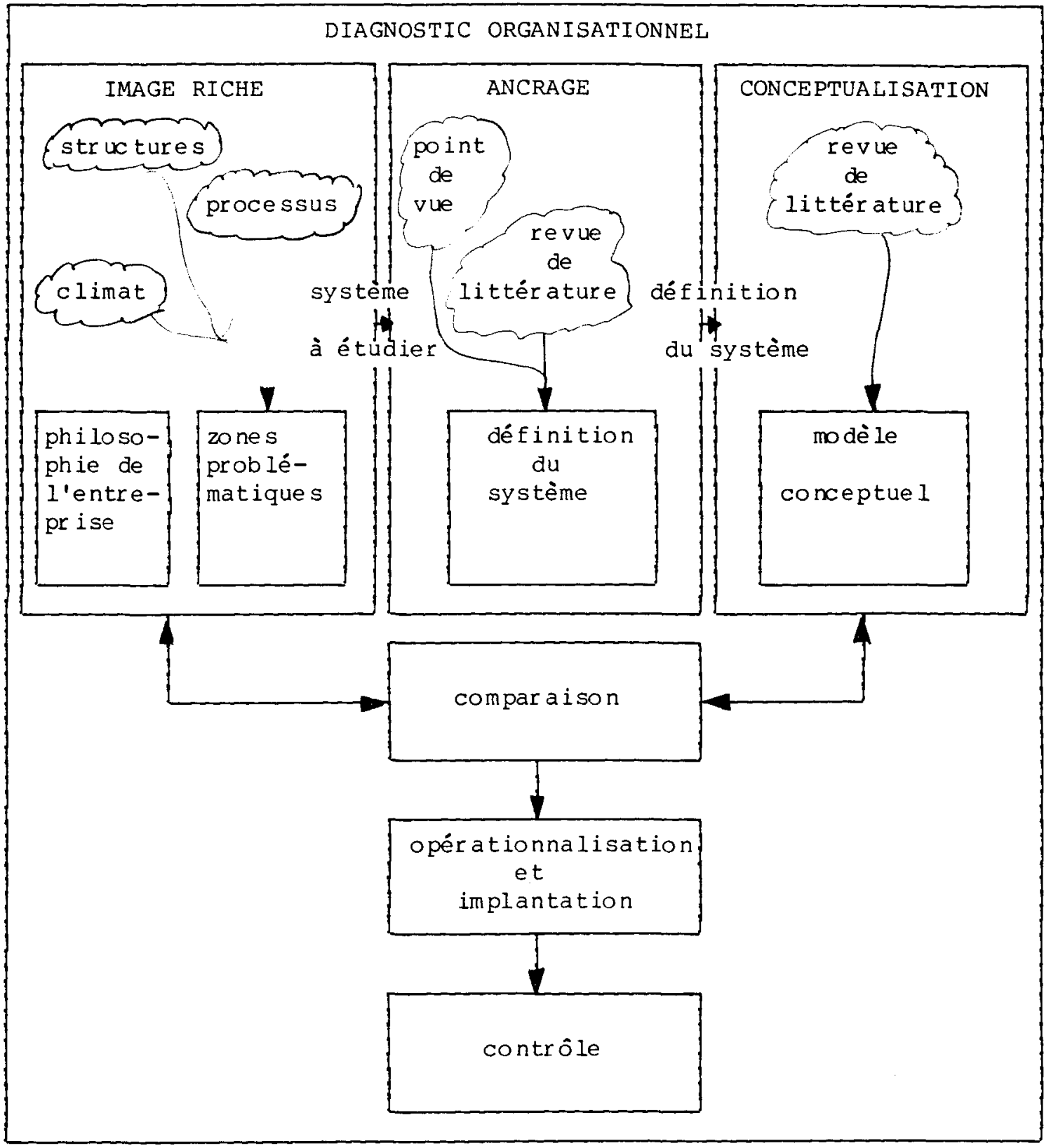

Figure 11.1 Méthodologie des systèmes souples de P.B. Checkland 


\section{1. ÉTAPES SUGGÉRÉES PAR LA MÉTHODE}

Avant de procéder à l'élaboration du mandat, le chercheur doit conquérir une certaine crédibilité auprès des personnes impliquées dans le diagnostic. "En effet, pour le laisser accéder aux aspect cachés des phénomènes rationnels, il faut que les sujets du diagnostic n'y voient pas de danger, et même qu'ils y voient une espérance de résultat bénéfique"( 28 ).

La figure 11.1 exposant la méthodologie du professeur Checkland, est tirée du document de paul prévost intitulé Le diagnostic-intervention: une approche systémique au diagnostic organisationnel et à la recherche-action, page 36 . Nous en décrivons ci-dessous les principales étapes.

\subsection{Mandat}

L'élaboration du mandat représente une étape importante pour mener à bien un diagnostic organisationnel. "Dans cette première activité, le conseiller ou le chercheur prend contact avec l'organisation et les principaux intervenants touchés par une problèmatique particulière alors que ceux-ci font de même avec celui-ci et sa méthodologie de travail".(27) une première difficulté pour le chercheur consiste donc à faire une nette distinction entre le poseur de problème, le décideur et le client: le poseur de problème (par exemple le directeur général) expose la problématique de l'organisation telle qu'il 
la perçoit. Quant au décideur (par exemple le conseil d'administration), il exerce le pouvoir de changer les choses, d'investir, etc. Le client de son côté, est le propriétaire du système qui fera lobjet de modifications. Nous pouvons cependant constater que cette difficulté s'estompe dans le cas de petites entreprises comme Imagem. Ces trois types d'acteurs se confondent nécessairement dans le cas où les trois propriétaires sont rencontrés pour l'élaboration du mandat,puisqu'ils sont à la fois poseurs de problèmes, décideurs et clients, à un degré bien sûr variable.

Une deuxième difficulté à surmonter concerne la définition de la problématique initiale. Dans ce cas ausi, les circonstances nous ont grandement aidés à minimiser les ambiguités. D'une part, nous avions déjà été approchés au printemps 1987, à titre de consultants chez Enjeu outaouais, pour participer à un groupe d'études dans l'entreprise Imagem. Ce groupe de travail, réunissant plusieurs intervenants gouvernementaux, devait procéder à une brève analyse de la situation chez Imagem qui vivait alors une crise financière aiguë. D'autre part, nous avons convoqué deux rencontres avec les trois propriétaires de l'entreprise avant d'élaborer le mandat final; ce qui a permis de faciliter un premier énoncé de la problématique. À cette étape, "il ne s'agit pas pour le chercheur d'analyser le problème mais plutôt de décrire la situation dans laquelle on a perçu un malaise". (27) 
Il faut ensuite définir clairement les rôles de chacun dans le processus de diagnostic organisationnel, de même que la méthodologie qui sera suivie par le chercheur. Dans le cas qui nous intéresse, nous avons pu constater l'importance pour les acteurs, de visualiser la démarche par un échéancier précis des activités. On était à même de comprendre la logique de la méthodologie, de même que l'importance relative, en temps, de chaque étape proposée. Il faut tout de même admettre que le temps leur a permis d'y voir plus clair.

Une discussion sérieuse sur les attentes de chacune des parties, facilite la poursuite du diagnostic. Dans notre cas, deux éléments sont prédominants: d'une part, les propriétaires de lentreprise souhaitaient notre intervention pour faire le point sur la situation réelle de l'entreprise et opérer des changements positifs et d'autre part, nous espérions leur participation active et leur collaboration dans un processus qui devait nous amener à l'élaboration de notre mémoire de maitrise. La négociation d'un cachet de $\$ 1500$ venait concrétiser l'entente entre les deux parties. Nous ouvrons encore ici une parenthèse sur les minces disponibilités financières dont dispose la petite entreprise, pour recourir à des ressources externes de consultation. La formule de troc pourrait s'avérer une solution intéressante dans ce contexte. 


\subsection{Image riche}

Checkland suggère à cette étape "que le chercheur identifie les principaux intervenants, partie prenante à la problématique, recueille le plus d'information possible sur leurs perspectives respectives et interprète les éléments structurants de sa description dans la réalité (structure, processus et climat) en fonction du type d'organisation ou du phénomène organisationnel qu'il aborde." (27).

Che z Imagem, cette façon d'aborder le diagnostic a permis d'émettre une première hypothèse suivant laquelle, les marchés en croissance compliquent la tâche quotidienne des gestionnaires des petites entreprises car ils les obligent à réévaluer régulièrement leur stratégie pour tenir compte d'un environnement turbulent et de leurs ressources limitées. Les gestionnaires éprouvent alors de la difficulté à évaluer clairement l'importance à accorder aux décisions opérationnelles, versus les décisions stratégiques.

La cueillette des données a été réalisée par la lecture de documents internes, de revues spécialisées et autres; par des entrevues avec les acteurs en place et quelques intervenants externes; et par l'observation des processus d'activités humaines. Les entrevues internes ont été menées auprès des trois propriétaires actuels et de trois pigistes au fait de la 
situation. Pour leur part, les entrevues externes ont été menées auprès du banquier de l'organisation, d'un représentant de l'organisme subventionneur, des dirigeants de trois agences de publicité de même qu'un responsable du centre du film et de la vidéo du gouvernement fédéral. Ces démarches externes ont permis d'améliorer la vision des propriétaires d'Imagem, en rapport avec la concurrence qu'ils sous-estimaient. Elles ont aussi permis d'en savoir plus long sur ce que certains intervenants externes pensaient de l'organisation et de mieux comprendre l'industrie de l'audiovisuel.

La cueiliette d'informations de nature comptable a nécessité notre implication soutenue sur une période d'une semaine, afin de faire le jour sur la situation financière de l'entreprise. Cette expérience nous a permis de comprendre le processus de controle de l'entreprise. par contre, elle impliquat l'obligation de prendre nos distances pour maintenir une certaine objectivité.

L'étude du climat organisationnel est loin d'être une mince affaire. Chez Imagem, la situation relativement tendue a nécessité une bonne dose de délicatesse de notre part, doublée de suffisament de fermeté pour obliger les acteurs à discuter des choses en profondeur. 
Nos démarches ont été facilitées par une bonne collaboration des acteurs, à tous les niveaux. De même, la présentation de l'image riche pour validation interne a été reconnue comme représentative de la situation actuelle et a d'abord amené les parties vers un consensus des nombreuses zones problématiques, puis de la zone problématique à solutionner.

Un dernier phé nomène que nous devons reconnaître en tant que chercheur, est l'effet dynamique et non statique, qu'engendre tout diagnostic organisationnel dès le début de la démarche. Selon Collerette et Delisle, dans leur volume sur le changement planifié: "Comprendre le changement, c'est tenter d'expliquer un processus continu qui se situe au centre de la réalité des organismes vivants et qu'il est difficile d'isoler entre deux points."(6)

\subsection{Ancrage}

"La phase d'ancrage détermine la perspective sous laquelle la problématique sera étudiée et fixe les paramètres essentiels du ou des système(s) d'activités humaines qui serviront de référence pour étudier la situation problématique particulière qui a été sélectionnée". (27) À cette étape, il nous faut distinguer les symptômes des problèmes si l'on veut prendre une décision efficace et appropriée. À cet effet, les 
auteurs Landry et Maloin démontrent clairement quatre types de danger auxquels fait face le chercheur: "I'ignorance de la loi de la situation", qui privilégie non seulement l'étude des apparences externes du phénomène, mais aussi l'ancrage sur lequel elles reposent; "l'erreur de s'en tenir aux faits", qui peuvent camoufler d'autres faits encore plus pertinents; "le danger de confondre les situations uniques et les situations génériques", ces dernières étant le signe d'un désordre fondamental dont ce n'est pas le cas pour les premières; et " le danger d'épouser trop étroitement la perspective d'un seul acteur ou groupe d'acteurs" (17). Chez Imagem par exemple, le fait que l'entreprise n'ait pas encore réussi à se tailler une place évidente dans le marché; que les gestionnaires soient insatisfaits de la tangente de diversification prise jusqu'à ce jour; que l'on dénote une certaine forme d'indépendance entre les différents systèmes d'activités; que les ressources soient souvent mal utilisées, etc. permet d'envisager comme probable, un manque flagrant au niveau du système de management stratégique.

Cette première réflexion nous a ensuite amenés à utiliser la grille d'analyse de Joseph Chicha, afin d'évaluer plus en profondeur la stratégie actuelle de l'entreprise. On retrouve entre autres le critère de cohérence, de loin le plus important à divers niveaux: "la complémentarité des composantes du contenu de la stratégie, la conformité de la 
stratégie avec les choix préalables, l'adéquation entre la stratégie et les ressources de l'entreprise et la prise en compte des facteurs environnementaux dans l'évaluation des alternatives et des choix". (5) Parmi les cinq autres critères proposés par l'auteur, nous avons retenu les plus pertinents, soit: "l'intégration stratégique, exprimant la mesure dans laquelle la stratégie a intégré les fonctions de l'entreprise; et l'étapisme stratégique, correspondant à l'échelonnement planifié sur une période de temps plus ou moins longue, des grandes manoeuvres de la stratégie". (5)

Cette évaluation plus précise de la situation problématique, appuyée d'une revue de littérature exhaustive, nous a graduellement orientés vers une définition de plus en plus claire du point d'ancrage ou en d'autres mots, du système d'activités à améliorer. Il s'avéra, après quelques ajustements avec les acteurs, que ce point d'ancrage correspondait effectivement à leurs principales préoccupations.

Précisons finalement, que toutes ces étapes de la méthodologie constituent un processus complet d'induction, décrit dans un volume traitant des méthodes de recherche en sciences sociales comme: "une opération qui consiste à dégager des inférences à partir de certaines observations spécifiques pour constituer une règle plus générale". A l'étape suivante de la conceptualisation, nous serons plutot entraînés dans un 
processus de déduction, décrit comme:" un processus de raisonnement logique gràce auquel on tire des conclusions à partir de prémisses. Il sert à dégager des prédictions d'une théorie".

\subsection{Conceptualisation}

Les principales bases de la conceptualisation se situent au niveau de I'ancrage et d'une revue exhaustive de littérature. Cette étape implique la construction d'un modèle qui prévoit toutes les activités nécessaires pour se conformer à la définition du système. "Le modèle conceptuel est une élaboration aussi objective que possible qui n'a pas de prétention idéalisante, ni normative et ce n'est pas un modèle général" (27)

Ce sont les écrits respectifs de messieurs chicha et Bamberger qui nous ont le plus sérieusement influencés dans l'élaboration du modèle conceptuel présenté au chapitre IX. Il a cependant fallu ramener leur vision du processus de management stratégique à une représentation simplifiée pour s'en tenir aux activités essentielles d'un tel processus.

\subsection{Comparaison}

Cette étape consiste à comparer la situation problématique de l'entreprise avec le modèle conceptuel, dans le but 
d'identifier les changements potentiels. Dans le cas où la situation problématique de l'entreprise est non structurée comme chez Imagem, "le modèle conceptuel constituera tout au plus un cadre de référence cohérent et formel capable d'organiser un débat structuré entre les intervenants sur la situation problématique" (27)

\subsection{Opérationnalisation, implantation et contrôle}

Parmi les changements potentiels identifiés lors de l'étape précédente, certains s'avèrent fondamentaux et d'autres mineurs. Les intervenants au dossier choisiront les changements désirables et réalisables. Le chercheur doit retenir à ce niveau, qu'il est normal que les intervenants soient conservateurs dans leur choix et ainsi, il ne doit pas leur imposer des changements élégants. "À la limite, il vaut mieux alors semer un germe de changement qui amènera par la suite les intervenants à demander eux-mêmes d'autres changements plus substentiels" (27). Chez Imagem, il nous apparaît évident après coup que bien que certains changements aient été reconnus comme désirables dans un premier temps, ils peuvent être ramenés au second rang des priorités quelques semaines plus tard.

Ajoutons que le chercheur doit plus que jamais jouer son rôle de facilitateur du changement et d'éducateur. Des 
changements importants peuvent nécessiter la réutilisation de la méthode de diagnostic-intervention en tout ou en partie afin d'élaborer une stratégie qui permet d'atteindre les objectifs de l'étape d'implantation. C'est d'ailleurs une réalité chez Imagem, ò̀ l'intervention du chercheur au niveau de 1'implantation s'est échelonnée sur une période de cinq à six semaines; de façon ponctuelie. Ce qui constitue d'ailleurs l'étape décisive et finale pour le chercheur, du long processus d'apprentissage rattaché au diagnostic-intervention.

\section{DIAGNOSTIC-INTERVENTION, UNE DÉMARCHE SCIENTIFIQUE?}

Les tenants du diagnostic-intervention se voient régulièrement confrontés à un débat épistémologique en science de la gestion. La démarche scientifique est vue "comme une démarche expérimentale, réductionniste et causaliste dont la validation (le contrôle) est assurée par la notion d'évidence que justifie une exigence de répétitivité et un effort de réfutation". (27)

La méthode scientifique est parfois qualifiée comme analytique, classique, cartésienne ou traditionnelle. "C'est une logique fondée sur la déduction allant du simple au complexe... [on y considère que] les éléments simples se comportent de la même manière quand ils sont isolés et quand ils sont regroupés: on ne tient pas compte des interactions". (16) 
Ce qui apparait comme peu rigoureux dans la méthode scientifique traditionnelle pour les tenants du diagnosticintervention, est le fait que les paradigmes, sur lesquels repose la méthode scientifique, échappent à la sévérité du contrôle de la dite méthode. Ces paradigmes ne sont pas validables par la méthode.

Par opposition à cette pratique faible quant à sa rigueur, les approches systémiques (en l'occurence l'approche des systèmes souples de Checkland) sont fières d'exposer leur propre démarche de validation. Elle se fait à quatre niveaux:

Dès la première étape de la méthodologie, l'élaboration de l'image riche est validée par consensus avec les intervenants. Ensuite, la définition du système jugé pertinent sera validée dans sa cohérence interne, par l'utilisation d'un langage commun et par le respect d'un cadre précis. Pour sa part, l'ancrage sera validé dans sa cohérence externe et ses relations avec des cadres idéologiques ou théoriques plus larges, par une réflexion autour du choix du point de vue. Le choix se fera par consensus ou de façon dialectique, selon que le débat engendre ou non des confrontations entre les intervenants. La troisième étape de la méthodologie de Checkland, (la conceptualisation) respecte la même procédure de validation que le point d'ancrage. Finalement, la problématique et la 
conceptualisation sont soumis à un processus de validation dialectique. On les compare pour mieux en dégager les éléments incongrus.

Les auteurs de telles réflexions conclueront finalement que la somme des expériences et des cas qui seront cumulés par l'utilisation de la méthode checkland "pourront prétendre éventuellement à contribuer non seulement à l'accroissement du savoir-faire et du savoir-être, mais aussi à l'accroissement du savoir cognitif relié au comportement des systèmes d'activités humaines". (27)

D'autres auteurs d'ajouter: "Le concept de systémisme ne doit pas être jugé uniquement par rapport à sa scientificité et à sa valeur prédictive mais bien par rapport à son efficacité pour fournir des explications dans un champ complexe" (18). 


\begin{abstract}
La critique méthodologique constitue un effort intéressant pour améliorer notre compréhension de la méthode du professeur Checkland, à partir d'un cas pratique.
\end{abstract}

Ainsi, nous retiendrons que s'il n'est pas toujours
facile pour le chercheur de se ramener aux volontés des
dirigeants, (la tentation étant parfois forte de proposer des
solutions qui collent à sa façon de voir les choses) la
méthode du professeur checkland ramène régulièrement le
chercheur à une intervention par consensus.


XI I

L'IMPACT DU DIAGNOSTIC CHEZ IMAgEM 
Après s'être questionnés sur les différentes facettes de la méthodologie privilégiée, à savoir son efficacité dans la résolution de problèmes complexes dans les petites et moyennes organisations et sa conformité avec la recherche action d'une part et les études scientifiques systémiques reconnues dans le monde de la recherche d'autre part; nous devons réfléchir sur l'impact réel du diagnostic sur l'entreprise Imagem, sur ses propriétaires et son personnel, deux mois après notre intervention.

Dans un premier temps, nous exposerons surtout les effets positifs de notre intervention sur le comportement des propriétaires: l'effet de déstabilisation du départ, leur capacité de reprise en mains et leurs actions concrètes. Nous nous attardons par la suite aux effets sur l'entreprise en e 1 le-même.

Dans un deuxième temps, nous réfléchirons sur les objectifs qui ne sont pas encore atteints et sur les raisons qui peuvent expliquer cette réalité. 
Voyons d'abord l'impact du diagnostic sur le comportement des propriétaires de l'entreprise. Si au début, le diagnostic a déstabilisé les trois propriétaires-dirigeants de l'entreprise, on constate maintenant qu'ils ont repris de l'assurance et pris conscience de leurs propres limites et capacités; "le processus de changement vécu par des personnes serait marqué dans un premier temps par l'abandon des comportements et attitudes habituelles. Suivrait ensuite une période marquée par des comportements ou attitudes plus ou moins instables, diffus, pour déboucher sur l'acquisition de nouveaux comportements ou attitudes" ( 6 ). Ainsi, le départ de monsieur Gendron, responsable de l'administration jusqu'en novembre 1987, a nécessité, avec notre aide, la reprise en main de la situation par les deux associés en place, en aussi peu que deux à trois semaines. Nous sentons renaitre une certaine fierté d'être associés à l'entreprise Imagem depuis cette période. Ajoutons aussi que les propriétaires-dirigeants réalisent de plus en plus l'importance de s'adjoindre du personnel compétent pour améliorer leurs chances de survie: "la profession de dirigeant d'entreprise est aujourd'hui devenue une réalité complexe qui nécessite, en plus du recours à des ressources extérieures compétentes, des capacités de planification et d'organisation de la part de la personne qui s'y implique ainsi qu'une préparation de plus en plus spécialisée de cette personne dans l'exercice de la profession qu'est devenue la gestion d'une entreprise"(13). Nous constatons que l'entreprise s'engage 
vers le stade de l'adolescence. Dans leur recherche de personnel, les dirigeants ont ainsi déniché une ressource comptable externe, une ressource externe pour mener à bien la mise en marché de leur projet d'auto - construction et de nouveaux pigistes pour la conception et la production audiovisuelle. Par-dessus tout, notons leur confiance plus que doublée dans un avenir intéressant, bien qu'exigeant, en terme d'énergie à y consacrer pour réussir.

Analysons maintenant l'impact du diagnostic sur l'entreprise elle-même. En premier lieu, le diagnostic a permis de faire la lumière sur la situation réelle de l'entreprise et d'évaluer les solutions qui permettent d'y opérer un changement favorable pour l'entreprise et ses propriétaires. On a ainsi procédé au réalignement de la stratégie de l'entreprise et à de nombreux réajustements de mise en oeuvre stratégique. Deux mois après la fin du diagnostic, nous pouvons avancer qu'environ $50 \%$ du plan opérationnel a été réalisé. C'est plus que satisfaisant pour une jeune entreprise à laquelle on propose de nombreux réajustements, parfois radicaux. Dans un premier temps, les propriétaires de l'entreprise se sont surtout attardés à redéfinir leur mission et leurs objectifs généraux. Par la suite, ils se sont attaqués plus intensivement au plan opérationne 1. Sur le plan financier, $80 \%$ des réajustements de mise en oeuvre stratégique ont été entrepris; entre autres, la préparation d'états financiers 
prévisionnels, la finalisation du dossier de l'assurance-feu, le recherche de subventions possibles et la rencontre du banquier pour renégocier la dette. Au plan du personnel, ils ont intensifié les efforts pour trouver des ressources compétentes qui répondent aux besoins de l'entreprise. Au niveau du marketing, les dirigeants de l'entreprise tentent de lancer leur nouveau projet d'auto - construction par des contacts sérieux et la préparation d'un dossier de pré-faisabilité, assistés d'une ressource externe compétente. De plus, leur approche auprès de la clientèle cible s'oriente de plus en plus vers un style de consultant en audiovisuel et non de vendeur de produits et services audiovisuels. Finalement, au niveau de la production, ils développent une expertise de plus en plus sérieuse, dans la réalisation de productions de type "broadcast" et dans la réalisation de diaporamas. Cette expertise est le fruit d'un apprentissage par essais et erreurs, combiné à une solide formation académique.

Dans un autre ordre d'idée, nous pouvons constater, avec une certaine réserve, I'augmentation de la visibilité de l'entreprise. Le temps fait bien les choses et les efforts antérieurs des propriétaires n'ont pas été vains. Cependant, il est aussi indéniable que notre visite chez les intervenants externes de l'entreprise, a convaincu plusieurs d'entre eux, du sérieux et de la perspicacité des dirigeants d'Imagem. Ainsi, le banquier a semblé apprécier une telle démarche 
puisqu'il a accepté en bloc la restructuration de la dette. De plus, le responsable du centre du film et de la vidéo du gouvernement fédéral, M. Robesco, a approché l'entreprise dès janvier 1988 pour s'inscrire à titre de fournisseur potentiel de son organisme; et il est permis de croire que les contacts effectués auprès de certaines agences de publicité outaouaises puissent servir de tremplin à une représentation plus partisane des services et produits de l'entreprise Imagem.

Dans le prochain paragraphe, nous réfléchirons sur les réajustements de mise en oeuvre stratégique qui tardent à se réaliser puis nous tenterons d'en élucider les causes. Au plan de l'organisation de l'entreprise et du personnel, c'est surtout l'amélioration des structures qui fait défaut; par exemple: les contrats types d'embauche de pigistes, les échelles salariales, les définitions de tâches et responsabilités et la préparation de dossiers de pigistes. Au - plan de la production, la formation du comité de contróle de la qualité tarde à se réaliser. Au plan du marketing, on note un certain ralentissement dans les efforts de relations publiques pour les produits et services de base de l'entreprise. De plus, la production d'un vidéo promotionnel et les améliorations au dossier de présentation d'Imagem ne seront pas réalisées avant quelques mois; ce qui était cependant prévu dans le calendrier des activités. 
Finalement, sur le plan financier, seulement 10 à $20 \%$ des objectifs de départ tardent à se concrétiser. on doit cependant redoubler d'efforts pour assurer la disposition des équipements inutiles encore en main d'une valeur de $\$ 8500$ et poursuivre la perception assidue des comptes à recevoir, rattachés au projet Vidéotour qui traine encore un peu. Selon nous, les causes qui expliquent la réalisation partielle du plan opérationnel sont nombreuses. Premièrement, Imagem manque de ressources permanentes. Ainsi, le départ d'un des trois gestionnaires n'a pas encore été comblé à ce stade de la réorganisation et cela entraine des répercussions, surtout au plan marketing et financier. Deuxièmement, il nous apparait évident que les deux gestionnaires en place considèrent certaines actions, surtout rattachées aux éléments structurants, comme nécessaires mais non prioritaires dans l'état actuel de la situation. Troisièmement, nous dénotons une certaine inertie dans la tâche à accomplir; les gestionnaires se sentent parfois décontenancés et ne savent alors plus par quel bout commencer. C'est le cas pour le plan de production et de personnel. Finalement, un certain individualisme dénoncé lors du diagnostic, continue de se maintenir dans l'air. Ainsi, les deux gestionnaires en place et le troisième gestionnaire qui n'est pas totalement hors circuit, évitent les grandes remises en question sur les définitions de tâches et les responsabilités. 
Pour conclure sur l'impact du diagnostic organisationnel réalisé chez Imagem, nous ne pouvons que constater qu'il a été bénéfique pour l'entreprise et ses propriétaires dirigeants. Il est cependant indéniable que le travail du chercheur ne consistait pas uniquement à fournir un diagnostic clair, mais aussi un appui soutenu pendant quelques semaines du plan opérationnel. Ce suivi tend cependant à se résorber petit à petit, afin de laisser les acteurs réaprivoiser leur indépendance pour jouer un rôle de premier plan dans le système de management stratégique qu'ils ont contribué à élaborer. 
UNE RÉFLEXION SUR L' INTERVENTION GOUVERNEMENTALE AUPRÈS DES PETITES ENTREPRISES 
Dans les quelques pages qui vont suivre, nous traiterons de l'intervention gouvernementale auprès des petites entreprises en phase de démarrage. Notre réflexion se divise en cinq thèmes. D'abord, nous rappelerons au lecteur les besoins des petites entreprises en phase de démarrage et nous tenterons de faire le portrait de son propriétaire. Ensuite, nous nous questionnerons sur le rôle actuel des nombreux intervenants gouvernementaux préoccupés par l'entrepreneurship, et sur le rôle qu'ils devraient jouer pour améliorer le service à la clientèle. Nous tenterons ensuite de définir l'organisme d'aide idéal, pour finalement focaliser sur les processus de consultation et le consultant dans l'entreprise.

\section{BESOINS DE LA PETITE ENTREPRISE EN PHASE DE DÉMARRAGE ET PORTRAIT DE SON (SES) PROPRIÉTAIRE (S)}

Rappelons-nous que l'entreprise se situe encore en phase de démarrage au cours de ses deux premières années d'opération. On peut donc tenter de faire une différence entre les besoins de pré-démarrage (un an et moins avant l'ouverture de l'entreprise) et les besoins de démarrage. Les besoins spécifiques aux petites entreprises sont de deux types: d'abord des ressources humaines compétentes en gestion, ensuite des ressources financières. Ainsi, les besoins de pré-démarrage se concentrent essentiellement sur le soutien dans: l'étude de marché, l'étude de localisation, l'étude 
technique, la préparation d'états financiers prévisionnels, l'élaboration de campagnes publicitaires et la préparation des demandes d'aide financière. Par contre, les besoins en phase de démarrage bifurquent quelque peu vers le soutien comptable et marketing, les conseils fiscaux et légaux mineurs et l'analyse globale de la situation, bref, une école pratique de formation de l'entrepreneur, un suivi rigoureux.

Nous devons ausi nous demander à quel type d'individus s'adresseront ces programmes. Notre expérience en démarrage d'entreprises nous amène à supposer au sujet des entrepreneurs qui auront le plus besoin de ces services: qu'ils sont rarement diplómés ou expérimentés en administration des affaires, qu'ils connaissent peu les problèmes auxquels ils devront faire face et se sentent donc peu craintifs de s'y aventurer. Nous constatons aussi qu'ils ont généralement une bonne connaissance du domaine dans lequel ils veulent se - Iancer; ce qui contribue à augmenter leur assurance dans la réalisation du projet. Finalement, n'oublions pas que les promoteurs désirent souvent créer leur propre emploi à cause d'insatisfactions relatives au marché actuel de l'emploi. 


\section{RÔLE ACTUEL DES INTERVENANTS GOUVERNEMENTAUX EN ENTREPRENEURSHIP}

En nous arrêtant un peu pour dresser une liste non exhaustive des intervenants gouvernementaux en entrepreneurship, quel fut notre étonnement de pouvoir en énumérer une quinzaine. Selon Yvon Gasse, "La création d'emplois, 1'innovation et la faiblesse en matière de gestion des entrepreneurs sont des raisons parmi d'autres qui ont amené cet intérêt plus prononcé... à développer des entrepreneurs" (14). Entre autres intervenants, on retrouve: les municipalités; les corporations locales, régionales et nationales de développement économique; les ministères à vocation économique; les institutions d'enseignement et les autres organismes. Par exemple, dans la région de l'outaouais on compte la Société d'aménagement de l'outaouais (SAO), la Société d'expansion économique de Gatineau (SEEGA), la Banque fédérale de développement économique (BFD), le ministère de I'Industrie et du commerce (MIC), les deux groupes de soutien aux initiatives jeunesse de 1'outaouas (GSIJ), l'office de planification et de développement du québec (OPDQ), l'université du Québec à Hull (UQAH), le Collège de l'outaouais, les départements d'éducation des adultes des commissions scolaires, le centre de recherche industrielle du Québec, le secrétariat régional de la concertation de 1'outaouais (SRCO), Emploi et Immigration Canada par son pro- 
gramme de protection de l'emploi dans les entreprises canadiennes (CAMO) et bien sûr, les municipalités. Une telle constatation nous permet ausi de nous demander si les services d'autant d'intervenants sont au moins utiles pour les entrepreneurs eux-mêmes. Nous pouvons distinguer quatre tendances majeures dans les activités privilégiées par ces organismes: 1 a promotion de l'entrepreneurship, la formation, l'aide financière et l'aide technique. Afin d'y voir plus clair, nous avons ainsi jugé bon de représenter les intervenants et leurs activités à la figure 13.1. Une activité se rattache à l'organisme seulement si elle représente du temps ou des efforts considérables pour celui-ci.

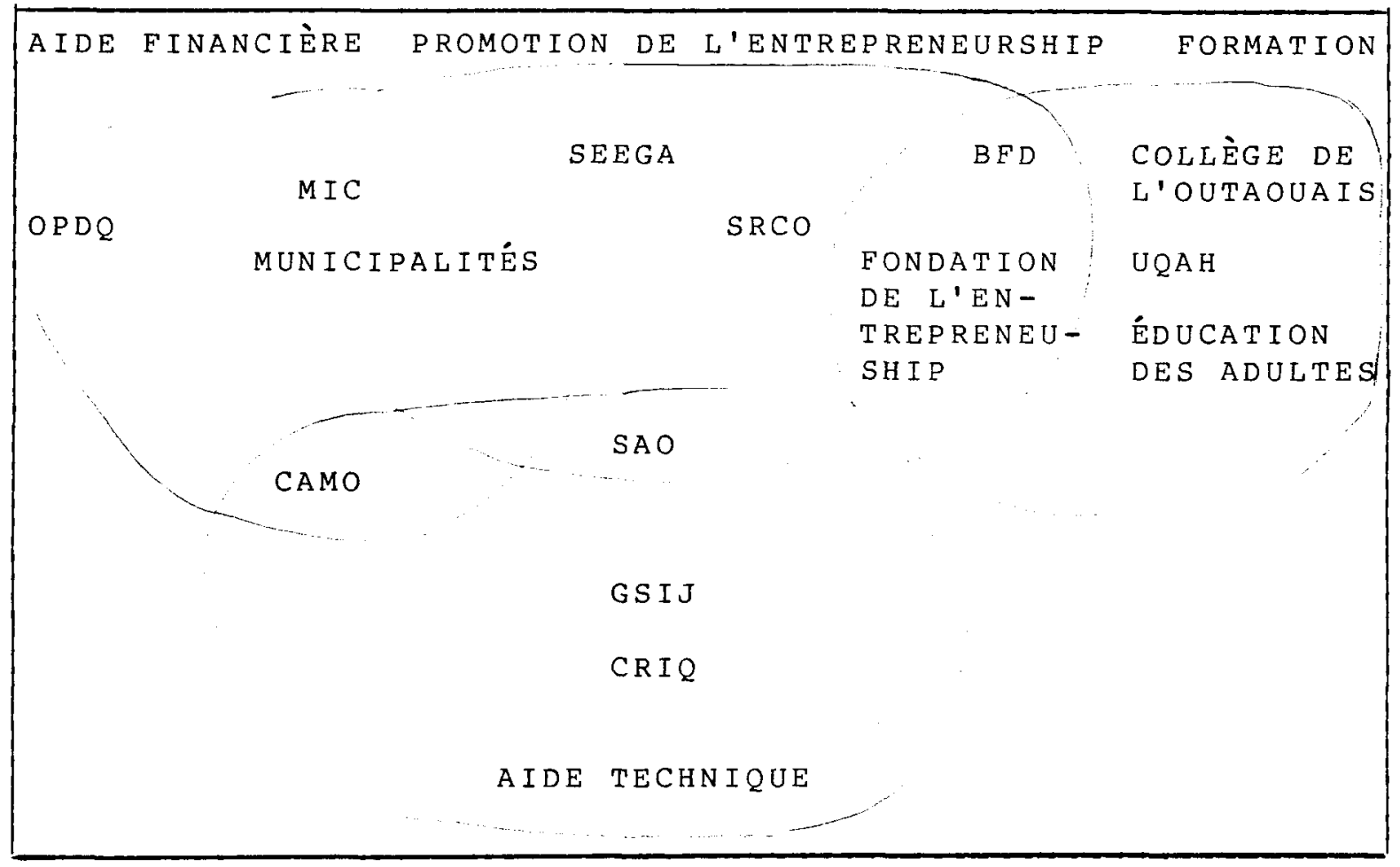

FIGURE 13.1 Les intervenants en entrepreneurship de l'outaouais et leurs activités 
on constate à l'analyse de la figure 13.1, que plusieurs intervenants dépensent des sommes importantes d'énergie à faire la promotion de l'entrepreneurship, soit près de $50 \%$ d'entre eux. D'autres organismes se préoccupent un peu plus de la formation des futurs ou actuels entrepreneurs, soit environ 25\%. Finalement, 1'aide financière aux entrepreneurs représente une activité principale chez $25 \%$ des organismes et pour une même proportion au niveau de l'aide technique.

Selon nous, cette situation reflète des problèmes majeurs. D'abord les intervenants en entrepreneurship consacrent beaucoup trop d'énergie à en parler, plutôt que d'agir auprès des entrepreneurs. Ensuite, la bureaucratie amplifie cette situation d'inaction. Prenons comme exemple le MIC. Ses intentions sont fort louables mais il est plutôt mal perçu dans la communaté des entrepreneurs. Le personnel y passe. un temps considérable à faire de la promotion, à analyser les dossiers qui lui sont présentés et à faire le suivi des dossiers. Notons ici que l'expression est fort bien choisie: on y suit les dossiers et non les entreprises en opération. pour plusieurs organismes gouvernementaux, suivre un dossier signifie s'assurer que les normes d'admissibilité sont respectées, que tous les documents sont au dossier, s'assurer aussi d'obtenir des copies des états financiers périodiques, et de faire des visites éclair dans l'entreprise pour se sentir à l'aise d'en prendre note dans le dossier. Selon 
nous, faire le suivi d'une entreprise démarrée ne ressemble en rien à cela, comme il a été expliqué précédemment.

En outre, bien que certains intervenants s'impliquent dans l'aide technique aux entreprises, les services qu'ils offrent sont encore trop fragmentaires. Par exemple, la sAo a surtout concentré ses études de marché dans des projets d'envergure de type touristique. Pour sa part le CRIQ concentre ses études sur les nouveaux produits ou nouvelies techniques de production. Les programmes CAMO aident surtout les entreprises en difficulté de plus de quinze employés, dans l'élaboration de diagnostic et solutions applicables. Finalement, les GSIJ concentrent leur aide technique auprès des entrepreneurs de moins de trente ans, en phase de pré-démarrage, faisant du suivi de façon plutôt limitée. De plus, il est indéniable que leurs services de pré-démarrage auraient avantage à être améliorés en terme de quantité et de qualité par des équipes de travail multidisciplinaires. Le portrait que l'on retrouve chez la majorité des quatre-vingt-cing groupes du Québec est sensiblement le même: un comptable et un secrétaire; un diplômé en marketing et un secrétaire; un diplômé en personnel et un diplômé en marketing, etc. Enfin, quelle que soit la combinaison de deux personnes, il restera toujours un manque de personnel pour combler tous les besoins des entrepreneurs de façon satisfaisante. 


\section{ROLLE À PRIVILÉGIER CHEZ LES INTERVENANTS EN ENTREPRENEURSHIP}

Inévitablement, il faut admettre que les entrepreneurs souffrent d'un manque de soutien technique de pré-démarrage, de suivi de leurs activités d'entreprise et de formation en gestion sur une base individuelle. Les intervenants qui visent à la fois la promotion de l'entrepreneurship et l'aide technique, s'orientent souvent vers un cul-de-sac; en ce sens qu'ils sont souvent prêts à sacrifier les activités plus techniques, aux activités promotionnelles. C'est pourquoi nous considérons que les organismes de promotion, ne devraient pas être trop directement reliés aux organismes d'aide technique, si on veut assurer des services adéquats aux entrepreneurs. Finalement, on doit cesser de penser que les structures physiques représentent la solution idéale aux problèmes des petites et moyennes entreprises. Nous pensons ici aux - incubateurs d'entreprises d'une. façon stricte. Comme nous avons pu le constater lors de recherches personnelles sur ce phénomène, ce ne sont pas les locaux qui manquent le plus aux petites entreprises; ce sont les ressources humaines. C'est donc à ce niveau que les gouvernements doivent intervenir. Dans la prochaine section, nous tenterons de définir l'organisme idéal... 
Avant de procéder à cette brève description, nous devons nous questionner sur la clientèle cible qui pourrait être touchée par de telles mesures. On pense bien sûr, aux entreprises manufacturières et de transformation; aux projets agro-alimentaires; aux entreprises qui s'intègrent dans des marchés prometteurs et en croissance telle qu'Imagem; aux entreprises où la compétition est faible même si elles font partie du secteur tertiaire.

\section{UN ORGANISME IDÉAL D'AIDE À L'ENTREPRENEUR}

$$
\text { L'organisme idéal d'aide à l'entrepreneur devrait }
$$

nécessairement offrir les services les plus complets de pré-démarrage et de suivi aux entreprises ciblées dans le paragraphe précédent. Pour ce faire, on doit regrouper et non disséminer les ressources humaines et financières. Il ne s'agit donc pas d'installer un bureau de deux cents fonctionnaires à Québec, pour aider les entrepreneurs de la province. Il s'agit plutôt de respecter les populations en périphérie, sans se contenter de leur donner l'illusion qu'ils ont des services. Par exemple, pour une région comme l'outaouais, sept personnes regroupées sous un même organisme et quelques contractuels pourraient faire un excellent travail, le tout appuyé d'une répartition adéquate des ressources. On pourrait ainsi y retrouver: deux gé néralistes en gestion des pmo qui suivraient les dossiers de $A$ à $Z$, deux 
diplômés en marketing, un diplômé pour l'analyse technique et financière, un secrétaire et un directeur général affecté au développement des services et à la bonne marche des opérations. Il est juste de penser que cet organisme pourrait ainsi mener une cinquantaine de dossiers sérieux à chaque année pour des petites entreprises outaouaises.

Cet organisme devrait s'adresser à des entrepreneurs de tout âge. Sa mission sous-entendue consisterait à former les entrepreneurs sur le terrain, afin de renforcer cet élément de plus en plus ancré dans la culture québécoise qu'est l'entrepreneurship.

Finalement, comme il a déjà été exprimé précédemment, cet organisme ne devrait pas sombrer dans la bureaucratie et les efforts à outrance de promotion, représentation et autres activités qui privilégient la discussion à l'action. 


\title{
5. POUR UNE MEILLEURE INTERVENTION DES CONSULTANTS
}

GOUVERNEMENTAUX DANS LES PETITES ENTREPRISES

\begin{abstract}
Nous tenterons ici d'exposer les éléments primordiaux à considérer lors de l'intervention des consultants gouvernementaux dans la petite entreprise.
\end{abstract}

Il faut nécessairement gagner la confiance de l'entrepreneur si on ne veut pas être considéré comme un intru. Cette confiance s'acquiert bien sûr par le temps mais aussi, en évitant de vouloir structurer l'entreprise à l'image de lorganisation gouvernementale, en maintenant un contact régulier avec l'entrepreneur et en lui démontrant par des gestes concrets qu'on est toujours prêt à lui ouvrir d'autres portes en cas de difficultés.

Il faut aussi impliquer l'entrepreneur dans toute forme d'intervention, ne pas agir en conquérant. L'entrepreneur doit prendre conscience qu'il a un rôle à jouer dans la démarche.

Les mandats spéciaux doivent être clairement définis afin d'éviter les insatisfactions de part et d'autres.

Le consultant doit se rappeler que la démarche doit être systémique; que dans un processus d'identification de problèmes, les individus se représentent différemment la 
réalité et les problèmes qui s'y rattachent; que pour chaque vision du problème correspond un nombre de solutions admissibles et un nombre de solutions inadmissibles. Le défi du consultant consiste donc à proposer des solutions qui permettent de faire consensus entre les acteurs de l'entreprise.

Finalement, le consultant gouvernemental ne doit pas hésiter à recourir à des ressources externes dans le cas où les problèmes sont complexes et qu'il se sent dans 1'incapacité d'aider l'entrepreneur à les résoudre. 


En partant de l'hypothèse que la petite entreprise
requiert avant tout l'appui de ressources humaines
compétentes, il devient relativement simple de définir
l'organisme gouvernemental idéal d'aide à la petite
entreprise. Encore faut-il savoir quels services sont déjà
offerts dans ce milieu. Ainsi, nous avons pu constater que
des énergies considérables sont englouties dans les activités
de promotion; comparativement aux activités de formation, aide
technique et aide financière aux entreprises. Nous émettions
alors l'opinion qu'il devient de moins en moins justifié de
parler d'entrepreneurship; il faut agir.


CONCLUSION 
Les petites entreprises présentent des caractéristiques distinctes de la moyenne et de la grande entreprise. Les auteurs Robidoux, Lorrain et Dussaul, perrealt et Dell'Aniello nous exposent de nombreuses caractéristiques propres à la petite entreprise. Ils parlent entre autres du fait: que la petite entreprise est le prolongement de son propriétaire; que ce propriétaire qualifié d'homme-orchestre n'est pas porté à discuter avec d'autres de ses idées; qu'il concentre ses efforts a niveau de la direction, de la production et de la vente de ses produits (les domaines qu'il connait le mieux); qu'il fait peu de planification et que le système comptable de l'entreprise est souvent rudimentaire. Pour notre part, nous constatons aussi que les dirigeants des petites entreprises sont souvent inconscients de leurs besoins au niveau des ressources humaines en finance, marketing ou autre et qu'ils croient pouvoir tout organiser seuls, sans l'aide de personnel compétent.

La petite entreprise, tout comme la moyenne et la grande entreprise, a besoin d'une gestion équilibrée où on tient compte de toutes les forces en présence pour prendre de bonnes décisions. Nous considérons donc que c'est au niveau des ressources humaines de gestion que se retrouve la plus grande vulnérabilité des petites entreprises. Elles vivent un manque de ressources humaines compétentes et diversifiés, en gestion: phénomène qui ne se rencontre pas aussi souvent dans les deux autres types d'entreprise. 
De plus, cette vulnérabilité s'accentue par un second problème d'importance: la faible marge de manoeuvre financière de l'entreprise et de son ou ses propriétaire(s).

Considérant cette réalité, comment la petite entreprise peut-elle survivre aux premières années d'exploitation?

Nous osons croire que l'intervention gouvernementale peut faire beaucoup pour l'aider. Il faut cependant prendre conscience des besoins actuels des petites entreprises. Les besoins en pré-démarrage se concentrent essentiellement sur le soutien dans la préparation du plan d'affaire et des dossiers de demande d'aide financière. Pour leur part, les besoins en phase de démarrage (an un et deux) s'orientent plutôt vers le soutien marketing et comptable, les conseils de base sur le plan fiscal et légal, la stratégie d'entreprise et l'analyse globale de la situation: en bref, une école pratique de formation de l'entrepreneur, un suivi rigoureux.

Les intervenants gouvernementaux doivent aussi réaliser que leurs efforts demeurent beaucoup trop orientés sur la promotion et le développement de l'entrepreneurship au sens large. Il nous apparait essentiel de détourner une bonne partie des ressources humaines et financières actuelles de 
nombreux organismes vers des activités qui permettraient de répondre plus adéquatement aux besoins des petites entreprises, exprimés plus haut.

Dans le prochain paragraphe nous tenterons de faire un parallèle entre la problématique générale des pME et celle de I'entreprise Imagem, à partir de l'hypothèse que nous avons émise avant de réaliser le diagnostic organisationnel.

A ce stade-ci de notre recherche, nous constatons que l'hypothèse en question est valable sans pouvoir prétendre tout expliquer. Ainsi nous pouvons comprendre que les dirigeants d'Imagem, jusqu'à ce jour centrés sur la production, éprouvent une certaine difficulté à passer des décisions opérationnelles, aux décisions stratégiques lorsque les turbulences de l'environnement I'exigent. Cependant, cela n'explique pas leur difficulté d'analyser la santé de leur entreprise. Nous ne pouvons alors que constater leur manque de ressources humaines compétentes en gestion. Pouvons-nous imaginer pendant un instant I'histoire de cette même entreprise, si elle avait bénéficié dès son ouverture de ressources compétentes en gestion? Ce serait sûrement fort différent.

par surcroît, Imagem vit une certaine instabilité financière qui ne lui permet pas d'opérer des revirements majeurs de ses activités. 
Finalement, ajoutons que dans le cas d'Imagem, comme dans bien d'autres cas d'entreprise, les intervenants gouvernementaux n'ont pu dégager les ressources humaines de soutien nécessaires à une gestion équilibrée. Pourtant notre expérience de diagnostic organisationnel dans l'entreprise prouve bien la capacité rapide de prise en mains des entrepreneurs, lorsqu'ils sont adéquatement appuyés dans leur recherche de solutions et dans les nombreuses étapes de 1'implantation. L'ère du nouveau consultant est peut-être arrivée!

Pour terminer, nous osons croire que notre mémoire contribuera même de façon modeste, à susciter une réflexion sur la démarche de diagnostic-intervention adoptée, pour opérer un changement positif dans l'entreprise Imagem et sur l'efficacité d'une telle méthode pour améliorer notre compréhension du monde des petites et moyennes organisations. 


\section{B I B L I OG RA P H I E}

1- BAMBeRgER, Ingolf, "Le management stratégique dans les PME", Revue Direction et gestion des entreprises, $21 \mathrm{e}$ année, juiliet et août 1985, p. 15 à 26

2- BERGERON, J.L. et al, Les aspects humains de l'organisation, Gaetan Morin Editeur, Chicoutimi, 1979, 337 pages

3- BERNoUX, Philippe, La sociologie des organisations, Editions du seuil, extraits, pages 118 à 156

4- BRUNET, Luc, Le climat de travail dans les organisations, Les Editions Agence d'Arc Inc, Ottawa, 1983, 141 pages

5- CHICHA, Joseph, Le management stratégique dans l'entreprise et dans la pME, fascicule 1, université du Québec à Trois-Rivières, 1986,122 pages

6- COLLERETTE, P et DELISLE, G, Le changement planifié, Les Editions Agence d'Arc Inc, Ottawa, 1982, 213 pages

7- CONSEIL RÉgional DE DÉVELOPPEMENT DE L'OUTAOUAis et al, Notre région en chiffres, 1984,124 pages

8- Cote, Laval, session de formation pour conseillers et conseillères en démarrage d'entreprise, Ministère de I'Industrie et du Commerce, Gouvernement du Québec, 1987 , 71 pages

9- D'AMBOISE, G. et GASSE, Y., La PME manufacturière, 12 cas québécois, Gaetan Morin Editeur, 1984, Chicoutimi, 198 pages

10- DELL'ANIELLO, paul et PERREAULT, Yvon Comment la pME peut survivre aux années 80, Editions G. Vermette, 1985, ottawa, 245 pages

11- DuCASSE, Christiane, "Télévision, début de saison", qui fait quoi, septembre/octobre $1987, \mathrm{n}^{\circ} 42$, page 7

12- ETHIER, C. et DUCASSE, C. "Dans le cirque de la production", Qui fait quoi, août/septembre $1987, \mathrm{n}^{\circ} 41$, pages 13 et 14

13- GAGNON, Jean H., La PME face à la crise financière, Les Editions Agence d'ArC Inc, Ottawa, 1983, 180 pages 
14- GASSE, Yvon et al, "L'entrepreneurship: une stratégie de recherche et d'intervention pour le développement", Revue PMO, Vol $1, \mathrm{n}^{\circ} 5,1985, \mathrm{p}, 8$ à 22

15- GÉLINIER, Octave, Stratégie de l'entreprise et motivation des hommes, Editions Hommes et Techniques, Paris, 1984, 311 pages

16- HURTUBISE, Rolland, L'administrateur québécois et les systèmes, Les Editions Agence d'Arc Inc, Ottawa, 1980, 140 pages

17- LANDRY, Maurice, Doit-on concevoir ou analyser les problèmes complexes, Université Laval, 1980, 31 pages

18- LEFRANCOIS, Pierre "Systèmes et phénomènes complexes", Revue PMO, Vo $11, \mathrm{n}^{\circ} 2,1983, \mathrm{p}, 20$ et 21

19- LORRAIN, J et DUSSAULT, L., "Les entrepreneurs en phase de démarrage: profil psychologique et comportement de gestion, Revue PMO, Vol 2, $\mathrm{n}^{\circ} 1,1986$, p. 26 à 36

20- MARTINET, Alain C., Management stratégigue: organisation et politique, extraits, McGraw-Hill, Paris, 1984, p. 45 à 118

21- MARTINet, Alain C., Stratégie, Librairie Vuibert, Paris, 1983,322 pages

22- MINTZBERG, Henri, Le pouvoir dans les organisations, Les Editions d'organisation, 1986, Paris, 679 pages

23- NACIRI, Ahmed, "Petites entreprises, le financement limité et difficile", Revue PMO, Vol 1, $\mathrm{n}^{\circ} 5,1985, \mathrm{p}$. 30 a 31

24- PARENT, Réjean, "Les causes des faillites des entreprises", Revue PMO, Vol $1, \mathrm{n}^{\circ} 2,1984, \mathrm{p}, 15$

25- PAyetTe, Michel, "La production non destinée aux salles, L'incola de 1'industrie du cinéma", qui fait quoi, $\mathrm{mai} / \mathrm{avril} 1987, \mathrm{n}^{\circ} 36, \mathrm{p}, 8$ à 12

26- PETERSON, Rein, petites et moyennes entreprises, pour une économie équilibrée, Le Cercle du Livre de France Ltée, Ottawa, 1978, 249 pages

27- PRÉVOST, Paul, Le diagnostic - intervention: une approche systémique au diagnostic organisationnel et à la recherche-action, LEER, Chicoutimi, 1983, 92 pages 
28- RIGNY, André J., Diagnostic organisationnel, cas vécus, Les Editions Agence d'ArC Inc, Ottawa, 1982, 365 pages

29- ROBIDOUX, Jean, Les crises administratives dans les pME en croissance, Gaetan Morin \& associée Ltée, chicoutimi, 1978,131 pages

30- ROY, PRÉVOST, BELLEY et al, "Vers une définition du concept de la recherche-action", Revue PMO, Vol $1, \mathrm{n}^{\circ} 1$, $1982, p \cdot 20$ à 23

31- ROY, Rita, "Les problèmes des PMO", Revue PMO, Vol 1 , $n^{\circ}$ $2,1983, \mathrm{p} .7$ à 10

32- Tremblay, Mario, "Guide de planification pour PME", Revue PMO, Vol $1, \mathrm{n}^{\circ} 2,1983, \mathrm{p}, 12$ à 19

33- VALLERAND et al, Les PME au Québec, etat de la situation, Rapport du ministre délégué aux PME, Gouvernement du Québec, Québec, 1987,320 pages

34- WALLOT, Hubert, "Les aspects humains de la crise dans la petite entreprise", Revue PMO, Vol $1, \mathrm{n}^{\circ} 2,1984, \mathrm{p}, 12$ à 14

35- WRIGHTSMAN, SELLTiz, COOK, Les méthodes de recherche en sciences sociales, Les Editions HRW Ltée, Anjou, 1977, 606 pages 


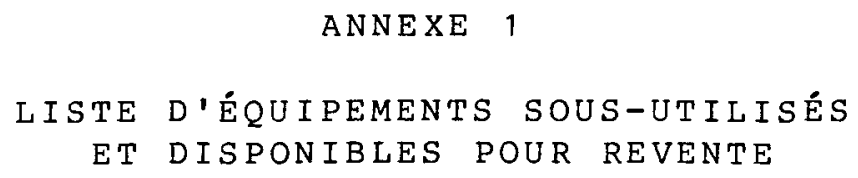

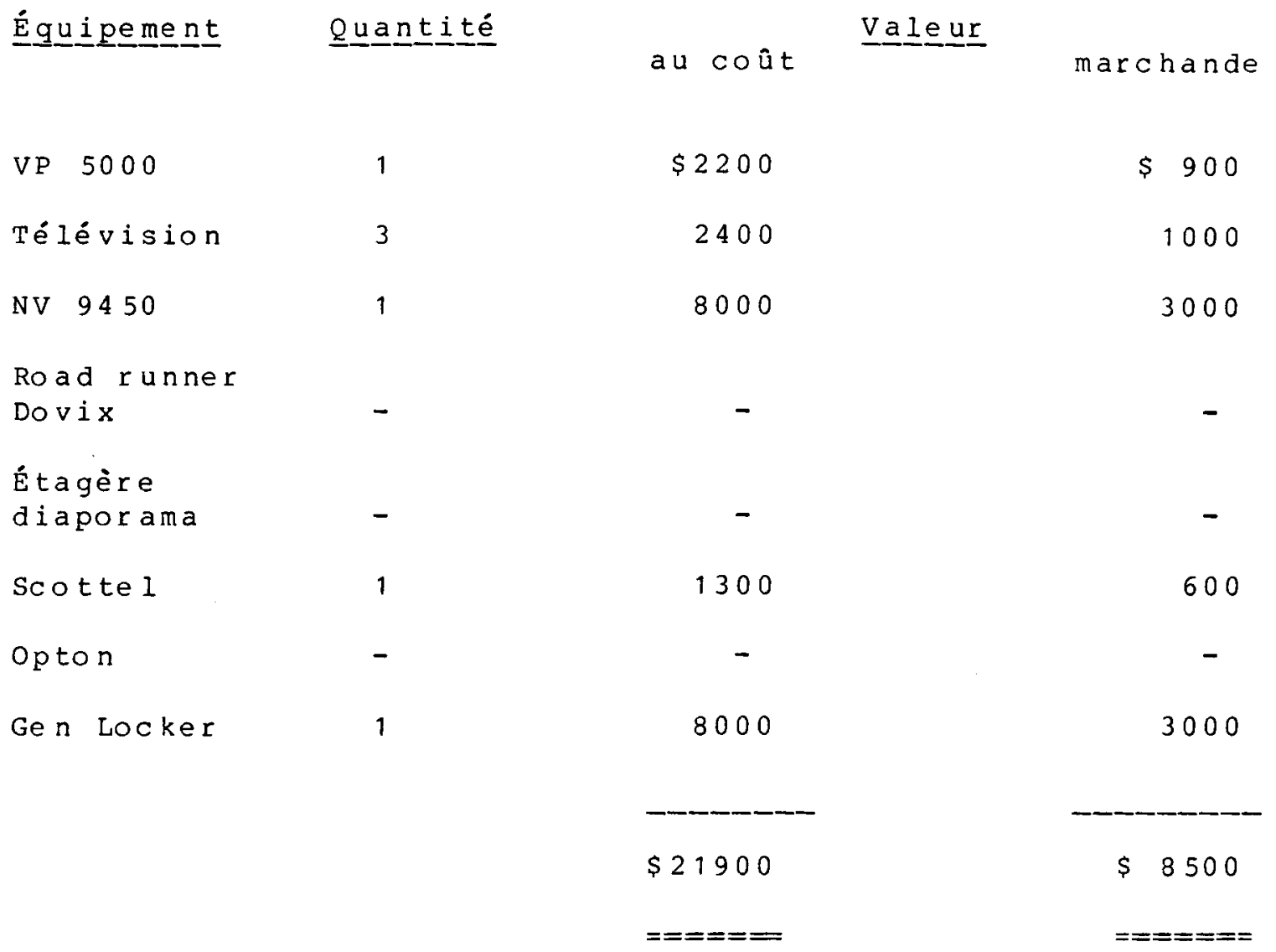




\begin{tabular}{|c|c|c|c|}
\hline \multicolumn{4}{|c|}{ ANNEXE 2} \\
\hline \multicolumn{4}{|c|}{ IMAGEM } \\
\hline \multicolumn{4}{|c|}{ BILANS COMPARATIFS } \\
\hline & $\begin{array}{l}29 \mathrm{fé} v \\
1987 \\
(8 \text { mois) }\end{array}$ & $\begin{array}{l}30 \text { juin } \\
1987 \\
(12 \text { mois })\end{array}$ & $\begin{array}{l}31 \text { oc tobre } \\
1987 \\
(4 \text { mois })\end{array}$ \\
\hline \multicolumn{4}{|l|}{ ACT I F } \\
\hline Encaisse & $(13)$ & $(1294)$ & - \\
\hline \multicolumn{4}{|l|}{ Encaisse des immobilisa- } \\
\hline tions & 225 & $(3881)$ & 1112 \\
\hline Débi te urs & 8145 & 28661 & 27469 \\
\hline Inventaire & 1382 & 1382 & 1780 \\
\hline \multirow[t]{2}{*}{ Frais payés d'avance } & 1556 & 2500 & - \\
\hline & 11295 & 27368 & 30361 \\
\hline \multirow{3}{*}{$\begin{array}{l}\text { Equipements prof. } \\
\text { Bureau et informatique }\end{array}$} & 90162 & 90322 & 86106 \\
\hline & 19988 & 19988 & 12801 \\
\hline & 110150 & 110310 & 98907 \\
\hline \multirow[t]{4}{*}{$\begin{array}{l}\text { Amortissement } \\
\text { accumulé }\end{array}$} & $(40081)$ & $(40081)$ & $(34094)$ \\
\hline & 70069 & 70229 & 64813 \\
\hline & $\$ 81364$ & $\$ 97597$ & $\$ 95174$ \\
\hline & $=====$ & $=====0$ & $=======$ \\
\hline \multicolumn{4}{|l|}{ PASSIF } \\
\hline \multirow{4}{*}{$\begin{array}{l}\text { Comptes fourn. } \\
\text { Créditeurs/ frais courus } \\
\text { Emprunt à court terme }\end{array}$} & 18156 & 24520 & 21849 \\
\hline & $5 \quad 11883$ & 8437 & 3783 \\
\hline & 6453 & 6453 & 5500 \\
\hline & 36492 & 39410 & 31132 \\
\hline \multirow{3}{*}{$\begin{array}{l}\text { Emprunt à long terme } \\
\text { Avances }\end{array}$} & 51825 & 47425 & 44125 \\
\hline & 23 & 53 & 53 \\
\hline & 88340 & 86888 & 75310 \\
\hline \multicolumn{4}{|l|}{ AVOIR DES ACTIONNAIRES } \\
\hline \multirow{4}{*}{$\begin{array}{l}\text { Capital actions } \\
\text { Subventions PECEC } \\
\text { Bénéfices (pertes) } \\
\text { non répartis }\end{array}$} & 20489 & 23489 & 23489 \\
\hline & 63000 & 70000 & 70000 \\
\hline & $(90465)$ & $(82780)$ & $(73625)$ \\
\hline & $\begin{array}{l}\$ 81364 \\
=====\end{array}$ & $\begin{array}{l}\$ 97597 \\
=====\end{array}$ & $\begin{array}{l}\$ 95174 \\
=====\end{array}$ \\
\hline
\end{tabular}




\begin{tabular}{|c|c|c|c|}
\hline & ANNEXE 3 & & \\
\hline & IMAGEM & & \\
\hline RÉSULTATS & D'EXPLOITATION & COMPARATIFS & \\
\hline & $\begin{array}{l}29 \text { fév. } \\
1987\end{array}$ & $\begin{array}{c}30 \text { juin } \\
1987\end{array}$ & $\begin{array}{r}31 \text { oc tobre } \\
1987\end{array}$ \\
\hline & ( 8 mois) & ( 12 mois) & ( 4 mois) \\
\hline VENTES & & & \\
\hline Diffusion opton & $\$ \quad 4159$ & $\$ 5639$ & $\$$ \\
\hline Productions audiovi- & & & \\
\hline sue 1 les & 25872 & 46209 & 6523 \\
\hline Ho noraires & 47550 & 82219 & 45714 \\
\hline Escomptes & $(2249)$ & $(2392)$ & 216 \\
\hline Services & 6875 & 8655 & 1576 \\
\hline Autres & - & - & 492 \\
\hline & 82207 & 140330 & 54521 \\
\hline COUTTS DE PRODUCTION & & & \\
\hline Matières premières & 3339 & 7231 & 1784 \\
\hline Main-d'oeuvre & 10174 & 10594 & 2757 \\
\hline Bé néfices marginaux & - & - & 86 \\
\hline Loc ation & 5464 & 10288 & 5574 \\
\hline Sous-traitance & 21870 & 32547 & 13472 \\
\hline Accessoires & 313 & 422 & 68 \\
\hline Transport & 29 & 386 & 1378 \\
\hline Amortissement & 11762 & 11762 & 3921 \\
\hline & 52951 & 73230 & 29040 \\
\hline BÉNÉFICES BRUTS & 29256 & 67100 & 25481 \\
\hline FRAIS GÉNÉRAUX & & & \\
\hline D'EXPLOITATION & 54416 & 78213 & 26461 \\
\hline PERTE NETTE & $\begin{array}{l}\$ 25160 \\
====\end{array}$ & $\begin{array}{l}\$ 11113 \\
====\end{array}$ & $\begin{array}{l}\$ 980 \\
=====\end{array}$ \\
\hline
\end{tabular}




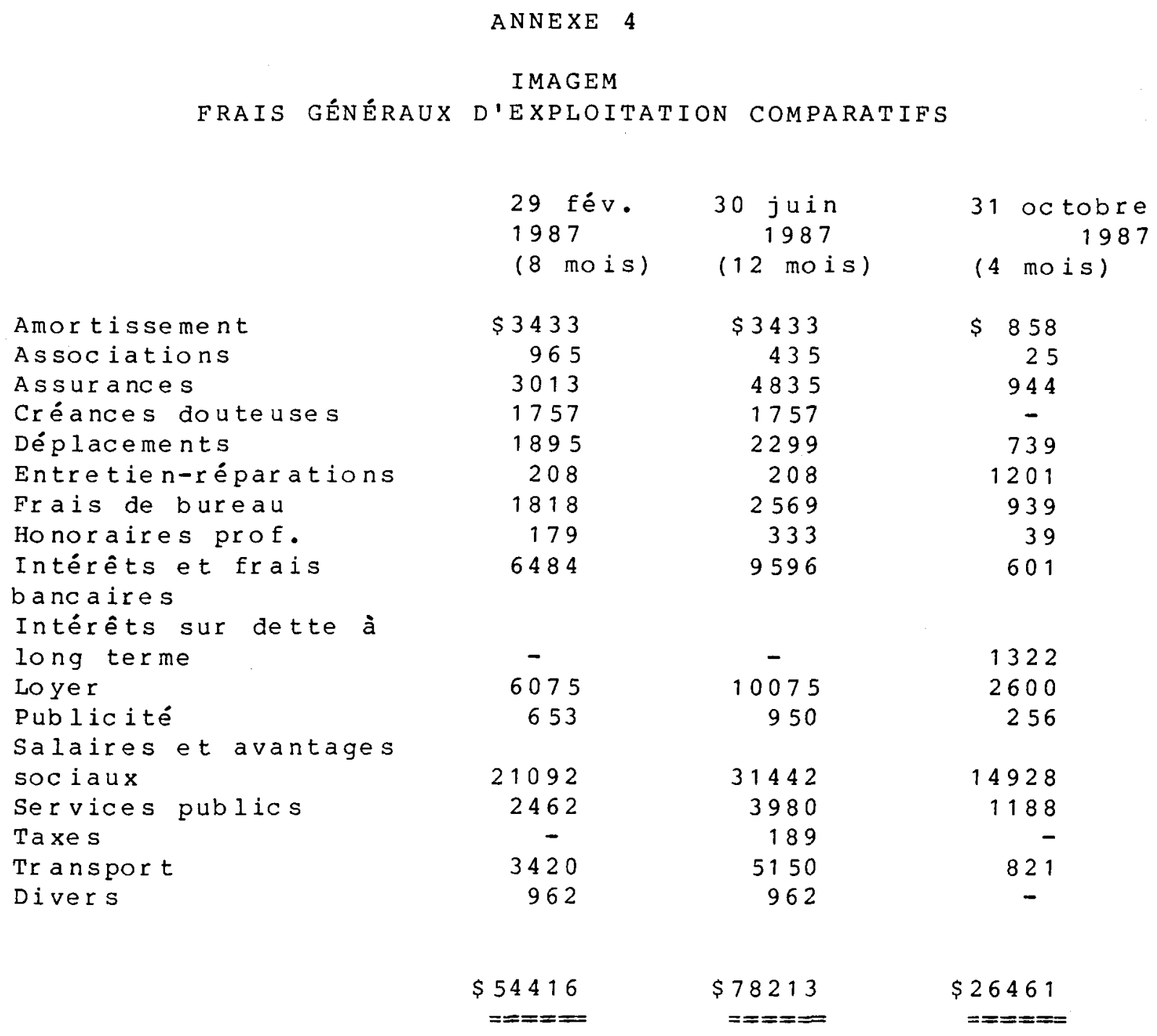




\section{PARAMÉTRES UTILISÉS DANS LE CALCUL DU PRIX DE REVIENT}

1) LES COUTTS FIXES MENSUELS

Au 30 juin 87 Au 31 octobre 87

Associations

Assurances

Créances douteuses

Déplacements

Entretien-réparations

Frais de bureau

Honoraires professionnels

Intérêts et frais bancaires

Intérêts à long terme

Loyer

Publicité

Salaires et avantages sociaux 2620

Services publics

Taxes

Transport

Diver $\mathrm{s}$

Amortissement $B$

Amortissement $E$

36

403

146

192

17

214

28

800

$-$

840

79

332

16

429

80

286

980

$\$ 7498$

$===$
6

236

$1 \overline{85}$

300

235

10

150

331

650

64

3732

297

$-$

205

$-$

286

980

$\$ 7596$

$===$

Donc on peut affirmer que les frais fixes mensuels sont d'environ $\$ 8000$, pour être conservateur. 
2) LA CAPACITÉ DE PRODUCTION HEBDOMADAIRE

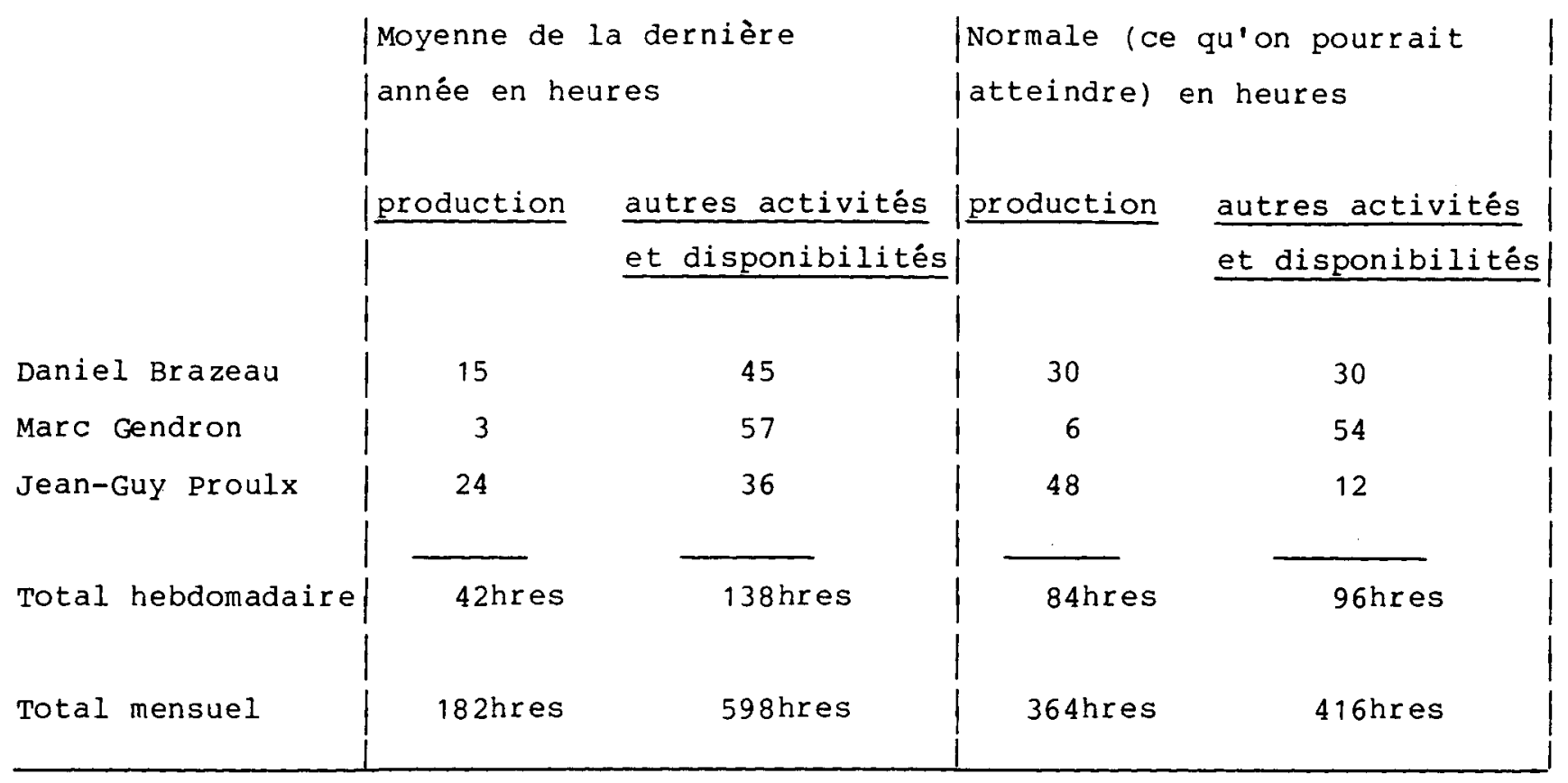

Ainsi, les activités de production de l'entreprise pourraient doubler au cours de la prochaine année sans occasionner de difficultés majeures dans les opérations.

3) LE TAUX HORAIRE DE FACTURATION DES FRAIS FIXES (les frais variables étant facturés de façon distincte)

coûts fixes mensuels

capacité de production moyenne mensuelle
$=\$ 8000=$

182 hres

$=\$ 44$ par heure 
ANNEXE 6

CALCUL DU PRIX DE REVIENT

\begin{tabular}{|c|c|c|c|c|c|c|c|c|}
\hline \multirow[t]{2}{*}{ Nom du produit } & \multirow[t]{2}{*}{ Date } & \multirow[t]{2}{*}{ Catégorie } & \multirow[t]{2}{*}{$\begin{array}{l}\text { Prix de } \\
\text { vente }\end{array}$} & \multicolumn{5}{|c|}{$\begin{array}{l}\text { Prix de revient sur base de cap. moy } \\
\text { Taux } H=\frac{\text { coûts fixes mensuels }}{182 \text { hres de prod. men }} \\
\begin{array}{l|l|l}\text { Frais } & \text { Frais } & \text { Profit } \\
\text { fixes } & \text { variables } & \text { net } \\
\end{array}\end{array}$} \\
\hline & & & & hres & $\$$ & $\$$ & $\$$ & 8 \\
\hline $\begin{array}{l}\text { FÉDÉRATION DES } \\
\text { CAISSES POP } \\
\text { ONTARIO }\end{array}$ & $03-87$ & DIA PORAMA & $7452 \$$ & 175 & 7700 & 1600 & $(1752)$ & -23 \\
\hline VOYAGE EL AIR & $09-87$ & DIAPORAMA & 2400 & 38 & 1672 & 1450 & $(722)$ & -30 \\
\hline $\begin{array}{l}\text { DIRECTION } \\
\text { JEUNESSE }\end{array}$ & $10-87$ & DIAPORAMA & 6373 & 61 & 2684 & 3688 & 1 & 0 \\
\hline $\begin{array}{l}\text { SOCIÉTÉ } \\
\text { D'AMÉNA GEMENT } \\
\text { DE L 'OUTAOUAIS }\end{array}$ & $03-87$ & DIAPORAMA & 4763 & 90 & 3960 & 1400 & $(597)$ & -13 \\
\hline RIFAS & $05-87$ & VIDÉO & 15000 & 132 & 5808 & 8500 & 692 & 5 \\
\hline $\begin{array}{l}\text { VILLE DE } \\
\text { GATINEAU }\end{array}$ & $08-87$ & VIDÉO & 20800 & 250 & 11000 & 9450 & 350 & 2 \\
\hline $\begin{array}{l}\text { ASS. DES } \\
\text { ENSEIGNANTS } \\
\text { FRANCO-ONT }\end{array}$ & $05-86$ & VIDÉO & 15326 & 190 & 8360 & 7100 & $(134)$ & -1 \\
\hline $\begin{array}{l}\text { STATION } \\
\text { CKCH }\end{array}$ & $08-87$ & VIDÉO & 3750 & 76 & 3344 & 3390 & $(2984)$ & -80 \\
\hline $\begin{array}{l}\text { PLACE DU } \\
\text { MARCHÉ }\end{array}$ & $08-86$ & VIDÉO & 3500 & 12 & 528 & 2225 & 747 & 21 \\
\hline $\begin{array}{l}\text { PRODUCTIONS } \\
24-30 \\
\text { LAC MEECH }\end{array}$ & $09-87$ & VIDÉO & 9110 & 126 & 5544 & 4014 & $(448)$ & -5 \\
\hline $\begin{array}{l}\text { PRODUCT IONS } \\
24-30 \\
\text { DOMAINE } \\
\text { MAC KENZ IE K ING }\end{array}$ & 09-87 & VIDÉO & 9100 & 100 & 4400 & 4000 & 700 & 8 \\
\hline $\begin{array}{l}\text { PRODUCTIONS } \\
24-30 \\
\text { PISTES } \\
\text { CYCLABLES }\end{array}$ & $10-87$ & VIDÉO & 9100 & 108 & 4752 & 3970 & 378 & 4 \\
\hline $\begin{array}{l}\text { PRODUCTIONS } \\
24-30 \\
\text { LAC DES FÉES }\end{array}$ & $10-87$ & VIDÉO & 9100 & 88 & 3872 & 4570 & 658 & 7 \\
\hline
\end{tabular}


ANNEXE 6 (suite)

\begin{tabular}{|c|c|c|c|c|c|c|c|c|}
\hline \multirow[t]{2}{*}{ Nom du produit } & \multirow[t]{2}{*}{ Date } & \multirow[t]{2}{*}{ Catégorie } & \multirow[t]{2}{*}{$\mid \begin{array}{l}\text { Prix de } \\
\text { vente }\end{array}$} & \multicolumn{5}{|c|}{$\begin{array}{l}\text { Prix de revient sur base de cap. moy } \\
\text { Taux } H=\frac{\text { coûts fixes mensuels }}{182 \text { hres de prod. men. }} \\
\text { Frais } \\
\begin{array}{l|l|l}\text { fixes } & \begin{array}{l}\text { Frais } \\
\text { variables }\end{array} & \begin{array}{l}\text { Profit } \\
\text { net }\end{array} \\
\end{array}\end{array}$} \\
\hline & & & & hres & $\$$ & $\$$ & $\$$ & 8 \\
\hline $\begin{array}{l}\text { VILLE DE } \\
\text { GATINEAU }\end{array}$ & $05-87$ & $\begin{array}{l}\text { SERVICES } \\
\text { TECHN. }\end{array}$ & 600 & 8 & 352 & 210 & 38 & 6 \\
\hline VILLE DE & & & & & & & & \\
\hline BUCKINGHAM & $10-87$ & KIOSQUE & 840 & 15 & 660 & 389 & $(209)$ & -25 \\
\hline CRSSO & $10-86$ & KIOSQUE & 4939 & 83 & 3652 & 1463 & 176 & -4 \\
\hline POLE LITE & $09-87$ & PHOTOS & 700 & 16 & 704 & 310 & $(314)$ & -45 \\
\hline GENDRON & & & & & & & & \\
\hline LEFEBVRE & $10-87$ & PHOTOS & 670 & 22 & 968 & 440 & $(738)$ & -110 \\
\hline CAISSE POP & & & & & & & & \\
\hline YOUVILLE & $11-87$ & PHOTOS & 125 & 2 & 88 & 25 & 12 & 10 \\
\hline POLE LITE & $02-87$ & PHOTOS & 1618 & 9 & 396 & 855 & 367 & 23 \\
\hline TOTAL & & & $125356 \$$ & & 70436 & $59049 \$$ & $(4129)$ & $-3 \frac{8}{6}$ \\
\hline
\end{tabular}


ANkEXE 7 Budget de caisse previsionnel (an 1 - Imagen

\begin{tabular}{|c|c|c|c|c|c|c|c|c|c|c|c|c|c|}
\hline \multirow[t]{2}{*}{ Du: ler nov 87} & \multicolumn{2}{|c|}{ au: 31 oct 88} & \multirow[b]{2}{*}{ DEC } & \multirow[b]{2}{*}{ JAN } & \multirow[b]{2}{*}{ PEV } & \multirow[b]{2}{*}{ MABS } & \multirow[b]{2}{*}{ AVRIL } & \multirow[b]{2}{*}{ MAI } & \multirow[b]{2}{*}{ JUIN } & \multirow[b]{2}{*}{ JUILIET } & \multirow[b]{2}{*}{ AOUT } & \multirow[b]{2}{*}{ SEPT } & \multirow[b]{2}{*}{ OCT } \\
\hline & Total & HOV & & & & & & & & & & & \\
\hline Bacaisse debut 1 & $(\$ 4,388)$ & $(\$ 4,388)$ & $(\$ 3,133)$ & $\$ 622$ & $\$ 1,628$ & $\$ 8,922$ & $\$ 6,960$ & $\$ 11,477$ & $\$ 17,457$ & $\$ 13,924$ & $\$ 10,558$ & $\$ 7,468$ & $\$ 9,05$ \\
\hline \multicolumn{14}{|l|}{ Recettes } \\
\hline Ventes encaissees & $\$ 241,868$ & $\$ 18,675$ & $\$ 20,508$ & $\$ 15,711$ & $\$ 37,300$ & $\$ 21,724$ & $\$ 28,225$ & $\$ 23,725$ & $\$ 18,000$ & $\$ 10,000$ & $\$ 10,000$ & $\$ 18,000$ & $\$ 20,00$ \\
\hline $\begin{array}{l}\text { Boprunt a c/t } \\
\text { Autres }\end{array}$ & $\begin{array}{r}\$ 0 \\
\$ 5,075\end{array}$ & $\begin{array}{l}\text { \$0 } \\
\text { \$0 }\end{array}$ & & & & & & & & & & & \\
\hline Mise de fonds & $\$ 0$ & & & & & & & & & & & & \\
\hline Sous- & $\$ 3,570$ & \$0 & $\$ 0$ & $\$ 357$ & $\$ 357$ & $\$ 357$ & $\$ 357$ & $\$ 357$ & $\$ 357$ & $\$ 357$ & $\$ 357$ & $\$ 357$ & \\
\hline Total & $\$ 250,513$ & $\$ 18,675$ & $\$ 20,508$ & $\$ 21.14\}$ & $\$ 37,657$ & $\$ 22,081$ & $\$ 28,582$ & $\$ 24,082$ & $\$ 18,357$ & $\$ 10,357$ & $\$ 10,357$ & $\$ 18,357$ & $\$ 20,357$ \\
\hline \multicolumn{14}{|l|}{ ebourses } \\
\hline DAS courant & 3,187 & $\$ 856$ & $\$ 630$ & $\$ 568$ & $\$ 1,142$ & $\$ 1,14$ & $\$ 1,427$ & $\$ 1,1$ & $\$ 1,142$ & $\$ 1,427$ & $\$ 1,142$ & $\$ 1,142$ & $\$ 1,427$ \\
\hline Ach & 29 & $\$ 195$ & s & $\$ 7$ & $\$ 1$, & & & & & & & & $\$ 600$ \\
\hline Sala & $\$ 37,542$ & $\$ 2,552$ & $\$ 2,115$ & $\$ 3,058$ & $\$ 3,058$ & $\$ 3,823$ & $\$ 3,058$ & $\$ 3$, & $\$ 3$, & $\$ 3,058$ & $\$ 3,058$ & $\$ 3,823$ & $\$ 3,058$ \\
\hline Sous & $\$ 89,913$ & \$0 & $\$ 7,500$ & $\$ 5,101$ & $\$ 15,666$ & $\$ 8,652$ & $\$ 11,382$ & $\$ 9,492$ & $\$ 7$. & $\$ 4$ & $\$ 4$, & $\$ 7,560$ & $\$ 8,600$ \\
\hline gouv. & $\$ 4,096$ & $\$ 350$ & $\$ 255$ & $\$ 215$ & $\$ 336$ & $\$ 336$ & $\$ 420$ & $\$ 336$ & $\$ 336$ & $\$ 420$ & $\$ 336$ & $\$ 336$ & $\$ 420$ \\
\hline c., chauf. & $\$ 11,800$ & $\$ 600$ & \$0 & $\$ 2,200$ & $\$ 1,000$ & $\$ 1,000$ & $\$ 1,000$ & $\$ 1,000$ & $\$ 1,000$ & $\$ 1,000$ & $\$ 1,000$ & $\$ 1,000$ & $\$ 1,000$ \\
\hline Taxes & $\$ 575$ & $\$ 0$ & \$0 & $\$ 0$ & $\$ 525$ & $\$ 50$ & $\$ 0$ & $\$ 0$ & $\$ 0$ & $\$ 0$ & \$o & \$o & \$o \\
\hline feu/vie & $\$ 3,32\}$ & $\$ 683$ & $\$ 715$ & $\$ 715$ & $\$ 90$ & $\$ 90$ & $\$ 90$ & $\$ 90$ & $\$ 90$ & $\$ 90$ & $\$ 90$ & $\$ 90$ & $\$ 490$ \\
\hline Paieme & $\$ 3,685$ & $\$ 0$ & $\$$ & $\$ 670$ & $\$ 335$ & $\$ 335$ & $\$ 335$ & $\$ 335$ & $\$ 335$ & 5 & $\$ 335$ & $\$ 335$ & $\$ 335$ \\
\hline Loc. 1 & $\$ 1,800$ & $\$ 150$ & $\$ 150$ & $\$ 1$ & & $\$ 150$ & $\$ 1$ & & $\$$ & 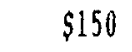 & $\$ 150$ & 0 & $\$ 150$ \\
\hline esent & $\$ 1,800$ & $\$ 150$ & \$0 & $\$ 3$ & $\$ 1$ & $\$ 150$ & $\$ 150$ & 0 & $\$ 1$ & $\$$ & $\$ 150$ & $\$ 150$ & $\$ 150$ \\
\hline Depe & $\$ 1,755$ & $\$ 135$ & $\$ 135$ & $\$ 1$ & $\$ 1$ & $\$ 150$ & $\$ 150$ & $\$ 150$ & $\$ 1$ & $\$ 1$ & $\$ 150$ & $\$ 150$ & $\$ 150$ \\
\hline Tele & $\$ 4,188$ & $\$ 349$ & $\$ 349$ & $\$ 3$ & $\$ 3$ & $\$ 3$ & $\$ 3$ & $\$ 3$ & $\$ 3$ & $\$ 3$ & $\$ 349$ & $\$ 349$ & $\$ 34$ \\
\hline Pour & $\$ 2,400$ & $\$ 200$ & $\$ 0$ & $\$ 400$ & $\$ 2$ & $\$ 200$ & $\$ 200$ & $\$ 200$ & $\$ 2$ & $\$ 200$ & $\$ 200$ & $\$ 200$ & $\$ 20$ \\
\hline Honol & $\$ 3,200$ & $\$ 500$ & \$0 & $\$ 1,000$ & $\$ 100$ & $\$ 700$ & $\$ 600$ & $\$ 0$ & $\$ 1$ & \$o & $\$ 100$ & \$0 & $\$ 10$ \\
\hline unt $c / t$ & $\$ 750$ & $\$ 100$ & $\$ 100$ & $\$ 100$ & $\$ 50$ & $\$ 50$ & $\$ 50$ & $\$ 50$ & $\$ 50$ & $\$ 50$ & $\$ 50$ & $\$ 50$ & 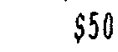 \\
\hline Autres & $\$ 11,887$ & $\$ 4,703$ & & & & $\$ 312$ & & & $\$ 5,000$ & $\$ 936$ & $\$ 936$ & & \\
\hline Int. em & $\$ 2,999$ & $\$ 410$ & $\$ 404$ & $\$ 250$ & $\$ 24\}$ & $\$ 236$ & $\$ 229$ & $\$ 222$ & $\$ 215$ & $\$ 208$ & $\$ 201$ & $\$ 194$ & $\$ 18$ \\
\hline Cap. e & $\$ 9,200$ & $\$ 1,100$ & $\$ 1,100$ & $\$ 700$ & $\$ 700$ & $\$ 700$ & $\$ 700$ & $\$ 700$ & $\$ 700$ & $\$ 700$ & $\$ 700$ & $\$ 700$ & $\$ 70$ \\
\hline yables & $\$ 21,849$ & $\{3,387$ & $\$ 3,000$ & $\$ 2,500$ & $\$ 5,000$ & $\$ 5,000$ & $\$ 2,962$ & \$0 & So & $\$ 0$ & 50 & $\$ 0$ & . \\
\hline & & $\$ 1,000$ & $\$ 300$ & $\$ 1,000$ & so & so & so & $\$ 0$ & \$0 & \$0 & \$0 & $\$ 0$ & \\
\hline Total(debourses) 3 & $\$ 234,678$ & $\$ 17,420$ & $\$ 16,753$ & $\$ 20,137$ & $\$ 30,363$ & $\$ 24,043$ & $\$ 24,665$ & $\$ 18,102$ & $\$ 21,890$ & $\$ 13,723$ & $\$ 13,447$ & $\$ 16,769$ & $\$ 17,96$ \\
\hline caisge a la & 1,447 & $(\{3,133)$ & 622 & .628 & $\$ 8,922$ & $\$ 6,960$ & $\$ 11,477$ & $\$ 17,457$ & $\$ 13,924$ & $\$ 10,558$ & $\$ 7,468$ & $\$ 9,056$ & 44 \\
\hline
\end{tabular}


ANABXE 8 Budget de caisse previsionnel (an 2) - Inagem

Du: ler nov 88

Total au: 31 oct 89

Rncaisse debut

$\$ 11,447$

11,447

$14 \quad 15$

16

17

18

19

20

21

22

23

24

Recettes

Ventes encaissees

$\$ 245,000$

Buprunt a c/t

Autres

Nise de fonds

Sous-location

Total (recettes) 2

50

$\$ 249,500$

$\$ 375$

$\$ 375$

$\$ 375$

$\$ 375$

$\$ 23,375 \quad \$ 23,375$

$\$ 23,375$

$\$ 18,375$

$\$ 13,375$

$\$ 13,375$

$\$ 24,375$

$\$ 373$

Debourses

Achats (38)

Salaires nets

$\$ 15,584$

$\$ 1,199$

$\$ 1,199$

\section{$\$ 1,498$}

$\$ 1,199$

$\$ 1,199$

$\$ 1,498$

$\$ 1,199$

$\$ 41,744 \quad \$ 3,211$ $\$ 102,900$

Sous-contrats

$\$ 4,588$

$\$ 9,240$

$\$ 4,014$

$\$ \hat{3}, 211$

$\$ 3,211$

$\$ 690$

$\$ 690$

$\$ 690$

$\$ 1,199$

$\$ 1,498$

$\$ 1,199$

$\$ 9,660 \quad \$ 9,660 \quad \$ 9,660$

$\$ 4,014$

$\$ 3,211$

Loyer, elec, chauf

Loc. II equip

Taxes et permis

Ass. feu/vie

Pajement roulant

Prais de represent

Depenses de roul.

Telephone

Pournitures de bur

$\$ 12,600$

$\$ 353$

$\$ 9,240$

\$7, 560

$\$ 8,820$

$\$ 353$

$\$ 960$

$\$ 9,660$

$\$, 560$
$\$ 357$

$\$ 5,460$

$\$ 390$

$\$ 1,199 \quad \$ 1,498$

$\$ 1,050$

$\$ 1,050$

$\$ 1.050$

$\$ 1,050$

$\$ 1,050$

$\$ 1,050$

$\$ 1,050$

$\$ 1,050$

$\$ 601$

50
5490

$\$ 0$

$\$ 150$

$\$ 150$

$\$ 150$

$\$ 150$

$\$ 150$

$\$ 1,050$

$\$ 2,280$

$\$ 490$

$\$ 490$

$\$ 490$

$\$ 551$

$\$ 50$
$\$ 90$

$\$ 0$
$\$ 90$

$\$ 0$
590

$\$ 0$
$\$ 90$

$\$ 0$
$\$ 1,896$

$\$ 158$

$\$ 0$
$\$ 158$

$\$ 0$
$\$ 158$

$\$ 0$
$\$ 158$

$\$ 4,416$

$\$ 4,392$

$\$ 368$

$\$ 368$

$\$ 368$

Honoraires prof.

$\$ 2,520$

$\$ 2,000$

$\$ 210$

$\$ 366$

$\$ 366$

$\$ 368$

$\$ 0$
$\$ 158$

$\$ 0$
$\$ 158$

$\$ 0$

$\$ 0$
$\$ 158$

$\$ 0$
$\$ 158$

$$
\$ 368
$$

$\$ 368$

$\$ 366$

$\$ 368$

$\$ 366$

Int, emprunt $\mathrm{c} / \mathrm{t}$

$\$ 636$

$\$ 2,275$

Autres avan.soc

Int. emprunt $1 / t$

$\$ 1,698$

$\$ 8,400$

$\$ 0$
50

$\$ 0$
$\$ 53$

\$0

$\$ 210$

$\$ 210$

$\$ 210$

$\$ 210$

$\$ 210$

$\$ 368$

$\$ 366$

$\$ 210$
50

$\$ 0$
$\$ 53$

$\$ 180$
$\$ 700$

\$173

$\begin{array}{rr}\$ 53 & \$ 53 \\ & \$ 328\end{array}$

$\$ 53$

$\$ 53$

\$131

$$
\begin{aligned}
& \$ 145 \\
& \$ 700
\end{aligned}
$$$$
\$ 138
$$

$\$ 131$
$\$ 700$

50
50

$\$ 0$

50
50

$\$ 0$
$\$ 0$

$\$ 0$

50
50

$\$ 0$
$\$ 0$

50
50

Total(debourses) 3

$\begin{array}{lllllll}\$ 43,267 & \$ 15,434 & \$ 18,625 & \$ 19,839 & \$ 23,146 & \$ 26,300 & \$ 30,255\end{array}$

Racaisse a la fi

11 plus 2 goins 31

$\$ 18,396 \quad \$ 16,942 \quad \$ 15,442$

$\$ 0$
$\$ 90$
$\$ 0$
$\$ 158$
$\$ 368$
$\$ 366$
$\$ 210$
$\$ 200$
$\$ 53$
$\$ 973$
$\$ 124$
$\$ 700$
$\$ 0$
$\$ 0$

$, 460 \$ 10,080 \quad \$ 10,500$

$\$ 353 \quad \$ 353 \quad \$ 441$

$\begin{array}{rrr}\$ 1,050 & \$ 1,050 & \$ 1,050 \\ \$ 150 & \$ 150 & \$ 150\end{array}$

\$0 $50 \quad \$ 0$

$\$ 90 \quad \$ 90 \quad \$ 90$

$$
\begin{array}{rrr}
\$ 0 & \$ 0 & \$ 0 \\
\$ 158 & \$ 58 & \$ 158
\end{array}
$$

$\$ 368 \quad \$ 368 \quad \$ 368$

$\$ 366 \quad \$ 366 \quad \$ 366$

$\$ 210 \quad \$ 210 \quad \$ 210$

50 $\$ 50 \quad \$ 340$

$\begin{array}{rrr}553 & 553 \quad \$ 53\end{array}$

$\$ 974$

$\$ 117 \$ 110 \quad \$ 103$

$\begin{array}{lll}\$ 700 & \$ 700 & \$ 100 \\ \$ 0 & \$ 0 & \$ 00\end{array}$

$\begin{array}{rrr}\$ 0 & \$ 0 & \$ 0 \\ 50 & \$ 0 & \$ 0 \\ 14,849 & \$ 19,621 & \$ 19,988\end{array}$

$\$ 33,126 \quad \$ 37,880 \quad \$ 43,267$ 
ANNEXE 9 Resultats d'exploitation previsionnels - Imagem

Revenus -ventes effectuees
Cout des marchandises vendues
Benefice brut ( 1 moins 2 )
Depenses

An 1

$1 \$ 237,603$

2

3

$\$ 7,029$

$\$ 230,574$
An 2

$\$ 255,639$

$\$ 7,350$

$\$ 248,289$
Salaires (bruts)
Sous-contrats
Avantages sociaux
Loyer
Location LT equipement
Taxes d'affaires, droits, permis
Assurance feu, vol, responsabilite
Location roulant
Frais de representation
Depenses de roulant
(essence, reparation, location)

Telephone

Fournitures de bureau

Honoraires de conseillers

Interets sur emprunt -court terme

Interet sur emprunt -long terme

Amortissement

Autres: demonstrateur

Autres: mauvaises creances, frais de collection et autres
6
$\$ 51,300$

$\$ 57,399$

$\$ 98,313$

$\$ 102,900$

$\$ 6,350$

$\$ 6,884$

$\$ 11,800$

$\$ 12,600$

$\$ 1,800$

$\$ 575$

$\$ 1,800$

$\$ 601$

$\$ 3,323$

$\$ 2,280$

$\$ 3,685$

$\$ 0$

$\$ 1,800$

$\$ 1,896$

$\$ 1,755$

$\$ 4,416$

$\$ 4,188$

$\$ 4,392$

$\$ 2,400$

$\$ 2,520$

$\$ 2,000$

$\$ 3,200$

$\$ 750$

$\$ 636$

$\$ 1,698$

$\$ 2,999$

$\$ 9,376$

$\$ 5,259$

$\$ 5,000$

$\$ 0$

$\$ 6,617$

$\$ 2,000$ 
ANNEXE 10 Bilans previsionnels - Imagem

\section{Actif}

Actif a court terme

-Encaisse

-Comptes a recevoir

- Frais payes d'avance

-stocks

-Autres (precisez)
(1)

(2)

(3)

(4)

(5)

Total
31 oct 87

$$
\begin{array}{r}
\$ 1,112 \\
\$ 27,469 \\
\$ 0 \\
\$ 1,780
\end{array}
$$

$\$ 30,361$
31 oct 88

$\$ 11,447$

$\$ 20,000$

$\$ 0$

$\$ 1,780$

$\$ 33,227$
31 oct 89

$\$ 43,267$

$\$ 25,000$

$\$ 0$

$\$ 1,780$

$\$ 70,047$

Immobilisations

- Terrain(s)

-Batisse(s)

-Materiel roulant (net)

-Mobilier

-Equipements

- Informatique
(7)

(8)

(9)

(10)

(11)

(12)

Total (13)

Total de l'actif (14)
$\$ 4,414$

$\$ 54,399$

$\$ 6,000$

$\$ 64,813$

$\$ 95,174$
$\$ 3,972$

$\$ 35,259$

$\$ 5,100$

$\$ 44,331$

$\$ 77,558$
$\$ 3,178$

$\$ 28,207$

$\$ 3,570$

$\$ 34,955$

$\$ 105,002$

Passif et avoir net

Passif a court terme

- Emprunt bancaire

- Comptes a payer

-Autres crediteurs

-Autres:

Total du passif

Avoir des actionnaires

- Capital-actions

- Subvention PECEC

- Pertes accumulees

Total de l'avoir net (26)

Total du passif et de l'avoir net
$\$ 44,125$ $\$ 53$

$\$ 44,178$

$\$ 23,489$

$\$ 70,000$

$(\$ 73,625)$

$\$ 19,864$

(27)

$\$ 23,489$

$\$ 70,000$

$(\$ 44,978)$

$\$ 48,511$

$\$ 95,174$

$\$ 77,558$
$\$ 18,000$

$\$ 9,200$

1847

$\$ 11,047$

$\$ 18,000$

$\$ 9.600$

$\$ 9,600$

$\$ 10,000$

$\$ 2,000$

$\$ 12,000$
$\$ 23,489$

$\$ 70,000$

$(\$ 10,087)$

$\$ 83,402$ 
IMAGEM PLAN OPÉRATIONNEL-CALENDRIER

.BUDGETS PRÉVISIONNELS

.BUDGET DES FRAIS

REPRÉSENTATION

- PARTICIPATION-ASSOCIA-

TIONS LOCALES

- PROGRAMMES DE SUBVENTION

.BUDGET DES DÉPENSES

D'OPÉRATION

- DÉF INITION DES TÂCHES/

COMPTABLE

. EMBAUCHE D'UN COMPTABLE

-pré-sélection

- sélection

-entrée en fonction

-intégration-formation

-VENTE D'ÉQUIPEMENTS

INUTILES

-RENCONTRE AVEC MILIEU

COMMUNICATIONS/BANQUE

DE RH

- INFORMATIONS ET CONTACTS

POUR SERVICES SPÉCIALISÉS

DE PRODUCITON

- PRÉPARATION D'UN DOSSIER CONCIS POUR LE BANQUIER

.CONTACTS AVEC LES PIGISTES RÉFÉRÉS

. PRÉPARATION D'UN DOSSIER

PAR PIGISTE

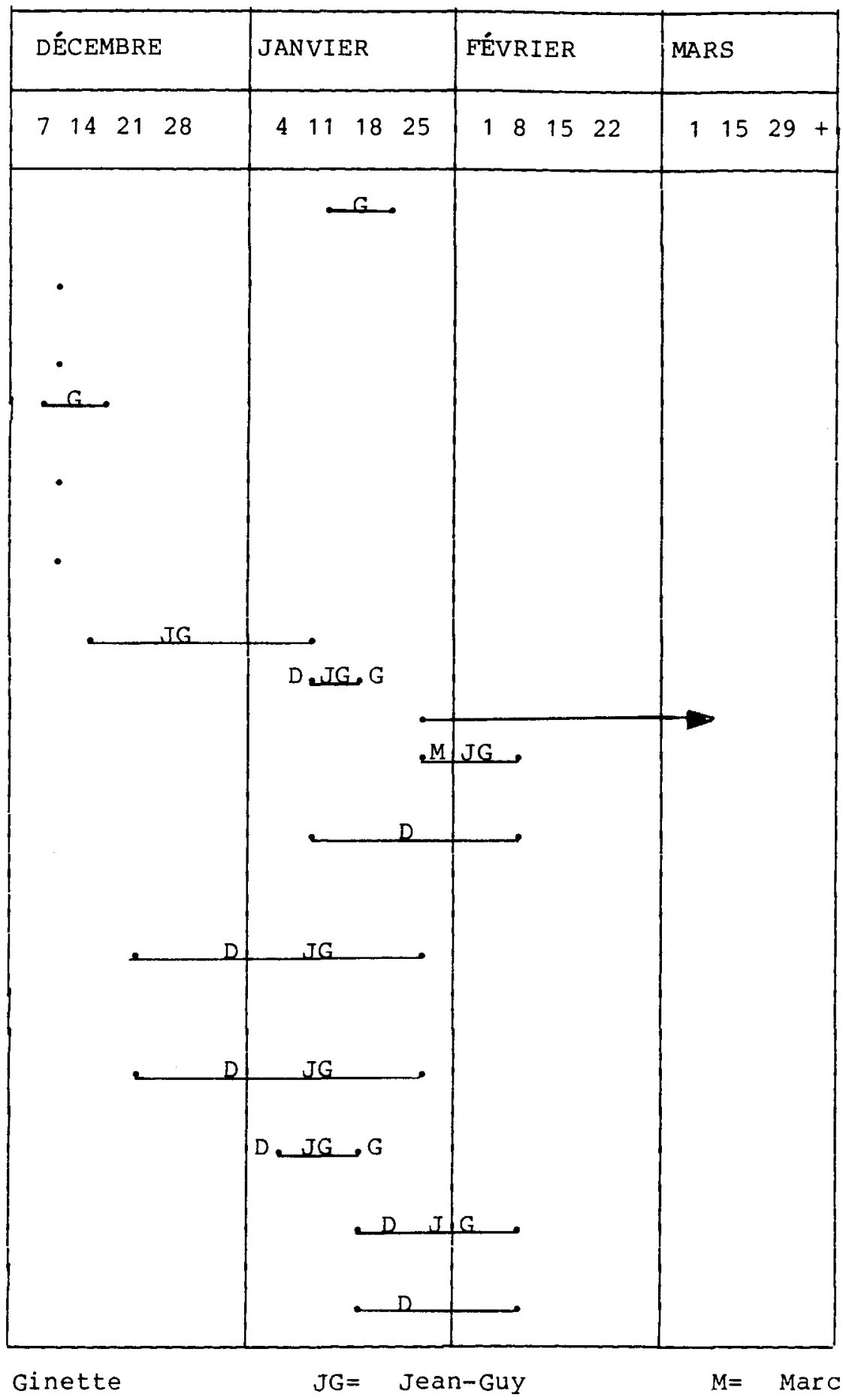

$D=$ Daniel

$\mathrm{G}=$ Ginette

$J G=J e a n-G u y$

$M=\quad \operatorname{Marc}$ 
IMAGEM PLAN OPÉRATIONNEL-CALENDRIER

. EMBAUCHE D'UN CONSULTANT EXTERNE/DEV. AUTO-CONSTRUCT ION

- PERCEPTION DES COMPTES A RECEVOIR INTENSIVE

-PRÉPARATION DE RAPPORTS VIDÉOTOUR EN RETARD

- EFFORTS ACCRUS DE RELATIONS PUBLIQUES

.FORMATION DU COMITÉ DE CONTRÔLE DE QUALITÉ

. SÉLECTION D'UNE RESSOURCE EXTERNE POUR L'ADMINISTRATION

- GRILLE DE SALAIRES OU FORFAITS ET AUTRES CONDITIONS D'EMPLOI/PIGISTES . CONTRAT D'EMBAUCHE TYPE/P IGISTES

-RENCONTRE BANQUIER POUR RESTRUCTURATION FINANCIÉRE

-GESTION DES PROJETS EFF ICACE

- RÔLE DU C.A.

-ORGANIGRAMME, RESPONSABILITÉS ET RÉPARTITION DES TÂCHES

- DOSSIER PRÉSENTATION D' IMAGEM

- DÉMO SPÉCIAL IMAGEM

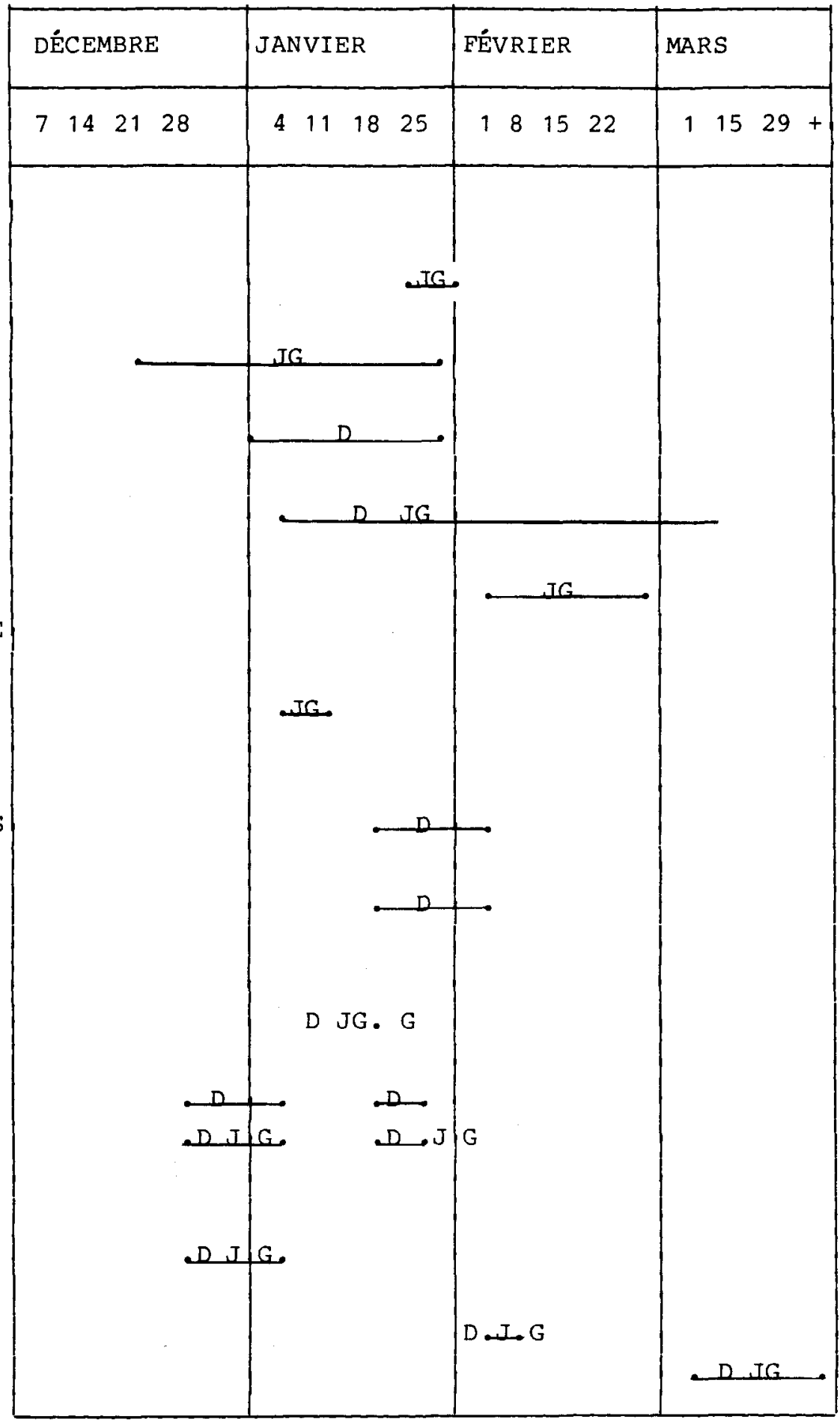


Acteur:

Décisions

administratives: celui (individu ou groupe) qui participe à une action et qui a des intérêts communs pour cette action.

Décisions

opérationnelles: dont le but est d'obtenir de l'exploitation courante, le maximum de profit.

Décisions

stratégiques:

qui intéressent les produits et les marchés que l'entreprise choisit, ses objectifs de développement et les orientations qu'elle se donne. 
Diagnostic

organisationnel: c'est une méthode d'évaluation qui consiste a faire un diagnostic poussé des principales méthodes de gestion en usage dans une entreprise de façon à vérifier si le processus administratif utilisé est adéquat et s'il comporte des lacunes sérieuses.

Management

stratégique:

il implique une approche de la direction de l'entreprise qui est: orientée vers le futur, systématique et créative. Son idée de base est que l'entreprise devrait continuellement scruter les développements actuels et futurs de son environnement et développer un concept stratégique qui guide et coordonne son évolution.

Planification

stratégique:

$$
\begin{aligned}
& \text { ce sont les procédures périodiques et } \\
& \text { formalisées relatives à l'analyse, à la } \\
& \text { conception des manoeuvres et à la mise en } \\
& \text { oeuvre de décisions stratégiques; qui } \\
& \text { permettent de rendre cohérent l'exercice. }
\end{aligned}
$$


Stratégie:

sys tème:

\section{Zones}

$d^{\prime}$ incertitude

désigne l'ensemble des critères de décision, choisi par le noyau, pour orienter, de façon déterminante et sur la durée, les activités et la configuration de I'entreprise.

c'est un ensemble d'éléments interdépendants, c'est-à-dire liés entre eux par des relations telles que, si l'une est modifiée, les autres le sont aussi et que, par conséquent, tout l'ensemble est transformé.

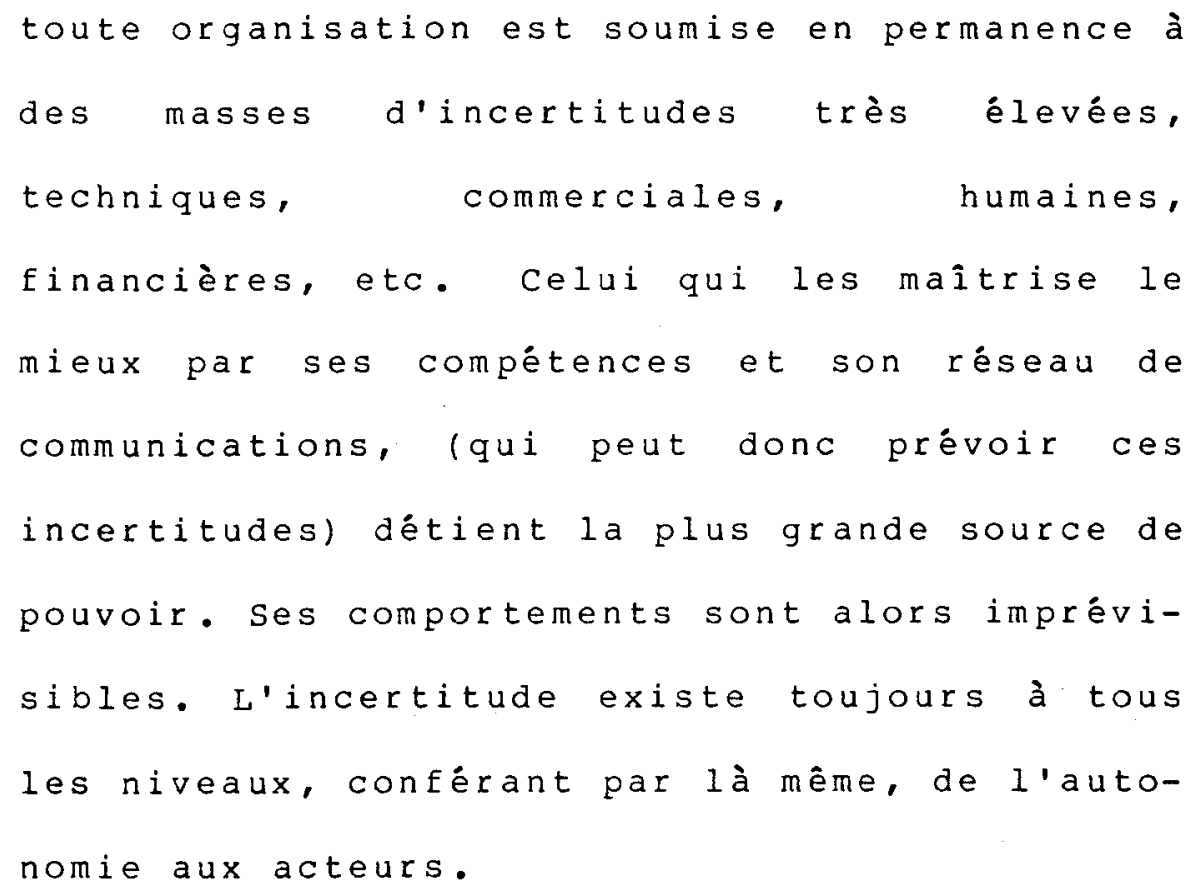


ANNEXE 13

CATÉGORIES D'ENTREPRISE

\begin{tabular}{|c|c|c|c|c|}
\hline \multirow[t]{2}{*}{$\begin{array}{l}\text { catégorie } \\
\text { d'entreprise }\end{array}$} & \multicolumn{2}{|c|}{ secteur fabrication } & \multicolumn{2}{|c|}{$\begin{array}{l}\text { secteur commerces } \\
\text { et services }\end{array}$} \\
\hline & $\begin{array}{l}\text { Nombre } \\
\text { d'employés }\end{array}$ & $\begin{array}{c}\text { chiffre } \\
\text { d'affaires } \\
\text { (en millier\$) }\end{array}$ & $\begin{array}{l}\text { Nombre } \\
\text { d'employés }\end{array}$ & $\begin{array}{c}\text { chiffre } \\
\text { d'affaires } \\
\text { (en millier\$) }\end{array}$ \\
\hline Artisanale & $0-4$ & $1-200$ & $1-3$ & $1-200 \$$ \\
\hline Petite & $5-49$ & 200 à 1500 & $4-10$ & 200 à 1000 \\
\hline Moyenne & $50-199$ & 1500 à 6000 & $11-30$ & 1000 à 5000 \\
\hline Grosse & $200-499$ & 6000 à 15000 & 31 et + & 5000 et + \\
\hline Très grosse & 500 et + & 15000 et + & - & $\longrightarrow$ \\
\hline
\end{tabular}

